\title{
SYNTHESIS AND CHARACTERIZATION OF THERANOSTIC AGENTS FOR PHOTOACOUSTIC IMAGING AND THERAPY
}

\author{
By \\ Yan Jie Wang \\ B.Sc. Ryerson University, 2012 \\ Toronto, Ontario

\begin{abstract}
A thesis
presented to Ryerson University

in partial fulfillment of the

requirements for the degree of

Master of Science

in the Program of

Biomedical Physics

Toronto, Ontario, Canada, 2015

(C) Yan Jie Wang, 2015
\end{abstract}




\section{Author's Declaration}

I hereby declare that I am the sole author of this thesis. This is a true copy of the thesis, including any required final revisions, as accepted by my examiners.

I authorize Ryerson University to lend this thesis to other institutions or individuals for the purpose of scholarly research.

I further authorize Ryerson University to reproduce this thesis by photocopying or by other means, in total or in part, at the request of other institutions or individuals for the purpose of scholarly research.

I understand that my thesis may be made electronically available to the public.

Yan Jie Wang 


\title{
Abstract
}

\section{Synthesis and Characterization of Theranostic Agents for Photoacoustic Imaging and Therapy}

\author{
Yan Jie Wang \\ M.Sc. Biomedical Physics, \\ Ryerson University, 2015.
}

In this work, phase-change contrast agents were developed for photoacoustic (PA) imaging and therapy. They consist of superheated liquid perfluorocarbon and gold nanoparticles capped by a Poly-(Lactide-co-Glycolic Acid) (PLGA) polymer shell. The phase transition from liquid to gas bubble can be remotely triggered by a laser source. In their liquid state, upon laser irradiation, these agents generated strong PA signals which were proportional to the laser fluence and particle sizes. The vaporization threshold decreased with increasing particle size, and was 850, 670 and $420 \mathrm{~mJ} / \mathrm{cm}^{2}$ for 2, 5, $10 \mu \mathrm{m}$-sized PLGA particles loaded with $35 \mathrm{~nm}$ GNPs, respectively. Cell culture studies, including passive uptake by the cancer cells and mechanical damage to the cancer cells caused by the vaporization inside the cells are also investigated. These agents show potential as photoacoustic imaging contrast and cancer therapy agents for clinical applications. 


\section{Acknowledgements}

I would like to express my sincere gratitude to my supervisor Dr. Michael Kolios who has given me this research opportunity and provided me his guidance and support during this work. His profound academic thought and integrity always inspire me. Under his supervision, I have learned many research skills which have laid a solid foundation in the path of my career as a researcher. I would also like to thank my supervisory committee, Dr. Carl Kumaradas and Dr. Devika Chithrani for their valuable ideas helped me to find the most effective way to achieve the goals of the project.

I would like to gratefully acknowledge our collaborators, Dr. Yang Sun, Dr. Chengcheng Niu, Professor Yuanyi Zheng, and Professor Zhigang Wang in Chongqing Medical University in

China. Their expertise on ultrasound molecular imaging helped me to achieve the design and synthesis of the contrast agents. Also, this work would not have been possible without the help from the senior researcher and the technical support provided by the lab; in particular, Dr. Eric Strohm for his expertise in acoustic microscope and his pioneer work on optical droplet vaporization, Elizabeth Berndl for her expertise in cell cultures, and Arthur Worthington for his assistance in other technical issues.

Finally, I would like to thank my family for all their love, support and believing in me. Especially I would like to express my gratitude to my husband Frank. Without his long-term encouraging, patience and support, this thesis work would not have been possible. 


\section{Table of Contents}

Author's Declaration ............................................................................................................................... ii

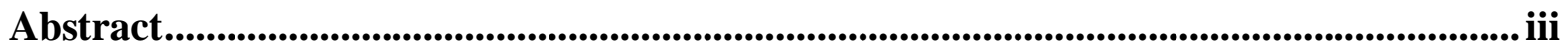

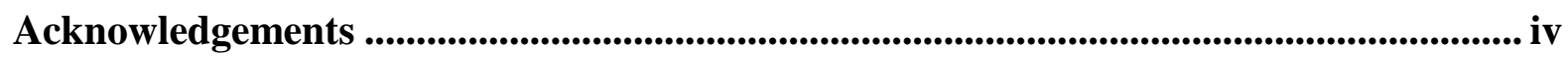

Table of Contents ........................................................................................................................... $\mathrm{v}$

List of Figures.................................................................................................................................... vii

Chapter 1 Introduction............................................................................................................................... 1

1.1 Thesis Overview .............................................................................................. 1

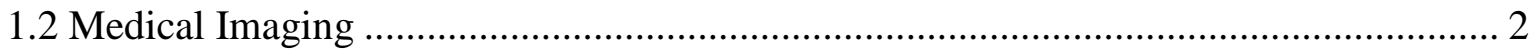

1.3 Photoacoustic Imaging ……………………………........................................ 5

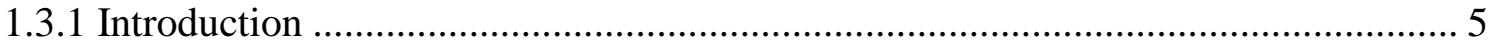

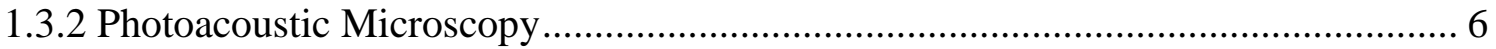

1.3.3 Initial Photoacoustic Pressure............................................................................ 7

1.4 Photoacoustic Contrast Agents............................................................................... 10

1.5 Phase Change Contrast Agents .................................................................................. 13

1.6 Mechanism of Photoacoustic Vaporization................................................................. 15

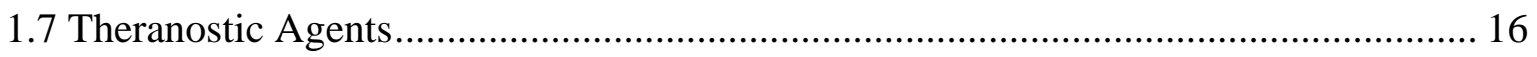

1.8 Hypothesis and Objectives ................................................................................. 18

Chapter 2 Design, Synthesis, and Characterization .................................................................... 20

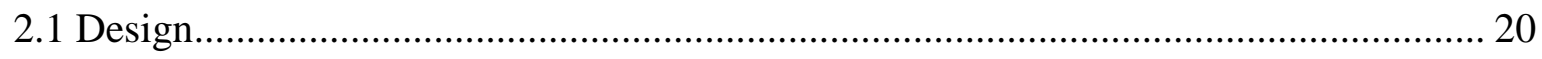

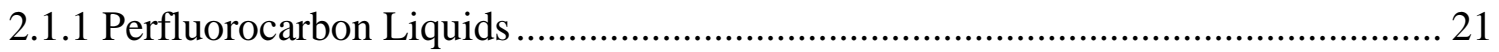

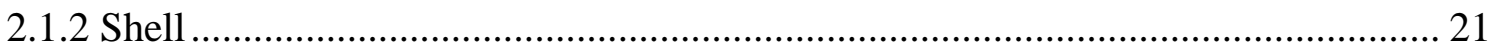

2.1.3 Plasmonic Nanoparticles ................................................................................... 23

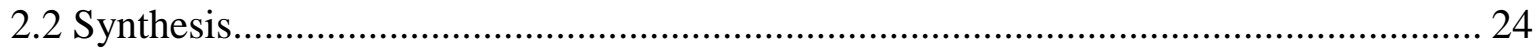

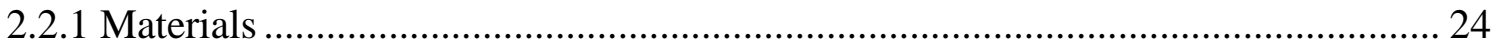


2.2.2 Silica-coated Gold Nanoparticle Preparation ................................................. 25

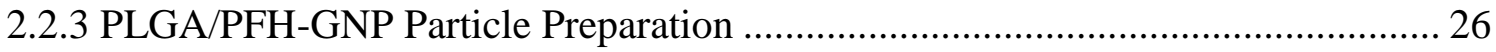

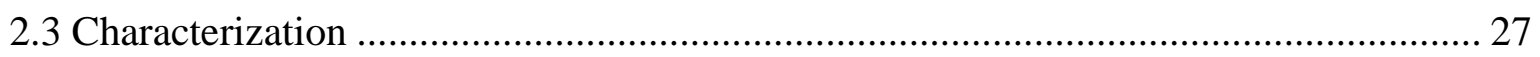

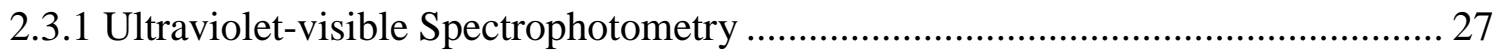

2.3.2 Transmission and Scanning Electron Microscopy .......................................... 28

2.3.3 Laser Diffraction Particle Size Analyzer.......................................................... 28

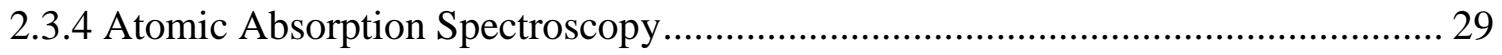

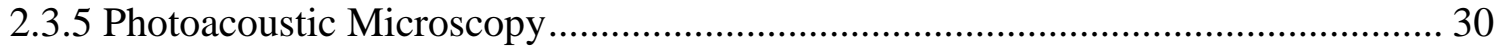

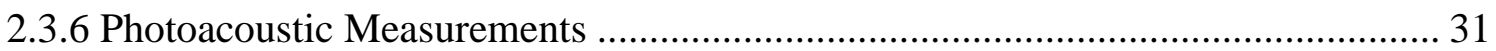

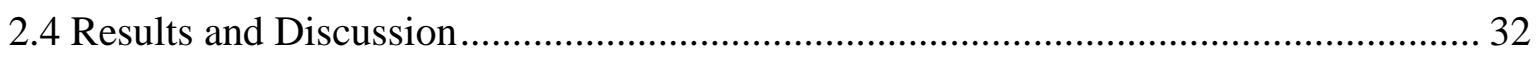

Chapter 3 In Vitro Study ................................................................................................................... 47

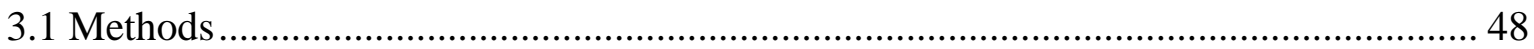

3.1.1 Passive Internalization of PLGA/PFH-GNP Particles by Cells .......................... 49

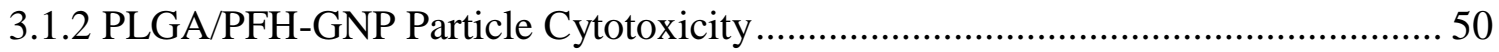

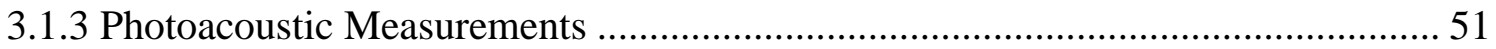

3.1.4 Mechanical Damage from Vaporization of PLGA Particles on Cells ................... 52

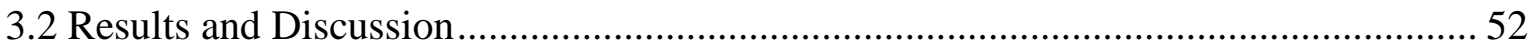

3.2.1 Passive Internalization of PLGA/PFH-GNP particles by Cells............................ 52

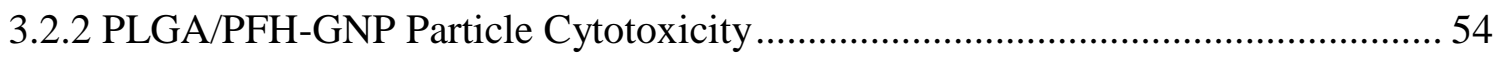

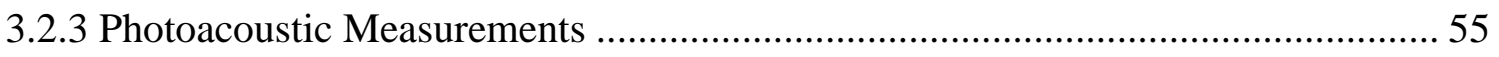

3.2.4 Mechanical Damage from Vaporization of PLGA Particles on Cells ................... 58

Chapter 4 Conclusion and Future Work .................................................................6 61

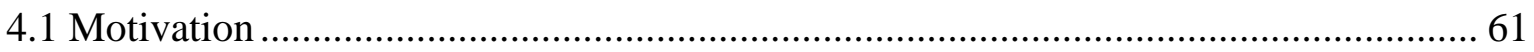

4.2 Phase Change Contrast Agent Synthesis and Characterization ................................ 61

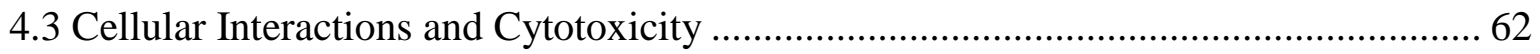

4.4 Contrast Enhancing Abilities of PLGA/PFH-GNPs ............................................ 62

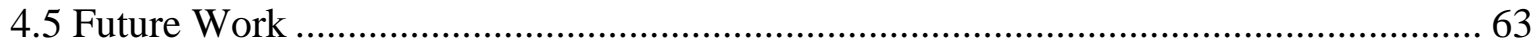

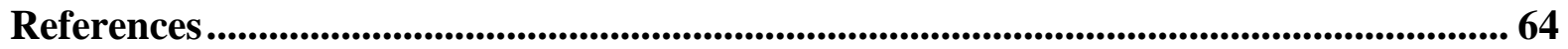




\section{List of Figures}

Fig. 1.1: Optical absorption spectra of major endogenous contrast agents in biological tissue at normal concentrations 10

Fig. 1.2: Optical absorption properties of plasmonic noble metal nanoparticles of different shapes and sizes

Fig. 1.3: Diagram of remote activation of a PFC droplet, providing PA signal via two mechanisms: thermal expansion and droplet vaporization

Fig. 1.4: Diagram of a multi-functional theranostic polymeric nanoparticle for simultaneous targeting, photo-imaging and drug delivery therapy 18

Fig. 2.1: The composition of a phase change contrast agent. .......................................... 20

Fig. 2.2: Synthesis process diagram of surface functionalized gold nanoparticles. .............. 25

Fig. 2.3: PLGA/PFH-GNP particle synthesis process diagram ........................................ 26

Fig. 2.4: A front view of the UV-Vis spectrophotometer used for the optical absorption measurement

Fig. 2.5: Photos of TEM and SEM device utilized for the structure and morphology characterization 28

Fig. 2.6: A photo of laser diffraction particle size analyzer.....

Fig. 2.7: A photo of the atomic absorption spectrometer that was used to measure the concentration of GNPs in solutions. 30

Fig. 2.8: Schematic demonstration of the photoacoustic microscope and the PA experimental setup.

Fig. 2.9: Experimental design for PA signal and vaporization threshold measurements ....... 32

Fig. 2.10: TEM images of silica-coated GNPs. .............................................................. 33

Fig. 2.11: The optical absorption spectra of three sizes of gold nanoparticls ....................... 33

Fig. 2.12: Gold nanoparticles in water and organic phase .............................................. 34

Fig. 2.13: SEM images of PLGA/PFH-GNP particles ................................................. 35 
Fig. 2.14: TEM images of single PLGA/PFH-GNP particles.

Fig. 2.15: The absorption spectra of $35 \mathrm{~nm}$ GNP solution and PLGA/PFH loaded with $35 \mathrm{~nm}$ GNP solution. 36

Fig. 2.16: The optical images and size distribution histogram of three types of PLGA/PFH-GNPs made from three sizes of GNPs 37

Fig. 2.17: Photoacoustic signals obtained from 2 and $10 \mu \mathrm{m}$ PLGA/PFH-GNPs. 38

Fig. 2.18: Optical and PA images of a $10 \mu \mathrm{m}$ PLGA/PFH-GNP particle and its PA signal obtained at the center of the particle 38

Fig. 2.19: The PA signal as function of the laser energy for three different particle sizes.... 39

Fig. 2.20: At the low energy level $\left(<200 \mathrm{~mJ} / \mathrm{cm}^{2}\right)$, the PA signal is linearly proportinal to the laser fluence. 40

Fig. 2.21: Optical images of PLGA/PFH-GNPs before and after vaporization. 42

Fig. 2.22: Photoacoustic signal amplitudes and vapoization threshold as functions of laser fluence and GNP sizes. 44

Fig. 3.1: A front view of a confocal laser scanning microscope. 50

Fig. 3.2: TEM images of a single PLGA/PFH-GNP particle internalized in a cytoplamic vesicle.

Fig. 3.3: Confocal fluorescence images of PLGA/PFH-GNP particles uptake by MCF7 and MDA cells. 54

Fig. 3.4: Cytotoxicity test resutls using the MTT assay. 55

Fig. 3.5: Optical, fluorescence and PA images of MDA cells loaded with DiI labeled PLGA/PFH-GNPs 56

Fig. 3.6: Pixel intensity profiles at the cross lines of fluorescence and PA images. 57

Fig. 3.7: A-scan PA signals obtained at the PLGA/PFH-GNP location and the background in the

PA image. 58

Fig. 3.8: Mechanical damage to the cells observed under optical and fluorescence microcope, (A) the cell slowly retracted after vaporization, (B) vaporization caused membrane disintegration evidenced by the PI fluorescence. 


\section{Chapter 1 - Introduction}

\subsection{Thesis Overview}

The purpose of a medical imaging modality is to detect pathology and to diagnose disease. Currently, the field of biomedical imaging is dominated by radiographic, magnetic resonance imaging, nuclear medicine imaging and ultrasound. Although these modalities are mature technologies, there exist short comings. They either utilizes ionizing radiation or are not cost effective, and cannot provide high resolution and functional information simultaneously [1][2].

Ultrasound (US) imaging provides images of tissue at relatively low cost. However, it cannot reliably distinguish between malignant and benign solid masses [3]. Optical microscopy imaging can provide extremely high resolution images, but have very low penetration depth due to the high scattering of light in the tissue [4]. Photoacoustic (PA) imaging utilizes acoustic waves generated from an object being illuminated by a laser source to deliver information to the surface of the tissue. It can generate high-contrast and high-resolution images of malignant tumors located deep within tissue [5]. The hemoglobin in the blood can serve as endogenous optical contrast. Tumors can be differentiated from normal tissues due to the increased angiogenesis inside the tumor [6]-[8]. However, for the early detection of malignant tumors, an exogenous contrast agent with high optical absorption is required to enhance the contrast.

Phase-change contrast agents have the advantages over liquid and gas bubbles contrast agents were first developed for ultrasound-based applications [9][10]. Some agents consist of superheated liquid perfluorocarbon (PFC) and plasmonic nanoparticles or dyes capped by a 
surfactant [11], lipid [12], protein [13] or polymer shell [14], and can be remotely triggered by a laser using a process called optical droplet vaporization (ODV) [15], to provide contrast for both PA and US imaging. In this project, a phase-change contrast agent is designed and synthesized, and its physical and optical properties are characterized. Its potential as cancer therapy agent is also investigated in vitro.

\subsection{Medical Imaging}

Medical imaging is the technique of creating images of the internal structure of a body which helps to identify abnormalities for clinical diagnosis and treatment of disease. It has become essential to medical care and treatment. By 2010, about 5 billion medical imaging studies had been conducted worldwide [16]. The common clinical medical imaging modalities include radiography (x-ray and computed tomography (CT)), nuclear imaging (positron emission tomography (PET)), single-photon emission computed tomography (SPECT), magnetic resonance imaging (MRI), ultrasound (US) imaging, and optical imaging (optical coherence tomography (OCT)). Each imaging modality has its advantages as well as its limitations. Which imaging modality is more appropriate depends on many factors including its efficacy in the detection of the pathology, costs, safety to the patients, and ease of access. The efficacy is mainly determined by the imaging contrast, spatial resolution, and image acquisition time (temporal resolution). Contrast in an image is the difference of an object compared to the background. It is based on different physical parameters in the patient (determined by the imaging modality used). Each modality also has limited spatial resolution which determines the ability to resolve fine detail in structures in the patient [1]. The wavelength of the energy used to probe the object is a fundamental limitation of the spatial resolution of an imaging modality. For example, optical 
microscope cannot resolve objects smaller than the wavelengths of visible light, about $500 \mathrm{~nm}$ [1].

\section{Radiographic Imaging}

In radiographic imaging, a homogeneous distribution of $\mathrm{x}$-rays with wavelengths ranging from 0.1 to $10 \mathrm{~nm}$ is modified due to different attenuation properties of tissues such as bone, soft tissue, and air inside the patient. The collected images are 2-D projections of the heterogeneous distribution of x-rays that emerges from the patient. 3-D images can be reconstructed based on 2D images using different computational methods [1]. X-rays and CT scans are the most commonly used medical imaging techniques due to their ability to rapidly scan large areas (10 seconds for a head scan), great penetration depth, and high spatial resolution (down to $0.01 \mathrm{~mm}$ ) [1]. However, this technique involves ionizing radiation which may incite the genesis of cancer at the radiation site. Contrast in x-ray images is dependent on the tissue density and the effective atomic number. It is also affected by the beam energy used. The better contrast images typically require higher exposures to the patient, which introduce more hazard to the patients [1].

\section{Nuclear Imaging}

Nuclear imaging uses radioactive decay of an exogenous contrast agent to construct images of the body. This typically requires the injection of a chemical containing a radioactive isotope into the patient. These compounds or tracers move selectively to different regions or organs within the body, emitting gamma rays with intensity proportional to the compound's local concentration [2]. Nuclear imaging is a form of functional imaging which can provide information about physiological condition, but has limited resolution (several $\mathrm{mm}$ ) and also 
exposes the patient to ionizing radiation. Contrast in nuclear images depends on the tissue's ability to concentrate the radioactive material which is dependent on the pharmacologic interaction of the agent with the body.

\section{Magnetic Resonance Imaging}

Magnetic resonance imaging (MRI) utilizes the nuclear magnetic resonance properties of the proton, which is very abundant in biologic tissues. When placed in a magnetic field, the proton having a magnetic moment, will preferentially absorb radio wave energy at the resonance frequency and release radio wave signals, which are detected and then reconstructed into highresolution (tens of micrometers) images of large volumes [2]. The MRI system uses the frequency (and phase) of the returning radio waves to determine the position of each signal in the patient. Images made using MRI demonstrate high sensitivity to anatomic variations [2]. Though MRI imaging does not use ionizing radiation, its scanning time is much longer than in CT imaging (tens of minutes for a head scan). In addition, the size and costs associated with operating an MRI device prohibit its widespread use. Contrast in MRI is related primarily to the proton density and to relaxation phenomena. Proton density is influenced by the mass density, thus differs among tissue types.

\section{Ultrasound Imaging}

Ultrasound imaging is a non-invasive, real-time, and cost-effective imaging modality. It primarily images tissue morphology through the reflection and scattering of high-frequency sound waves at tissue boundaries where the acoustic impedances of different tissues vary [16]. US imaging can discover the abnormality of anatomical boundaries and scattering behavior of 
tissues and can be used for pathology diagnosis [16]. US images are reconstructed from the reflected wave collected by transducers which traditionally use piezoelectric crystals to convert pressure waves into electric charge. Contrast in US imaging is largely determined by the acoustic properties of the tissue being imaged. The difference between the acoustic impedances of two adjacent tissues or other substances affects the amplitude of the returning US signal. The axial resolution of US images is directly related to the frequency of the transducers. High frequency transducers yield high axial resolution image, but with poor penetration depth. Diagnostic US is typically in the 3 to $15 \mathrm{MHz}$ range [17].

\subsection{Photoacoustic Imaging}

\subsubsection{Introduction}

Photoacoustic imaging is a new medical imaging modality based on the PA effect which is based on the generation of sound waves from the interaction of light with a medium. The PA effect was first discovered by Alexander Graham Bell in 1880, who found that sound can be generated from a selenium cell illuminated by sunlight [18]. The idea of utilizing the PA effect for biomedical imaging was first described in 1977 and it rapidly developed in the past two decades after advances in ultrasonic transducer, laser technologies and computer techniques [19]-[21].

Photoacoustics combines the advantages of optical and acoustical methods of high contrast and high resolution due to sensitive optical absorption and low acoustic scattering in soft tissue [22]. US imaging provides morphological data based on tissue composition boundaries. PA signals provide functional and molecular information due to optical absorption at the 
molecular level such as oxygenated and deoxygenated hemoglobin and melanin [23]-[25]. Furthermore, the incident optical irradiation wavelength can be selected to maximize absorption contrast between tissues with different absorption coefficient values [26]. In addition, multiple chromophores can be quantified simultaneously by varying the irradiating laser wavelength according to their optimal absorptions.

The spatial image resolution, as well as the maximum imaging depth, is limited by the ultrasonic frequency. As the ultrasonic center frequency and bandwidth increase, the spatial resolution improves at the expense of imaging depth, because ultrasonic attenuation increases with frequency [27]. Overall, because of its non-ionizing and non-invasive nature, ease of use and the fact that it is inexpensive, PA imaging has become the fastest growing new biomedical imaging modality, with clinical applications in development.

Photoacoustic signal can be generated through thermal expansion and vaporization mechanisms [28][29]. Vaporization is the conversion of liquid to the gaseous state which has higher PA signal generation efficiency than the thermal expansion process does [29]. But it requires relative higher amounts of optical energy comparing with thermal expansion. Thermal expansion is the least efficient of light-sound conversion mechanism; however it is the only biologically safe mechanism to date in biomedical applications, because tissue can only withstand low levels of laser irradiation without undergoing irreversible damage[30].

\subsubsection{Photoacoustic Microscopy}

In PA imaging, a short-pulsed laser beam irradiating the tissue is absorbed, and converted into heat. This heat is further converted to a pressure rise due to the thermal expansion of the absorbing structure. The initial pressure rise propagates as an ultrasonic wave, which is referred 
to as a PA wave. The PA wave is detected by an ultrasonic transducer as an electric signal. The signal is then amplified, digitized, and transferred to a computer to form an image. PA imaging has two major forms of implementation. One is based on a single element scanning-focused ultrasonic transducer. The other is based on unfocused array ultrasonic transducers. Photoacoustic microscopy (PAM) belongs to the first category [27].

In PAM, a focused ultrasound transducer records a time-resolved PA signal excited by a pulsed laser and emitted within the acoustic focal zone. By raster-scanning of the optical and acoustic foci, 2-D or 3-D images are formed [27]. The axial resolution of PAM is primarily determined by the frequency response of the ultrasonic transducer and the imaging depth. Its lateral resolution is determined by the product of the point spread functions of the dual foci. The detection sensitivity of PAM is affected mainly by the incident laser fluence, the optical absorption properties of tissues, and detection efficiency of the ultrasonic transducer [27].

\subsubsection{Initial Photoacoustic Pressure}

Upon short laser pulse excitation, the relationship between volume expansion fraction of the heated tissue $(d V / V)$, the pressure $(p)$ and temperature $(T)$ at position $r$ can be expressed as: [27]

$$
\frac{d V}{V}=-\kappa p(\mathrm{r})+\beta T(\mathbf{r})
$$


Here, $\kappa$ is the isothermal compressibility, $\beta$ is the thermal coefficient of volume expansion, $p$ and $T$ are the changes in pressure and temperature, respectively. The isothermal compressibility $\kappa$ can be expressed as [27]

$$
\kappa=\frac{C_{P}}{\rho v_{s}^{2} C_{V}} .
$$

Here, $\rho$ is the mass density, $v_{s}$ is the speed of sound, and $C_{P}$ and $C_{V}$ are the specific heat capacities at constant pressure and volume, respectively.

Thermal expansion occurs most efficiently when two conditions are met: thermal and acoustic (stress) confinement. Thermal confinement occurs when the laser pulse duration is shorter than thermal diffusion. Acoustic confinement occurs when the laser pulse duration is shorter than the acoustic wave propagation through the optical absorber [31]. For thermal expansion to take place, we assume that the laser pulse duration $t_{L}$ is less than the acoustic confinement time which is less than the thermal confinement time

$$
t_{L}<\frac{d_{c}}{v_{s}}<\frac{d_{c}^{2}}{4 \alpha_{\mathrm{th}}}
$$

where $d_{c}$ is the dimension of the optically absorbing target of interest, and $\alpha_{\mathrm{th}}$ is the thermal diffusivity.

Since the fractional volume expansion is negligible for such a short laser pulse, the local pressure rise $p_{0}$ immediately after the laser excitation can be derived from (1) [32] as

$$
p_{0}(\mathrm{r})=\frac{\beta T(\mathrm{r})}{\kappa} .
$$

If all absorbed optical energy converted into heat, and nonthermal relaxation such as fluorescence is negligible, the temperature rise generated by the short laser pulse is 


$$
T=\frac{A_{e}}{\rho C_{V}}
$$

where $A_{e}$ is the specific or volumetric optical absorption or optical energy deposition density (in joules per centimeter cubed). Combining (4) and (5), the initial pressure rise can be expressed as

$$
p_{0}=\frac{\beta}{\kappa \rho C_{V}} A_{e} \text {. }
$$

The Grueneisen parameter (dimensionless) is defined as

$$
\Gamma=\frac{\beta}{\kappa \rho C_{V}} .
$$

Hence, (6) becomes

$$
p_{0}=\Gamma A_{e}
$$

Since $A_{e}=\mu_{a} F$, where $\mu_{a}$ denotes the optical absorption coefficient and $F$ denotes the optical fluence (in joules per centimeter square), (8) becomes

$$
p_{0}=\Gamma \mu_{a} F
$$

Hence, the PA signal $\left(p_{0}\right)$ is proportional to the optical absorption coefficient. The absorption coefficient $\mu_{a}$ describes the probability of photon energy absorption in a medium per unit infinitesimal path length, and is heavily wavelength dependent. In a medium containing many absorbers of the same kind with number density of $N_{a}$ and absorption cross section of $\sigma_{a}$, the absorption coefficient $\mu_{a}$ is given by $\mu_{a}=N_{a} \sigma_{a}$. Usually using absorbers with high absorption cross section will increase the detection sensitivity. For example, plasmonic nanoparticles have larger absorption cross sections than most organic dye molecules [33]. 


\subsection{Photoacoustic Contrast Agents}

Photoacoustic imaging of tissues can be accomplished by measuring their optical absorption with the use of both endogenous and exogenous contrast agents. Endogenous contrast is nontoxic, usually abundant in tissue and do not perturb the original tissue microenvironment. The most commonly imaged endogenous contrast agents in PAM (grouped according to their absorption wavelength) include (1) in the ultraviolet (UV) (180-400 nm) and visible (VIS) (400$700 \mathrm{~nm}$ ) regions, DNA/RNA [34], cytochrome c [35], bilirubin [36], myoglobin [37], hemoglobin [26] and melanin [38]; and (2) in the near-infrared (NIR) region (700-1400 nm), lipid [39][40], water [41] and glucose [42]. Their absorption spectra are summarized in Fig.1.1. Among them, hemoglobin is commonly used for PA vascular imaging in the visible wavelength region due to its excellent contrast enhancement [43].

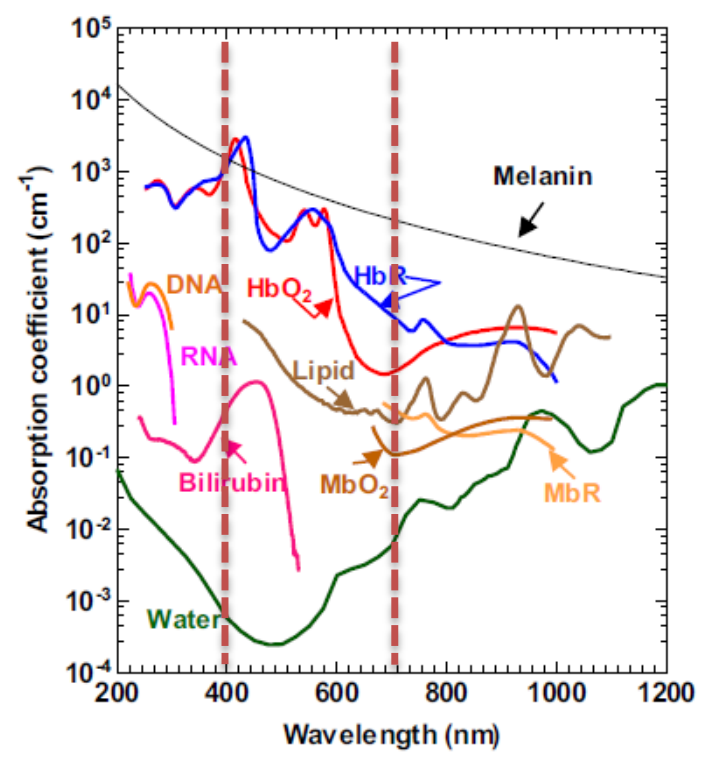

Fig. 1.1. Absorption spectra of major endogenous contrast agents in biological tissue at normal concentrations. Oxy-hemoglobin $\left(\mathrm{HbO}_{2}\right)$ and deoxy-hemoglobin ( $\left.\mathrm{HbR}\right)$ in blood; lipid, water, DNA and RNA in cell nuclei; melanin in medium human skin; myoglobin $(\mathrm{MbR})$ and oxy-myoglobin $\left(\mathrm{MbO}_{2}\right)$ in skeletal muscle, bilirubin in blood. The figure was adapted from [44]. 
Exogenous contrast agents not only have several-fold higher optical absorption coefficient value than exogenous contrasts, but also have other advantages over endogenous ones [4][44]. The chemical and optical properties of exogenous contrast agents can be specifically engineered for maximum detection sensitivity. In addition, exogenous contrast agents can be conjugated with targeting molecules (e.g., antibodies) to selectively bind to disease-specific cell surface receptors. Among them, organic dyes, such as indocyanine green [46][47] and Evan's blue [48], have distinct optical absorption properties, and can provide exogenous contrast. However, these dyes are prone to photobleaching, which limits their ability to produce sustained photoacoustic transients. Plasmonic gold nanoparticles have been extensively investigated for tumor targeting [49]-[51], cortical vasculature enhancement [46] and sentinel lymph node mapping [52]-[54] due to their inert chemical properties and excellent optical absorbing capabilities [4]. Plasmonic nanoparticles can also be used for therapeutic applications. The strong optical absorption of a contrast agent can, if using a continuous wave laser, produce a thermal heating effect by nanoparticles that could cause cell injury - an effect that can be used for photothermal therapy [55]-[57].

The superior optical absorption properties of plasmonic nanoparticles arise from their ability to undergo local surface plasmon resonance. When an electromagnetic wave interacts with a plasmonic nanoparticle, which has a wavelength similar to the size of the nanoparticle, the free surface electrons oscillate with the polarity of the inciting wave. The incident electric field alternates the polarization charges at the nanoparticle surface. The energy from the electromagnetic wave is absorbed, and released as heat, resulting in high attenuation (absorption) of the incident electromagnetic wave [58]. 
The synthesis of these nanoparticles can be tuned to produce a variety of different shapes, including nanospheres [59][60], nanoshells [61][62], nanorods [63][64], and nanocages [65][46] which will produce different optical absorption spectra. Also, the peak absorption wavelength may be varied by changing synthesis conditions to control the size and shape of the nanoparticle, as shown in Fig. 1.2. Multiple absorption peaks at different wavelengths arise from the different orientations in which the nanoparticle could interact with the incident wave. For example, gold nanorods exhibit two plasmon bands namely the longitudinal and transverse plasmon bands. The longitudinal plasmon band corresponds to light absorption and scattering along the long axis of the particle, and the transverse plasmon band corresponds to light absorption and scattering along the short axis of the particle. The optical properties of gold nanoparticles with resonance in the near infrared region around $1064 \mathrm{~nm}$ often allows for deep imaging within biological tissues due to the low optical absorption and scattering properties of tissue's constituents at this wavelength (what is known as a tissue "optical window") [66].

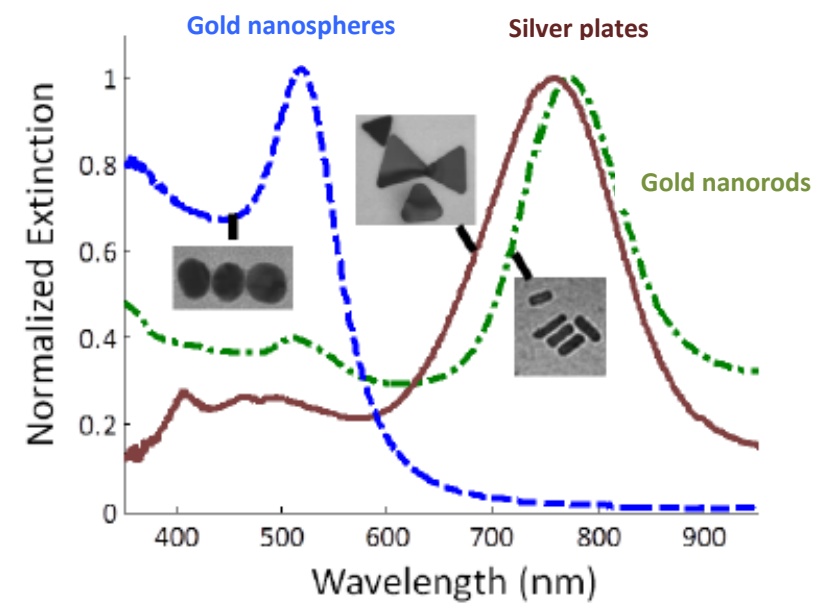

Fig. 1.2 Optical absorption properties of plasmonic noble metal nanoparticles of different shapes and sizes from left to right, gold nanospheres, silver plates, and gold nanorods. Adapted from [29]. 


\subsection{Phase-Change Contrast Agents}

Phase-change contrast agents, first developed for ultrasound-based applications [9], transform liquid emulsions into microbubble contrast agents that can have both diagnostic and therapeutic functions. When subjected to sufficient acoustic pressures delivered by an US pulse, perfluorocarbon (PFC) droplets undergo a volumetric expansion when the liquid cores change to gaseous states. This phenomenon, termed acoustic droplet vaporization (ADV) [67], has been proposed a number of in vitro and in vivo applications such as vascular occlusion [68], diagnostic imaging [69][70], ultrasound-mediated tissue ablation [71] and drug delivery [72][73]. Many in vitro studies have shown that the acoustic output necessary to induce vaporization increases as the droplet diameter decreases, the US frequency decreases, and the PFC boiling point increases [74][75]. Although a nanometer-scale droplet could provide a more efficacious vehicle for extravasation into tissue and activation at the site of action [68][73], applications involving relatively low frequencies may require pressures significantly higher than diagnostic US machines currently provide, increasing the potential for unwanted bioeffects [76].

Previous studies have shown that the vaporization of PFC droplets can be induced via optical irradiation with the facilitation of optical absorbers incorporated inside the droplets [13] [15] [77], termed optical droplet vaporization (ODV) [77]. When the laser fluence is below the vaporization threshold, and/or a laser wavelength is used that is not at the peak absorption wavelength of the droplet, the droplets remain in the liquid phase and can be used as a PA contrast agent. Increasing the laser energy, and/or using a laser wavelength at the peak absorption wavelength of the particle, induces droplet vaporization. After the vaporization, the resulting microbubbles can be used for contrast enhanced US imaging [78]. Bubble disruption can also be utilized for therapeutic purposes [78]. GNPs are commonly used as optical absorbing 
materials in these droplets due to their strong absorption cross section based on the surface plasmon resonance at visible and near-infrared regions [79].

Perfluorocarbon emulsions are commonly made from low-boiling point PFC liquids such as Dodecafluoropentane $\left(\mathrm{PFP}, 29^{\circ} \mathrm{C}\right)$ or perfluorohexane $\left(\mathrm{PFH}, 56^{\circ} \mathrm{C}\right)$ and stabilized by a surfactant [70][82], lipid [74][81], protein [67][75][13], or polymer [72][73][14] shell. When administered in vivo, liquid emulsions of high boiling-point PFCs are inert, non-toxic in small doses, and can have relative stability in circulation due to their high molecular weight, immiscibility in water, and low surface tension [82][83]. Depending on the choice of PFC, the emulsions may persist stably in vivo for hours to days [84], which is attractive for applications involving gradual accumulation at a target site and/or sustained release of drugs.

Droplets composed of a PFC liquid core encapsulated by a polymer shell has a lot of advantages: they are stable due to good mechanical strength than monomolecular layers of lipids or surfactants [85][86]; the elasticity of the shell can be controlled by adjusting the chemical compositions and the molecular weight of the polymer; they can also act as a good drug carrier or ligand for targeted imaging or targeted drug delivery [87]. With outstanding biocompatibility and biodegradability, Poly Lactide-co-Glycolide Acid (PLGA) slowly degrades in vivo into lactic and glycolic acid. These can further degrade into carbon dioxide and water via the tricarboxylic acid cycle [85]. So the PLGA is one of the most used synthetic biodegradable polymers in the drug delivery system [72][74], tissue engineering, and other biomedical fields, since it has been approved for clinical application [88]. 


\subsection{Mechanism of Photoacoustic Vaporization}

Thermal expansion is the predominant mechanism exploited for PA imaging, as the laser fluences used to produce acoustic transients are well within the ANSI standard limits [30]. However, thermal expansion is one of the least efficient energy conversions between light and sound, with the conversion efficiency $(\eta)$ around $10^{-4} \%$ [89]. Vaporization, the conversion of liquid to the gas phase is much more efficient than thermal expansion at converting light to acoustic transients, with $\eta$ close to $1 \%$ [28]. If vaporization could be utilized in biomedical imaging applications, this would allow for deeper tissue imaging, and help to minimize the concentration of contrast agents used. The conversion of liquids to gas bubbles using pulsed laser irradiation has been studied in depth [90][91]. For a single bubble, with a smooth surface, that is perfectly spherical and unbound, the pressure $\left(p_{1}\right)$ emitted by the growing vapor volume can be defined as [92]

$$
p_{1}(r, t)-p_{0}=\frac{\rho_{1}}{4 \pi r} \frac{d^{2} V\left(t-\frac{r}{c}\right)}{d t^{2}}
$$

where $p_{0}$ is the ambient liquid pressure surrounding the source, $r$ is the radius from the vaporization center, $t$ is the time, $\rho$ is the liquid density, $V$ is the velocity of the bubble expansion, and $c$ is the speed of sound in the liquid. The equation shows that the emitted pressure is dependent on the initial volume of the gas, which is limited by the droplet size.

During the vaporization process, upon a pulsed laser irradiation, the encapsulated plasmonic nanoparticles absorb electromagnetic energy from the laser, and cause the local temperature increase well over the required vaporization temperature of PFC (steps 1 through 2 
in Fig. 1.3). Meanwhile, plasmonic nanoparticles generate a high frequency pressure wave at their surface via thermal expansion. The heat and pressure together provide the conditions for nucleation of a liquid-to-gas phase transition of PFC. The liquid droplet undergoes a vaporization which overcomes the surface tension of the shell material (step 3 in Fig. 1.3). The PA transient produced via vaporization is substantially larger than the transients produced from the plasmonic nanoparticles which undergo thermal expansion. Furthermore, the resulting gaseous phase also produces an increased acoustic impedance mismatch between gas bubbles and the surrounding medium, providing optically triggered ultrasonic contrast enhancement [29].

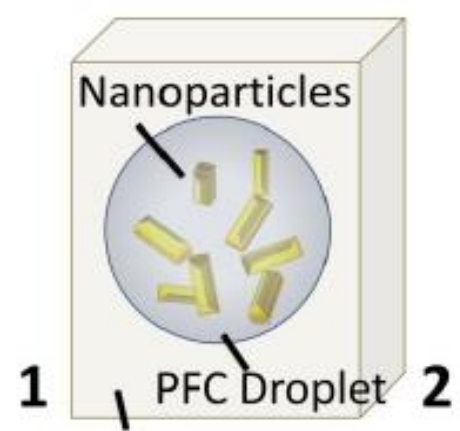

Tissue

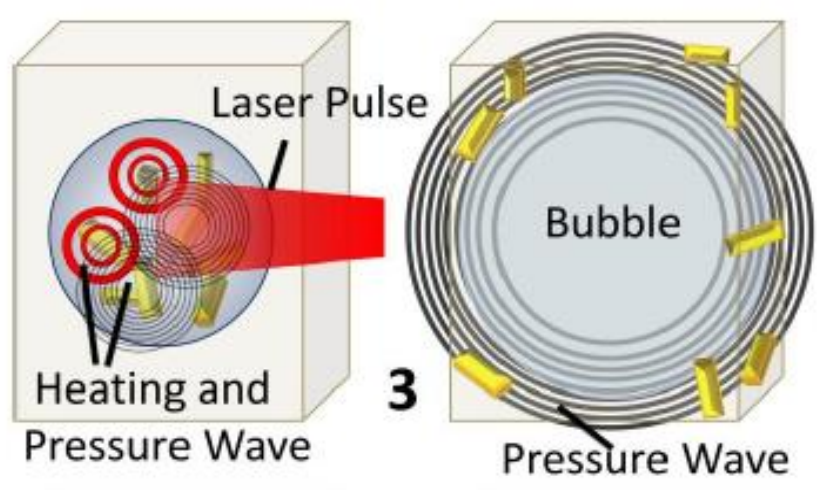

Fig. 1.3 Step-by-step diagram of remote activation of a PFC droplet, providing PA signal via two mechanisms: droplet vaporization and thermal expansion caused by plasmonic nanoparticles. The resulting gas microbubble of PFC provides ultrasound contrast due to increased acoustic impedance mismatch. Adapted from [29].

\subsection{Theranostic Agents}

With the increasing incidence of fatal diseases like cancer, there is a growing need for greater efficacy of therapeutic agents and precise methods of diagnosis. Theranostic agents have 
been recently developed to assist simultaneously deliver imaging and therapeutic agents for combined diagnosis and therapy. These multi-functional agents can be used for disease diagnosis and imaging applications via contrast agents with modalities such as OCT, MRI and fluorescence imaging. Further, therapy can be carried out using light-activated hyperthermia and by modulating the drug release rates based on the application and the polymer chosen [93].

Recent research in photo-based nanomedicine has gained prominence in the treatment of cancer and other diseases due to spatially and temporally controllable drug release, localized therapy and minimally invasive nature of treatment [93]. Several biomaterials and their complexes are being researched for encapsulation of light-responsive agents for photo-triggered treatment and imaging [94]. In the recent study by Bogart et al. [95], dextran polymer-coated iron oxide NPs were used for photothermal microscopy. Their results suggest increased sensitivity and resolution in imaging, indicating promising imaging abilities for in vivo study. PLGA polymer has also been highly utilized in different nanocarrier systems. Recently, Cheng et al. [96] prepared $\mathrm{Fe}_{3} \mathrm{O}_{4}$ and QD-conjugated taxol-loaded PLGA nanoparticles (NPs) onto which poly(styrenesulfonate)-coated GNRs were attached. These multi-functional NPs could be used for chemotherapy and NIR-based photothermal ablation of cancer cells. The results of in vitro and in vivo studies indicate that the combination of chemotherapy and photothermal therapies mediated by these NPs provides a more effective treatment against cancer compared to either of these treatments alone. These studies demonstrate the potential of use of different phototriggered materials in combinational therapy approaches in future cancer treatment. 


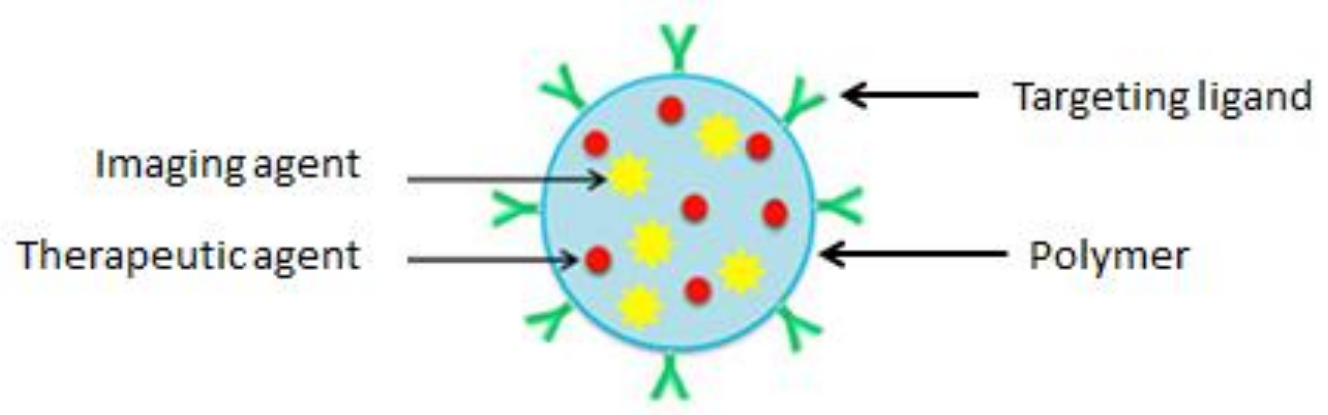

Fig 1.4. Multi-functional theranostic polymeric NPs for simultaneous targeting, photo-imaging and drug delivery/therapy. Adapted from [97].

Many new types of polymers and systems synthesized could be used for multiple drug release and photothermal treatments as well as multiple imaging strategies [94][98]. Fig.1.4 shows multifunctional nanocomposites combine therapeutic, diagnostic, and sensing modalities in a single nanostructure [97]. At present, multifunctional GNP-based nanocomposites [99] offer new theranostic modalities by combining the unique optical properties of plasmonic GNPs with the properties of mesoporous silica functionalized with an appropriate photosensitizer and molecular probes [100]. Nanoparticles made of noble metals such as gold and silver are prominent due to their highly desirable and tunable optical properties. These properties make them invaluable for various biological sensing, imaging and therapeutic applications.

\subsection{Hypothesis and Objective}

The hypothesis of the study is that optical-triggered phase-change contrast agents can be developed and utilized as dual purpose imaging and therapeutic agents. In order to test the hypothesis, the overall goal of this project is to design, synthesize, and test a phase-change contrast agent for PA imaging and cancer therapy. In order to accomplish this goal, several steps 
were taken to ensure its feasibility. Firstly, design, synthesis, and characterization of the contrast agent were undertaken. Secondly, the agent was tested in phantom experiments to study the PA contrast enhancement and characteristics in terms of the physical and optical properties of these particles. Thirdly, in order to achieve maximum PA detection sensitivity in vitro, the optimal light exposure parameters were studied. Finally, the feasibility of particle vaporization inside cancer cells and damage to the cell caused by the vaporization was investigated.

This thesis is organized in the following manner. Chapter 1 reviews background information on basic medical imaging modalities and more detailed information on PA imaging, and its current contrast agents. In Chapter 2, the phase-change contrast agent, GNPs and PFC liquid loaded PLGA particles, are designed, synthesized and characterized. The results from in vitro study are described and discussed in Chapter 3. Conclusion and future work are presented in Chapter 4. 


\section{Chapter 2 - Design, Synthesis, and}

\section{Characterization}

The overall objective of this research project is to design and synthesize contrast agents for PA imaging and cancer therapy. The design, synthesis, and physical characterization of the contrast agents are explained in detail in this chapter. The choice of each material as a design component is discussed. Furthermore, the synthesis procedure is outlined here. Finally, the physical characterization results are shown.

\subsection{Design}

The agent used in our design comprises a PFC liquid in the core stabilized by a PLGA polymer shell, and silica-coated gold nanospheres in the core for acting as PA contrast and as a "fuse" to vaporize the PFC. Each component as illustrated in Fig. 2.1 will be discussed in the following section, including their usage in history in biomedical applications and specific design criteria met by their inclusion.

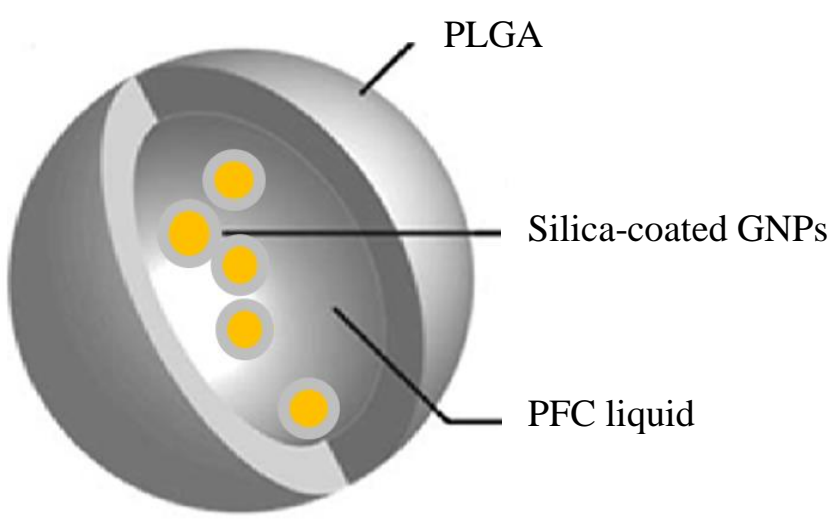

Fig. 2.1 The composition of a phase change contrast agent. 


\subsubsection{Perfluorocarbon Liquids}

Perfluorocarbon (PFC) gas microbubble has a long history as an exogenous contrast agent for ultrasound contrast enhancement, and has long been used in diagnostic ultrasound imaging as highly sensitive, cost effective, and biocompatible contrast agents [101]. Due to their highly scattering acoustic properties and nonlinear interactions with incident ultrasound, microbubbles are used in many clinical applications. However, gas diffusion and biological clearance significantly limit their circulation time and their micron-size inhibits their extravasation out of the vascular system [102]. Phase change, ultrasound activated, contrast agents were introduced to increase circulation time and stability. In our design we use an optical activation strategy to induce the PFC phase transition from liquid to gas.

The choice of which specific PFC would be used in this research was determined by examining several factors including historical usage, boiling temperature, ease of handling, and cost. In order to stay in the liquid phase in the body prior to any triggered vaporization event, and to avoid any special requirements for handling the PFC during the synthesis process, the PFC boiling temperature was chosen to be above body temperature $\left(37^{\circ} \mathrm{C}\right)$. For these reasons, perfluorohexane $(\mathrm{PFH})$ (boiling point is around $56{ }^{\circ} \mathrm{C}$ ) was chosen as the $\mathrm{PFC}$ to be used in this research.

\subsubsection{Shell}

The difference in pressure between the inside and outside of a droplet, named Laplace pressure can be represented with the following equation derived from the Young-Laplace equation for a sphere: [103] 


$$
\Delta P=P_{\text {inside }}-P_{\text {outside }}=\frac{2 \gamma}{R}
$$

where $\Delta P$ is the Laplace pressure, $P_{\text {inside }}$ is the pressure on the inside of the droplet, $P_{\text {outside }}$ is the pressure of the environment on the outside of the droplet, $\gamma$ is the surface tension on the droplet, and $R$ is the droplet radius. This equation indicates that the smaller the radius of the particle, the smaller the surface tension and the higher the Laplace pressure becomes. Stabilization is usually facilitated with a surfactant, capping agent, or shell. Shell molecules limit surface tension by decreasing the interfacial resistance between the droplet and the surrounding aqueous environment [104]. Furthermore, this shell can prevent vaporization of the droplet at environmental temperatures higher than the PFC boiling point and allows the droplet to become super-heated without vaporizing.

Three types of shells are typically used for US contrast agents including lipid, polymer, and protein shells. Lipid shells primarily comprise of phospholipid bilayers and cholesterols to aid in stability. They are easy to synthesize, bioinert [105], and are easily functionalized with targeting moieties and therapeutic cargoes [106]. However lipid shells are difficult to degrade in the body and vaporization more difficult due to the shell elasticity [107]. Protein shells are traditionally made with bovine serum albumin (BSA) [67] which can withstand denaturing at temperature up to $69^{\circ} \mathrm{C}$ [108] and pHs between 4.0 and 8.5 [109]. Furthermore, albumin is known to have passive targeting effects toward the surface of cells. BSA shells are also easily functionalized with biotin/avidin bridges connected to monoclonal antibodies or other small molecule targeting strategies. However, using bovine serum albumin has been shown at times to cause an immune response in humans [29]. Finally, polymer shells typically made of a biodegradable and biocompatible material like PLGA [85][110]-[112] is a FDA approved material for clinical applications. These shells are the most resistant to pressure waves, as 
compared to lipid or protein shells [86] which can leave sufficient time for the agents to accumulate at the target sites. In addition, polymer shells have the ability to facilitate targeting and encapsulate therapeutic cargoes [85][86][113]. The shell thickness can also be controlled by changing the ingredient ratio during the synthesis. For these reasons, PLGA was chosen as the shell for the design.

\subsubsection{Plasmonic Nanoparticles}

Exogenous contrast agents can produce PA contrast which is several orders of magnitude higher than the intrinsic contrast alone if used in sufficient quantities. Plasmonic noble metal nanoparticles, primary made from gold [59][62][114]-[117] and silver [65][118], are the most frequently used exogenous contrast agents. The localized surface plasmon resonance may be varied by changing synthesis conditions and finely tuning the absorption characteristics. The synthesis of these nanoparticles is easily controllable to produce a variety of shapes, including, but not limited to, nanospheres [119]-[121], nanoshells [62][122], nanorods [123]-[126], and nanocages [127][46].

Gold nanospheres are chosen as optical absorbers due to their small size (approximately 10-100 nm) compared to a PFC droplet (approximately $200-1000 \mathrm{~nm}$ ), allowing many of them to be encapsulated per droplet. The peak absorption wavelength of gold nanospheres is around $530 \mathrm{~nm}$ which is suitable for laser equipment generally available. Using silica-coating on the gold nanospheres helps miscibilizing gold nanoparticles in PFC liquids. By modification of the surface properties of silica using ligand exchange protocol facilitates PFC dispersion. In addition, silica has been reported to be good bio-compatible material. Silica-coating can provide enhanced colloidal stability functional groups for bioconjugation. Silica is a stabilizer which not only 
prevents particle coalescence but also is chemically inert and optically transparent [128]. Furthermore, the dielectric shell of silica has a strong influence on the amplitude of the generated photoacoustic signal. Silica-coated gold nanorods of the same optical density are capable of producing about 3-fold higher photoacoustic signals than gold nanorods without silica coating [83]. The enhancement is caused by the reduction of the gold interfacial thermal resistance with the solvent due to the silica coating [82].

\subsection{Synthesis}

PFC liquids are hydrophobic and lipophobic. In order to incorporate the gold nanoparticles within the PFC droplets, several steps are required. First step is to coat GNPs with a thin layer of silica followed by fluorination. After GNPs are miscibilized into PFCs, the GNPsPFC solution can be emulsified using solvent evaporation method to form different sizes of GNPs-incorporated PFC droplets.

\subsubsection{Materials}

All chemicals were used without additional purification and were at least at ACS grade. Hydroauric acid $\left(\mathrm{HAuCl}_{4}\right)$ (99\%), Trisodium Citrate $\left(\mathrm{Na}_{3} \mathrm{C}_{6} \mathrm{H}_{5} \mathrm{O}_{7}\right)$ (99\%), Tetraethyl orthosilicate (TEOS), 1H,1H,2H,2H-perfluorodecyltriethoxysilane, $\mathrm{CH}_{2} \mathrm{Cl}_{2}$, PLGA and $\mathrm{PFH}$ were purchased from Sigma Aldrich Inc. Acetone, isopropanol, methanol, ammonia was purchased from Fisher Scientific Inc. Deionized water (Millipore Milli-Q grade) with resistivity of $18.2 \mathrm{M} \Omega$ was used in all experiments. 


\subsubsection{Silica-coated Gold Nanoparticle Preparation}

The synthesis of citrate-stabilized gold nanospheres based on the single-phase aqueous reduction of tetracholoauric acid by sodium citrate, initially was developed by Turkevich et al. in 1951 [129] and further refined by Frens et al. [130], still remains the most commonly employed aqueous method. The resulting particles were coated with negatively charged citrate ions and hence are well suspended in $\mathrm{H}_{2} \mathrm{O}$ [131]. To achieve the silica-coating on GNPs, GNP solution was mixed with isopropanol, under vigorous shaking, ammonia was added to the mixed solution, followed by the addition of TEOS in isopropanol and pure silica-coated GNPs $\left(\mathrm{Au} @ \mathrm{SiO}_{2}\right)$ particles were formed [132]. The next, $\mathrm{Au} @ \mathrm{SiO}_{2}$ can be suspended into the PFH phase using ligand exchange with $1 \mathrm{H}, 1 \mathrm{H}, 2 \mathrm{H}, 2 \mathrm{H}$ perfluorooctanethiol. Then the surface modified $\mathrm{Au} @ \mathrm{SiO}_{2}$ were dispersed in PFH by sonication for several minutes, which resulted in a clear solution [133].

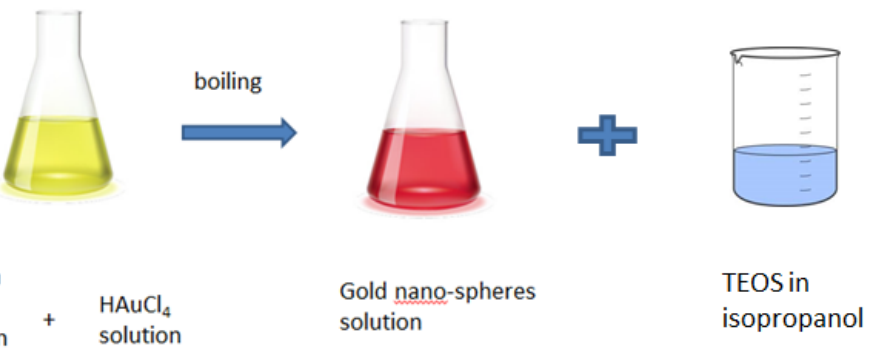

(Turkevich et al. in 1951, refined by Frens et al.)

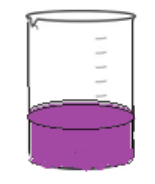

Miscibilization of the silica-coated NPs into PFCs

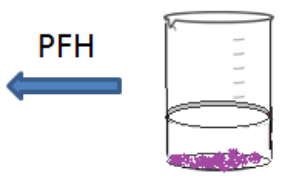

fluorinated silica coated NPs

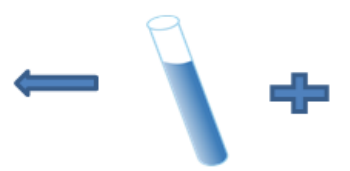

$1 \mathrm{H}, 1 \mathrm{H}, 2 \mathrm{H}, 2 \mathrm{H}-$ perfluorodecyl triethoxysilane

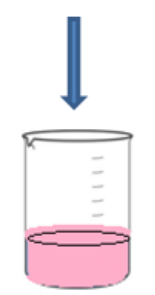

Silica coated gold nano-spheres solution

Fig. 2.2 Synthesis process of surface functionalized GNPs. The final products are fluorinated $\mathrm{Au} @ \mathrm{SiO}_{2}$ dispersed in PFC liquids. 


\subsubsection{PLGA/PFH-GNP Particle Preparation}

PLGA particles containing PFH and silica coated GNPs with and without dialkylcarbocyanine fluorophores (DiI) dye were prepared using a double emulsion (water/oil/water) solvent evaporation process [134][78]. Briefly GNPs-PFH solution (0.5 mL) was added to PLGA $(50 \mathrm{mg})$ in dichloromethane $(1 \mathrm{~mL})$. The mixture was emulsified in an ice water bath for 45 seconds, with 2-second-on, 1-second-off, $10 \mathrm{~W}$ pulses, using a digital sonifier (BRANSO, USA) equipped with a microtip. Then the emulsion was homogenized with polyvinyl alcohol solution. The final emulsion was mixed with $2 \%$ isopropanol solution and stirred for 2 hours to vaporize organic solvents and washed several times by centrifugation. The final product of PLGA particles loaded with PFH and GNPs were collected and stored at $4{ }^{\circ} \mathrm{C}$ for future usage. The overall procedure is shown schematically below (Fig. 2.3).

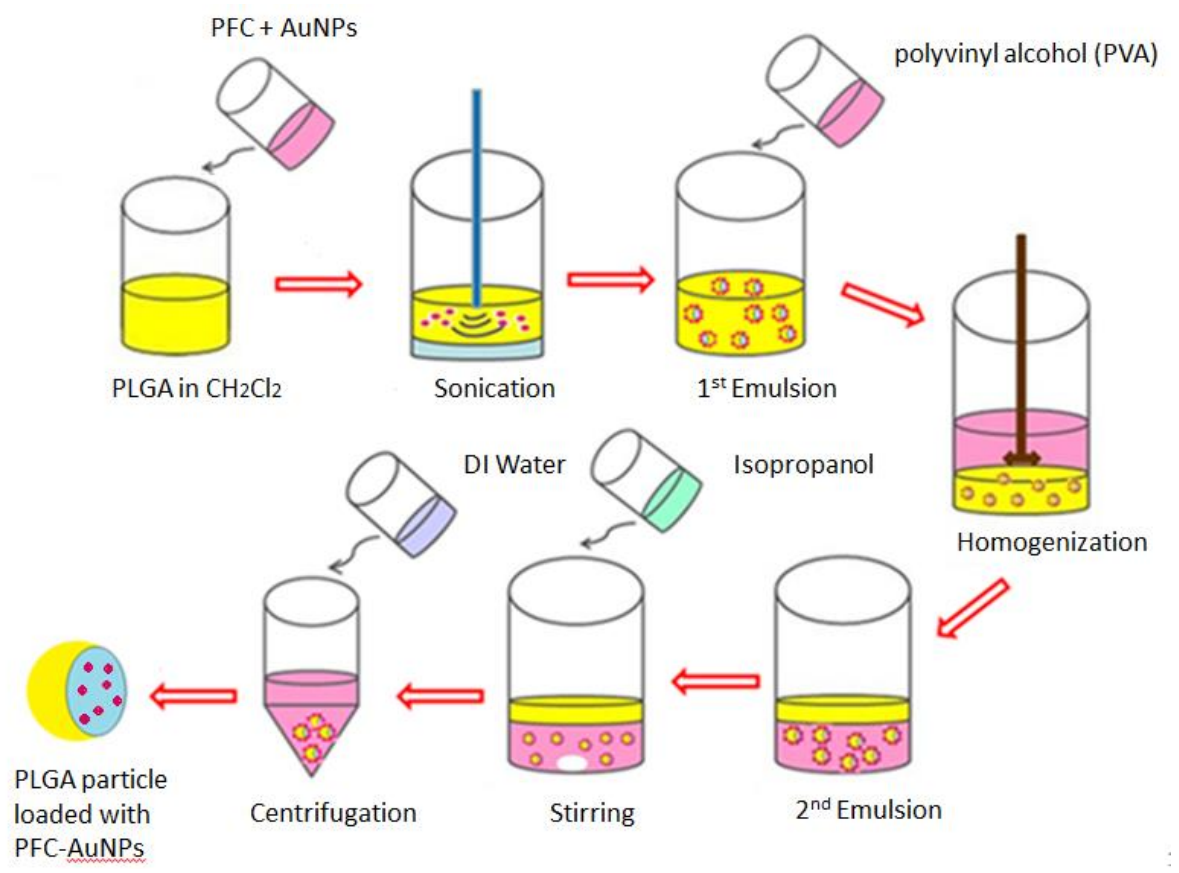

Fig. 2.3 PLGA/PFC-GNP particles are synthesized using w/o/w double emulsion method. The final products are PLGA/PFC-GNP particles with GNPs and a PFC liquid in the core stabilized by PLGA shell. 


\subsection{Characterization}

Several methods and tools were used to characterize the optical and physical properties of GNPs and PLGA/PFH-GNP particles. First ultraviolet to near infrared spectrophotometry (UVNIR) was used to monitor optical absorption properties. Next, transmission electron microscopy (TEM) and scanning electron microscopy (SEM) were used to qualify nanoparticle loading, location and morphology of PLGA/PFH-GNP particles. Additionally, dynamic light scattering was used to determine the size distribution of PLGA/PFH-GNP particles. Finally, an atomic absorption spectroscopy (AAS) technique was used to quantify the GNPs upload efficiency. The combination of these characterization techniques yielded important information about the optical and physical properties of PLGA/PFH-GNP particles.

\subsubsection{Ultraviolet-Near Infrared Spectrophotometry}

The optical absorption of different sample solutions was measured using a UV-NIR spectrophotometry (UV-3600, Shimadzu, Japan) (Fig. 2.4). PLGA/PFH-GNP particles were synthesized as described above. Their optical density was measured in a $1 \mathrm{~cm}$ plastic cuvette.

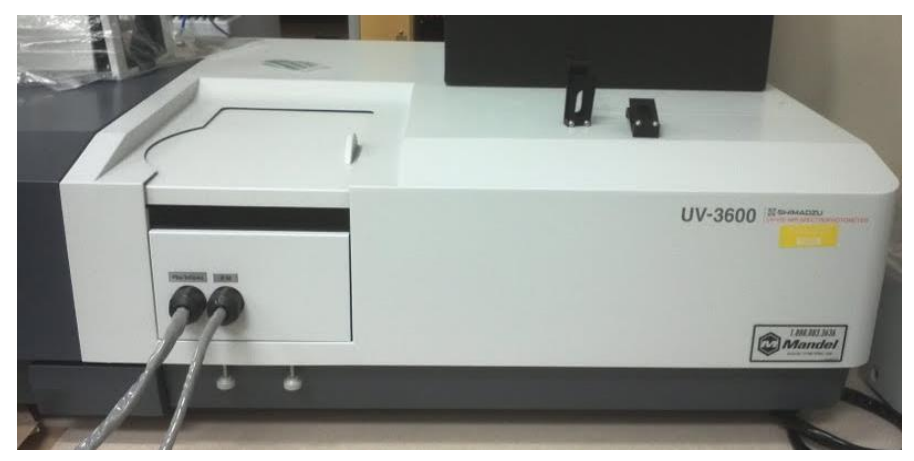

Fig. 2.4 A front view of the UV-NIR spectrophotometer used for the optical absorption measurement. 


\subsubsection{Transmission and Scanning Electron Microscopy}

Both transmission electron microscopy (FEI Tecnai 20, Philip, Japan) and scanning electron microscopy (FEI XL30 ESEM, Philip, Japan) (Fig. 2.5) were used to image the PLGA/PFH-GNP particles. For the TEM measurement, a $10 \mu \mathrm{L}$ diluted solution of PLGA/PFHGNP particles was placed on a carbon-coated copper grid. After the sample was dried, the images were taken at $200 \mathrm{keV}$ energy level. For the SEM measurement, the sample was coated with a thin layer of gold film and scanned at $20 \mathrm{keV}$ energy level. The contrast agent characterization allowed for study of both physical and optical characteristics.
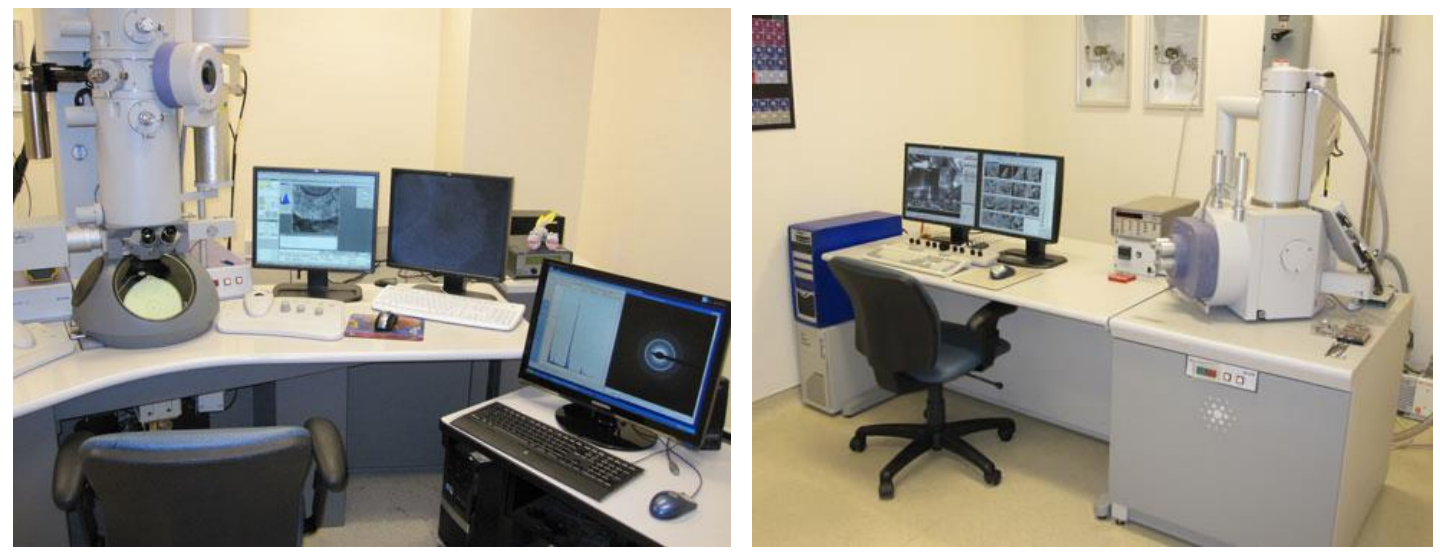

Fig. 2.5 Photos of TEM (left) and SEM (right) which was utilized for the structure and morphology characterization.

\subsubsection{Laser Diffraction Particle Size Analyzer}

A Laser Diffraction Particle Size Analyzer (Microtrac 3500, USA) (Fig. 2.6) was used to determine the size distribution of the agent. This device has a tri-laser, multi-detector, and multiangle optical system. Algorithms that utilize Mie compensation and Modified Mie calculations for non-spherical particles were used for the particle size calculation. Measurement capability from 0.02 to 2800 microns can be achieved with the precision within $0.6 \%$. 


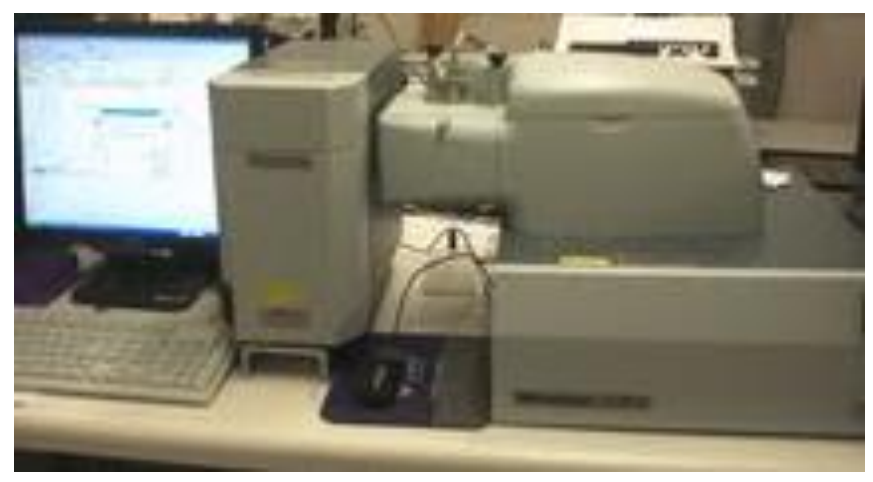

Fig 2.6 Laser Diffraction Particle Size Analyzer - Microtrac 3500.

\subsubsection{Atomic Absorption Spectroscopy}

Atomic absorption spectrometry (AAS) quantifies the absorption of ground state atoms in the gaseous state. The atoms absorb ultraviolet or visible light and make transition to higher electronic energy level. The analyte concentration is determined from the amount of absorption. In order to quantify the GNP concentration at each synthesis step and GNP up-load efficiency by PLGA particles, an AAS method was used. Firstly, $0.5 \mathrm{~mL}$ aqua regia (3 to 1 ratio of $70 \% \mathrm{HCl}$ and $37 \% \mathrm{HNO}_{3}$ ) was added to $1 \mathrm{~mL}$ as synthesized GNP solution and left for 10 minutes. Next, the resulted solution was diluted to have 5\% acid content and its gold component was quantified using an AAS device (PerkinElmer AAnalyst 800, USA) (Fig. 2.7). To quantify the GNP up-load efficiency, PLGA/PFH-GNP particles were mixed with acetone for two hours to dissolve the PLGA shell. Then, the $0.5 \mathrm{~mL} 40 \% \mathrm{HF}$ and $1 \mathrm{~mL}$ aqua regia was added to the solution and heated at about 100 degree Celsius in a teflon container for two hours to dissolve silica coating and degrade the gold nanoparticles into gold atoms. Then $5 \mathrm{~mL} \mathrm{4 \%} \mathrm{Boric} \mathrm{acid} \mathrm{was} \mathrm{added} \mathrm{to} \mathrm{the}$ solution. The resulted solution was tested using the AAS device to determine the concentration of gold nanoparticles. 


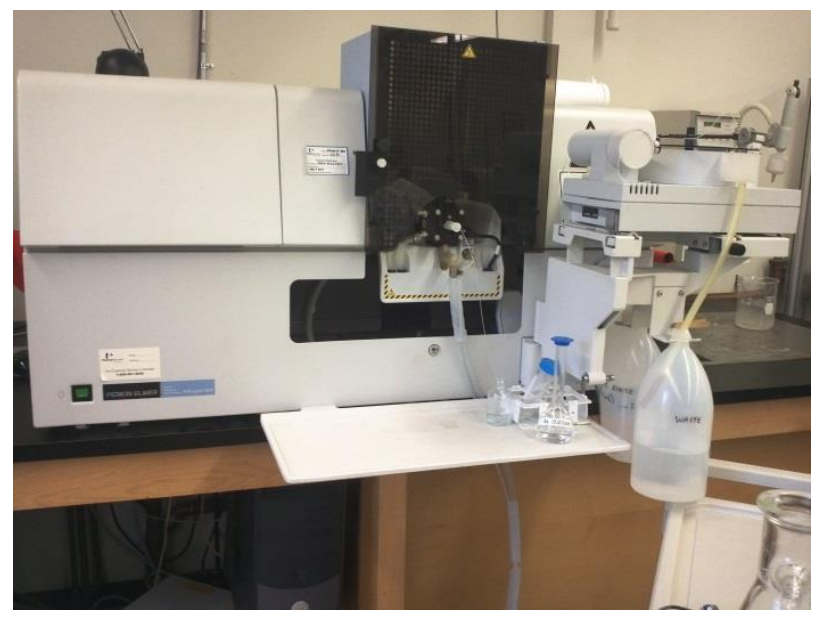

Fig. 2.7 A photograph of the atomic absorption spectrometer that was used to measure the concentration of GNPs in solutions.

\subsubsection{Photoacoustic Microscope}

An acoustic microscope (SASAM, Kibero GmbH, Germany) (Fig. 2.8) was used for all photoacoustic measurements [11]. The microscope consists of three main components: an optical microscope (IX81 Olympus, Japan) with its objectives fixed under the sample stage, a transducer positioned above the sample stage, and a focused laser (Teem Photonics, France) collimated through the side port onto the sample. A $532 \mathrm{~nm}$ laser was used to produce pulses that had a 330 ps pulse width, $4 \mathrm{kHz}$ repetition rate, a $10 \mu \mathrm{m}$ focusing spot size, and a maximum energy of 450 nJ per pulse. The transducer used for this study has a central frequency of $375 \mathrm{MHz}, 42 \%$ bandwidth, and $60^{\circ}$ aperture. Signals were amplified by a $40 \mathrm{~dB}$ amplifier (Miteq, USA) and digitized at $8 \mathrm{GHz}$ (DC252, Agilent, USA). 

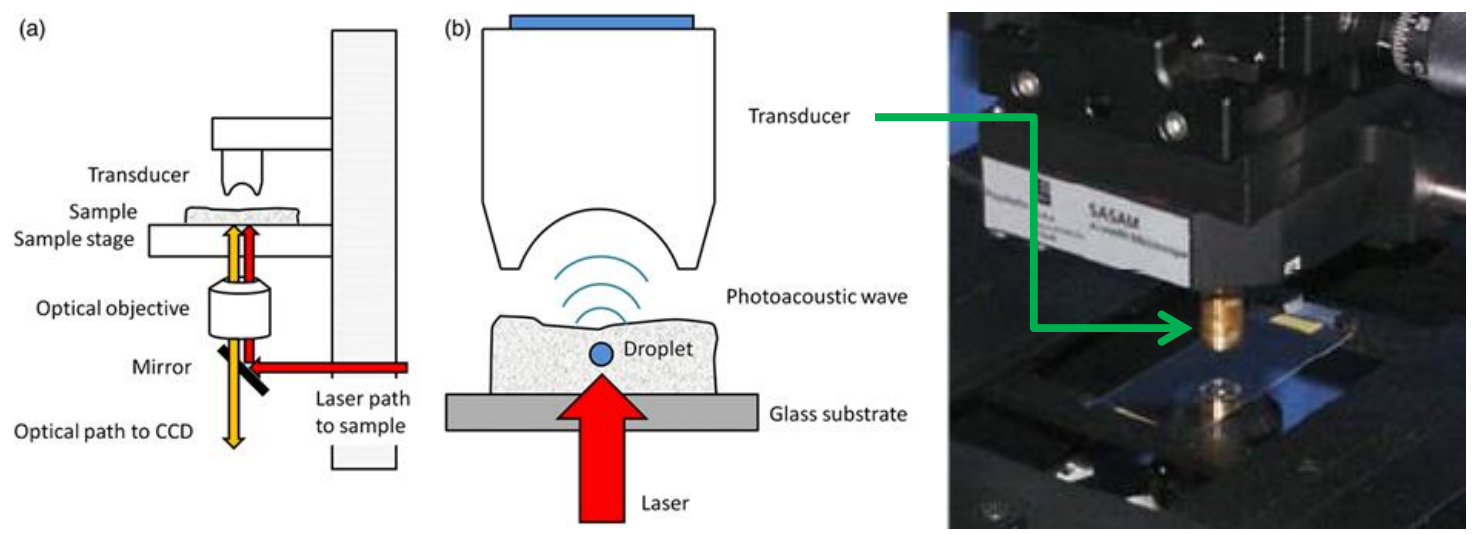

Fig 2.8 Photoacoustic experimental setup, left (a) schematic demonstration of the photoacoustic microscope and middle (b) the geometrical setup; right: a photo of SASAM shows the single-element transducer fixed on top of the sample stage. The optical objectives are under the sample stage. Figure is adapted from [11].

\subsubsection{Photoacoustic Measurements}

The PLGA/PFH-GNP solution were diluted with water and loaded on top of a glass coverslip on the sample stage. Each droplet was centered and aligned with the laser and transducer for photoacoustic measurements. The laser fluence was increased gradually from 40 to $850 \mathrm{~mJ} / \mathrm{cm}^{2}$, while the PA signal amplitudes were measured for each setting. The process was repeated until vaporization occurred or the maximum laser fluence level was reached. The peakto-peak photoacoustic signal amplitudes and vaporization thresholds as a function of laser fluence were recorded. Three types of PLGA/PFH-GNP particles were examined, 2.0 \pm 0.5 , $5.0 \pm 0.5$, and $10 \pm 0.5 \mu \mathrm{m}$ in diameter, each containing $14 \mathrm{~nm}, 35 \mathrm{~nm}$ or $55 \mathrm{~nm}$ (in diameter) gold core nanoparticles enclosed in a $20 \mathrm{~nm}$ silica shell (Fig. 2.9). An average of 10 particles of each type was examined. All measurements were made at $37^{\circ} \mathrm{C}$ to simulate physiological conditions within the human body. 


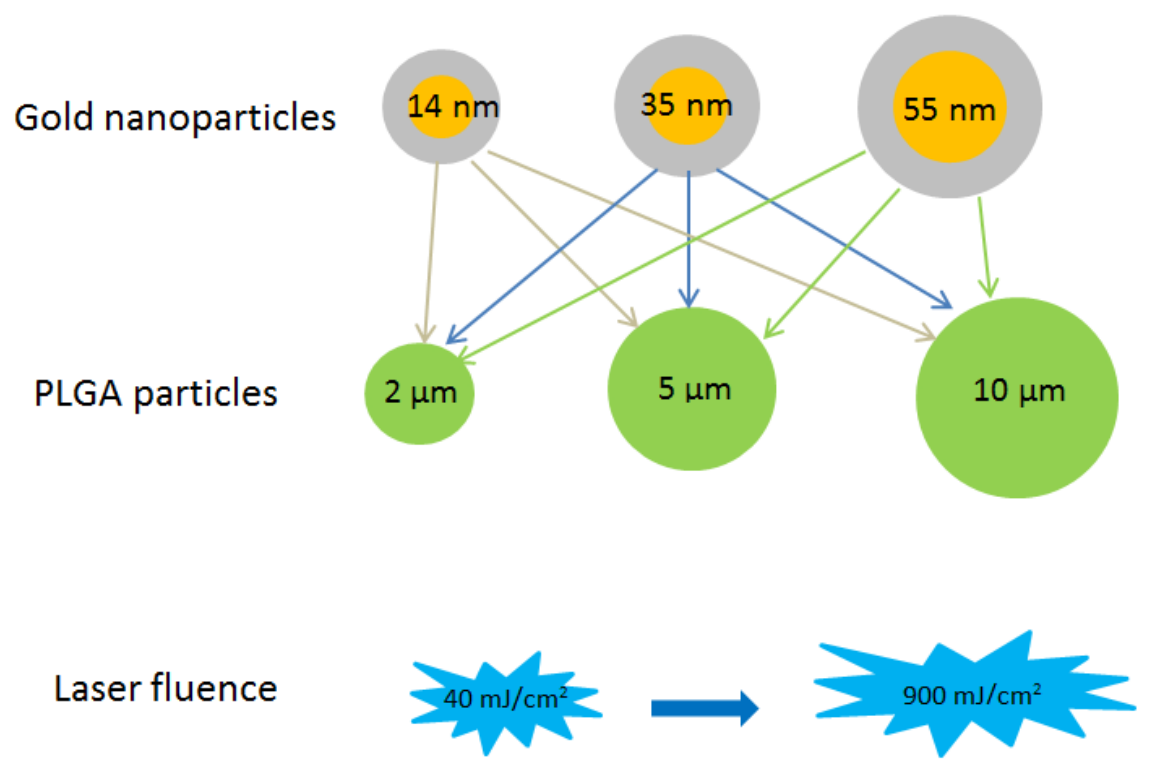

Fig. 2.9 Three sizes of GNPs were used for the synthesis of PLGA/PFH-GNP particles. Three sizes of PLGA particles were chosen for PA signal and vaporization threshold measurements.

\subsection{Results and Discussion}

Results from all parts of the synthesis method and characterization of PLGA/PFH-GNP particles through UV-NIR spectrophotometry, TEM, SEM, a Laser Diffraction Particle Sizer, and PA measurements are presented and discussed in this section.

In this study, gold nanospheres were selected as the optical absorbing material due to their high absorption coefficient around $530 \mathrm{~nm}$, ease of synthesis, and small size permitting high-yield incorporation into micron-scale PLGA particles. A $20 \mathrm{~nm}$ silica coating was chosen to maximize the PA signal strength and the stability of gold nanospheres under laser irradiation [82]. The silica shell also facilitated the GNPs misibilized into the hydrophobic and lipophobic PFH liquid using the ligand exchange technique [133]. Three sizes of GNPs (14 nm, $35 \mathrm{~nm}$, and 55 $\mathrm{nm}$ in diameter) with $20 \mathrm{~nm}$ silica shell each were used for the synthesis of the PLGA/PFH-GNP particles. TEM images in Fig. 2.10 demonstrate good monodispersity of silica-coated GNPs with 
three sizes $(13.9 \pm 2.4,34.9 \pm 2.5$ and $54.5 \pm 4.2 \mathrm{~nm}$ in diameter of gold cores, and $20.0 \pm 5.0 \mathrm{~nm}$ thick silica shells) in aqueous solutions. The overall concentrations are $6.3 \times 10^{12}, 1.6 \times 10^{11}$, and 4.6x $10^{10} \mathrm{GNPs} / \mathrm{ml}$ of solution for 14,35 , and $55 \mathrm{~nm}$ GNPs, respectively (determined from AAS measurement, described in section 2.3.4). TEM results show that there were about 5\% nonspherical GNPs in each batch. This synthesis protocol was very sensitive to reaction conditions and small deviations from the method can create large changes in size. It is of vital importance that all reagents be prepared directly before the reaction.

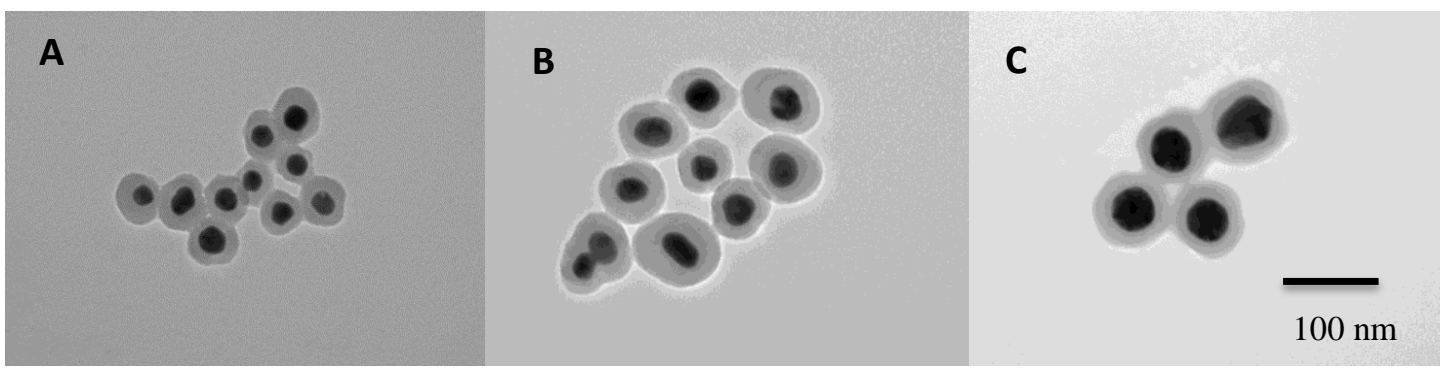

Fig. 2.10 TEM images of silica-coated GNPs, $14 \mathrm{~nm}$ (A), $35 \mathrm{~nm}$ (B), and $55 \mathrm{~nm}$ (C) in diameter (dark areas) with $20 \mathrm{~nm}$ thick silica shells (light gray areas). The scale bar for all figures is $100 \mathrm{~nm}$.

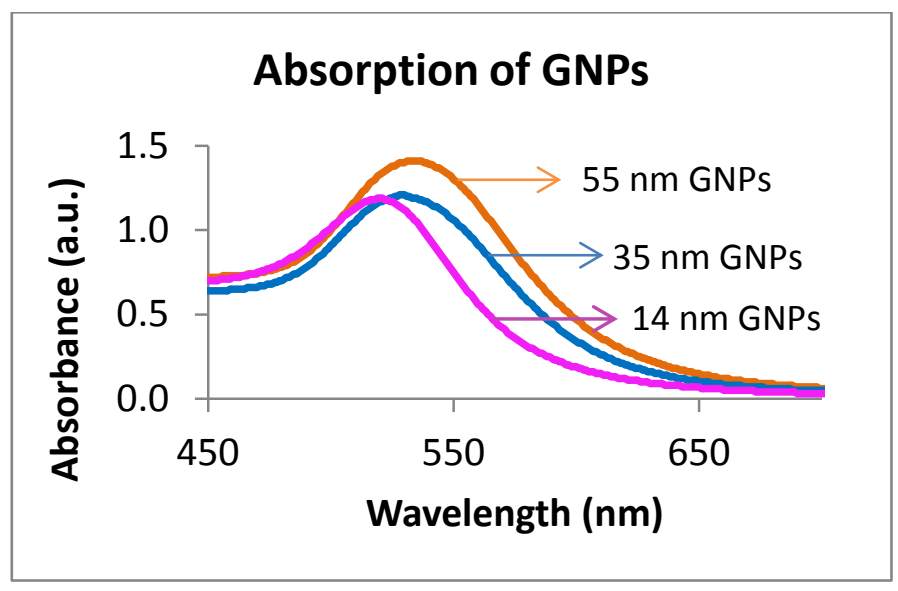

Fig. 2.11 The optical absorption spectra of three sizes of gold nanoparticles $(14,35,55 \mathrm{~nm})$ are shown in magenta, blue and red. 
The optical absorption spectra were measured and recorded (shown in Fig. 2.11). The absorption peak of $14 \mathrm{~nm}$ gold nanoparticle is at $520 \mathrm{~nm}$. As the particle size increased, the absorption peaks demonstrates a red shift. As a result, the peak for $35 \mathrm{~nm}$ and $55 \mathrm{~nm}$ gold nanoparticles is at $526 \mathrm{~nm}$ and $535 \mathrm{~nm}$, respectively. The absorption coefficient amplitude is proportional to the optical absorption cross section of GNPs. The spectrum of $55 \mathrm{~nm}$ GNPs has a largest peak amplitude value comparing to the other two.

The second step in the synthesis method was the phase transfer of GNPs from the aqueous form as synthesized to the organic phase so that they may be suspended in PFH. The described method successfully phase transferred GNPs into the organic phase without altering the optical properties (Fig. 2.12). Therefore, this method is a highly robust procedure to allow GNPs to be encapsulated into PLGA/PFH particles.

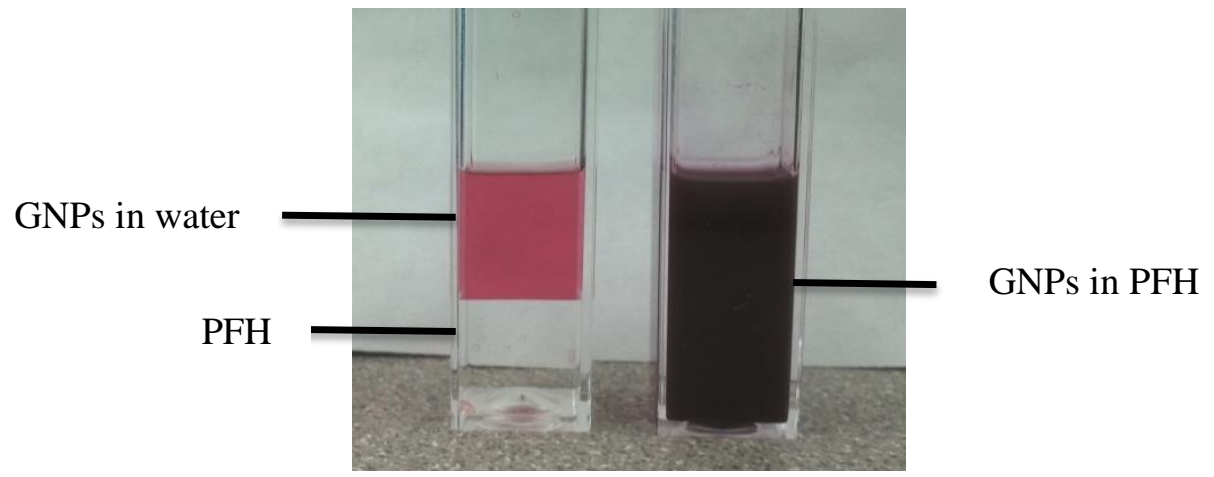

Fig. 2.12 GNPs are in water (left) and organic (right) phase.

After synthesis of PLGA/PFH-GNP particles via a water-oil-water double emulsion technique, several approaches were used to characterize the PLGA/PFH-GNP particles. First, SEM confirmed the spherical morphology of an individual PLGA/PFH-GNP particle (left image in Fig. 2.13) and the existence of a population of varied sizes of PLGA/PFH-GNP particles within a same sample (right image in Fig. 2.13). The particle size range is from 0.2 to $12 \mu \mathrm{m}$ and 
the average size of the PLGA/PFH-GNP particles in this image is around $1 \mu \mathrm{m}$. The shell thickness of PLGA/PFH-GNP particles is about $10 \%$ of the particle diameter.
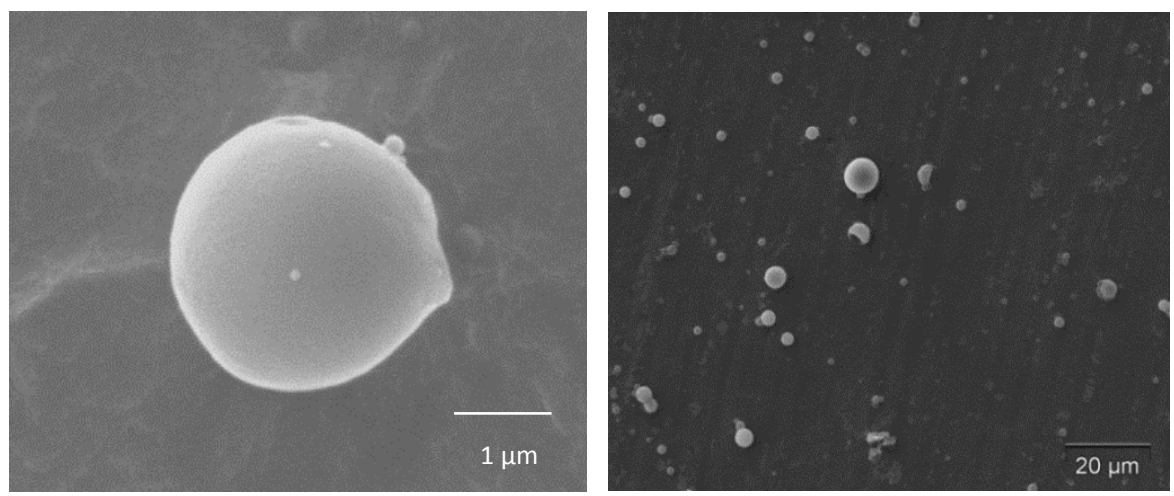

Fig. 2.13 SEM images of PLGA/PFH-GNP particles (PLGA shell thickness is approximately 10\% of the particle diameter). The particle size range is from 0.3 to $12 \mu \mathrm{m}$.

Fig. 2.14 shows TEM images of single PLGA particles loaded with PFH liquid and silicacoated GNPs. The black dots are the GNPs and the gray background is the PLGA shell. Since the fluorinated silica-capped GNPs were well dispersed in the PFH liquid, most of them seem to be located in the core with the PFH liquid, others are mixed in the shell. If the average size of the PLGA particles is $1 \mu \mathrm{m}$, we estimate that on average there are about 468,8 , and 3 GNPs encapsulated in each PLGA particle for 14, 35, and $55 \mathrm{~nm}$ GNPs.
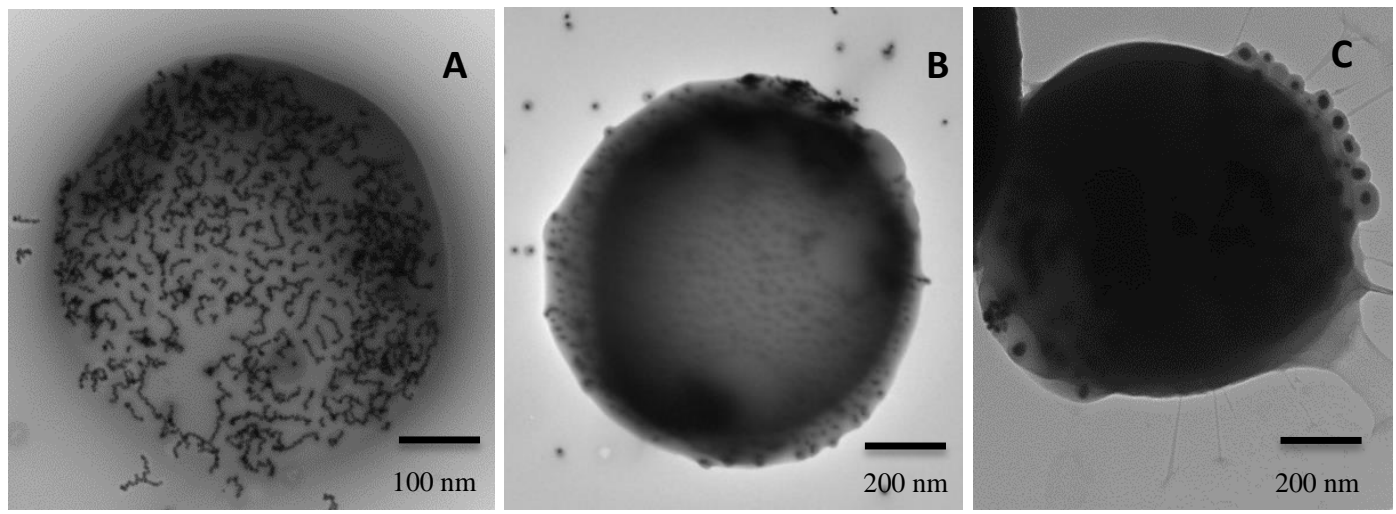

Fig. 2.14 TEM image of a single PLGA particle loaded with PFH liquid and silica-coated GNPs. The black dots are the GNPs. The gray background is the PLGA shell, A-14 nm GNPs, B-35 nm GNPs, and C-55 nm GNPs. 
Second, absorption spectrophotometry was used to measure the optical properties of the PLGA/PFH-GNP particles. The spectrum of a solution of the particles has a peak around $540 \mathrm{~nm}$ with a very broad shape in comparison with a spectrum of the GNP solution alone (Fig. 2.15). The peak shift from $526 \mathrm{~nm}$ to $540 \mathrm{~nm}$ is due to the silica coating [135]. There are several reasons might cause the peak to broaden. First, the concentrated GNPs produce a spherical shell on the PLGA particle which may result in a red shift and broader spectrum. Second, the aggregation of GNPs inside the PLGA particle may also contribute to the broader absorption spectrum. Third, the broad size distribution of PLGA particle (200 nm to $1500 \mathrm{~nm}$ ) may shift the absorption peaks at different wavelength. Last, the coalescence of the PLGA particles might also contribute to the final absorption characteristics.

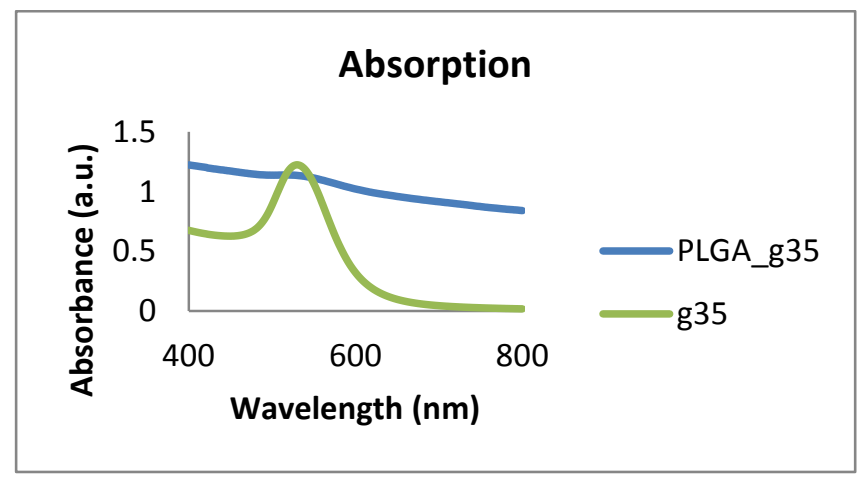

Fig. 2.15 The absorption spectrum of $35 \mathrm{~nm}$ GNP solution and PLGA loaded with $35 \mathrm{~nm}$ GNP solution.

A laser diffraction particle size analyzer was used to confirm the particle diameters. The sample size distribution is given in Fig. 2.16. For the three batches of PLGA/PFH-GNPs, the size distribution of the particles was approximately $0.2 \mu \mathrm{m}$ to $15 \mu \mathrm{m}$ with average size around $0.6 \mu \mathrm{m}$. This initial sizing dependence was correlated to the amount of PLGA and PFH used and the sonication time and power applied during the synthesis process. The lower the concentration of PLGA and PFH, the smaller the particles were on average. 
Optical Image

\section{PLGA/PFH-14 nm GNPs}
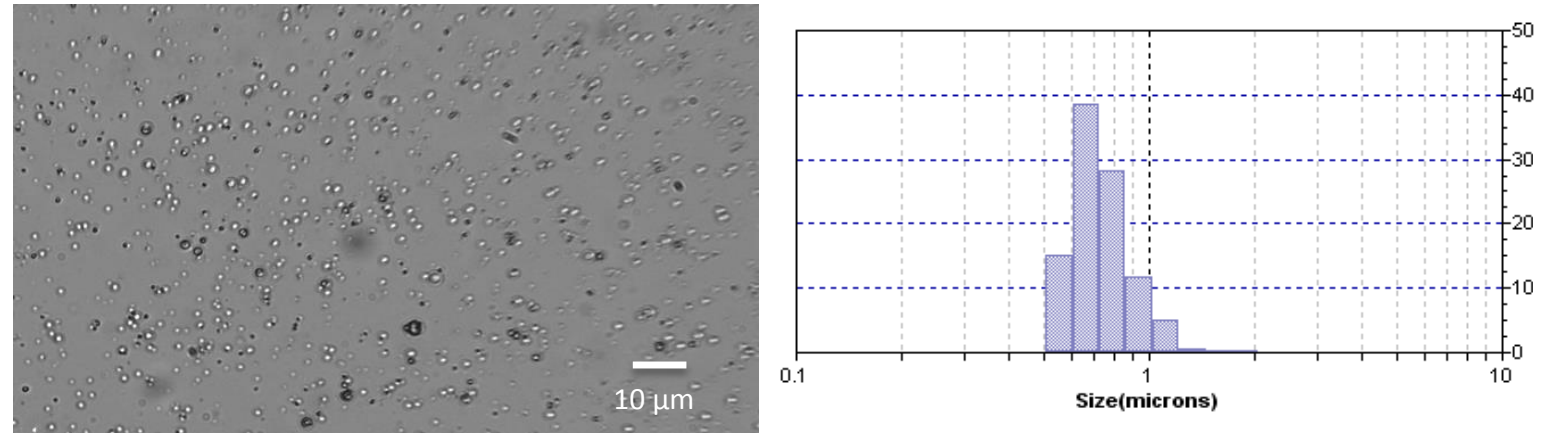

$0.562 \pm 0.091 \mu \mathrm{m}$

\section{PLGA/PFH-35 nm GNPs}
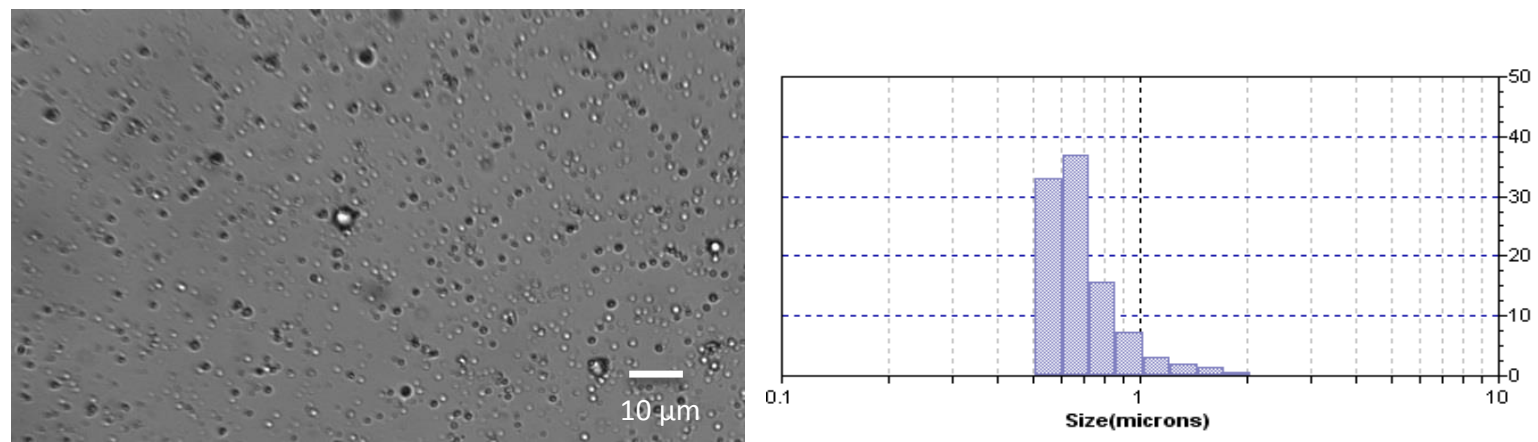

$0.623 \pm 0.131 \mu \mathrm{m}$

\section{PLGA/PFH-55 nm GNPs}
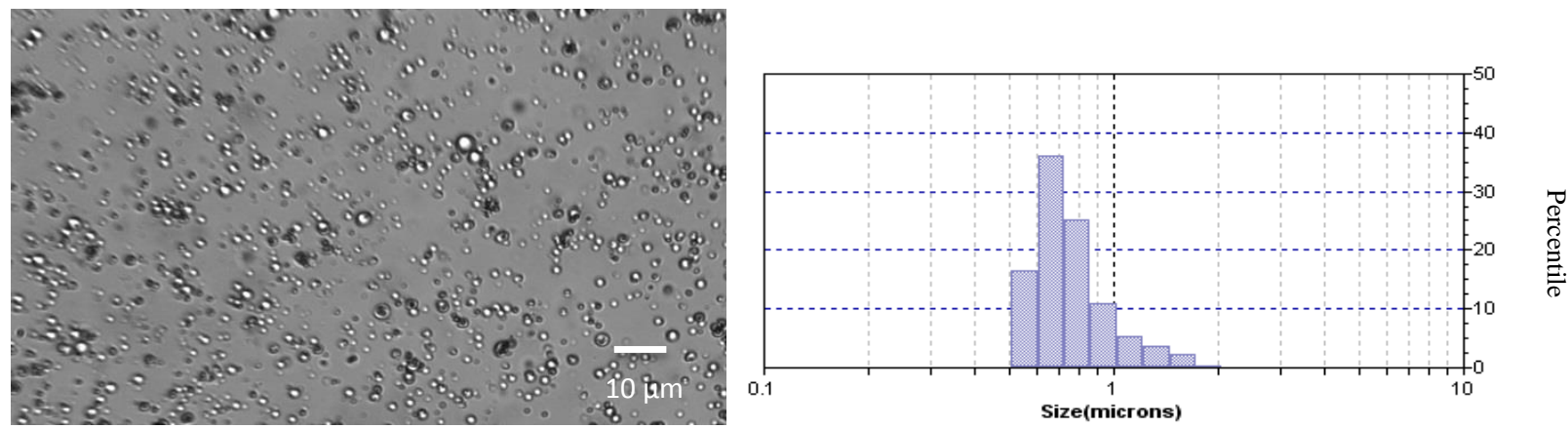

$$
0.680 \pm 0.156 \mu \mathrm{m}
$$

Fig. 2.16 The optical images of three types of PLGA/PFH-GNPs made from three sizes of GNPs (14, 35, $55 \mathrm{~nm})$ and their size distribution. All three images are on the same scale. 

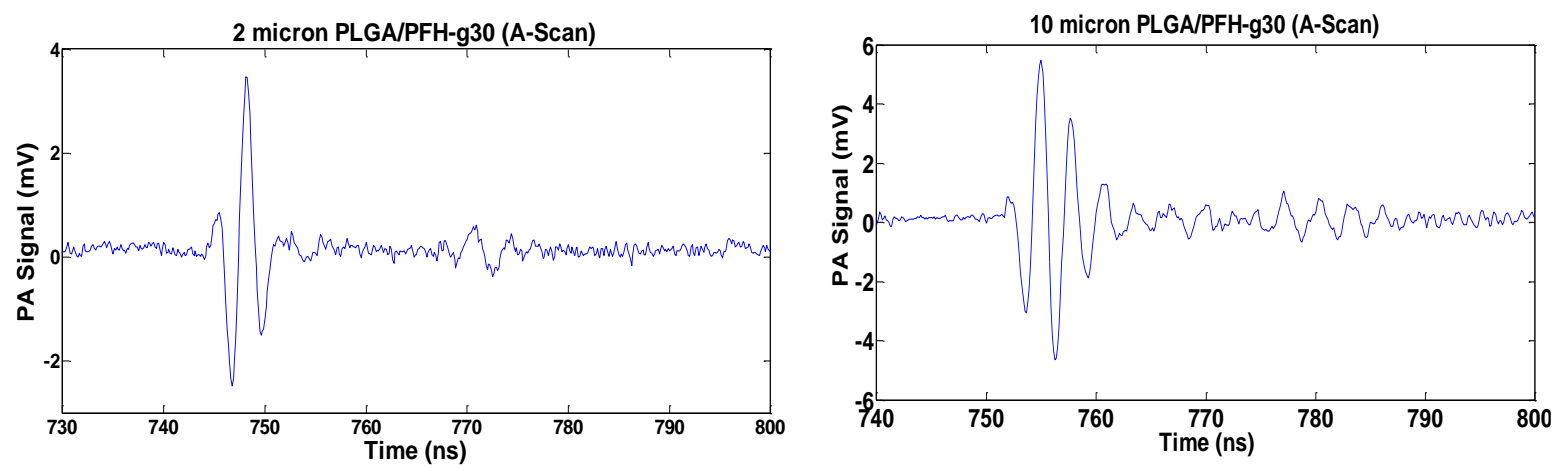

Fig. 2.17 are the photoacoustic signals obtained from 2 and $10 \mu \mathrm{m}$ PLGA/PFH-GNPs.

PA signals and images of PLGA particles were obtained using the SASAM acoustic microscope. The two representative samples of the PA signal of individual PLGA/PFH-GNP particles obtained prior to the vaporization are shown here in Fig. 2.17. The peak-to-peak signal amplitudes were used for the calculation of PA signal intensity as functions of laser energy and particle size. The signal amplitude generated from the $10 \mu \mathrm{m}$ PLGA particle is higher than the one from $2 \mu \mathrm{m}$ particle. The longer signal from the $10 \mu \mathrm{m}$ particle indicates a larger particle size (compared for example with the $2 \mu \mathrm{m}$ particle). Fig. 2.18 shows the optical (left) and PA (middle) images of a single PLGA/PFH-GNP particle loaded with $55 \mathrm{~nm}$ GNPs. Its PA signal (right) was obtained at the center of the particle. The bright pixel intensity in the PA images was due to the strong PA signal generated by the GNPs inside the PLGA particles.
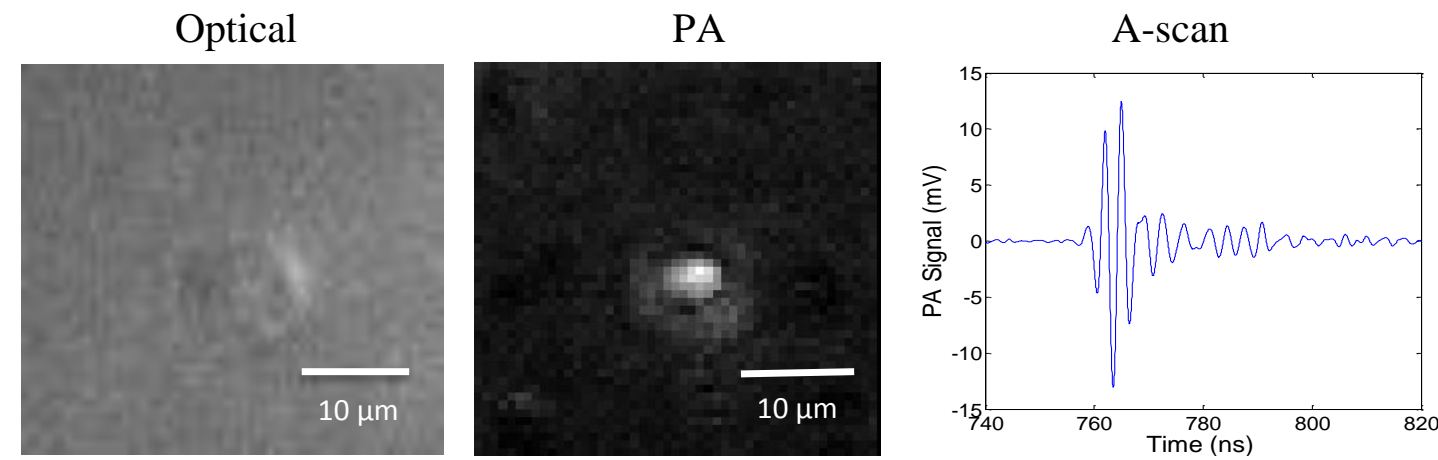

Fig. 2.18 Optical (left) and PA (middle) images of a $10 \mu \mathrm{m}$ PLGA/PFH-GNP particle and its PA signal obtained at the center of the particle. The PA signal intensity (bright pixels) were generated from the GNPs located inside the PLGA particle. 

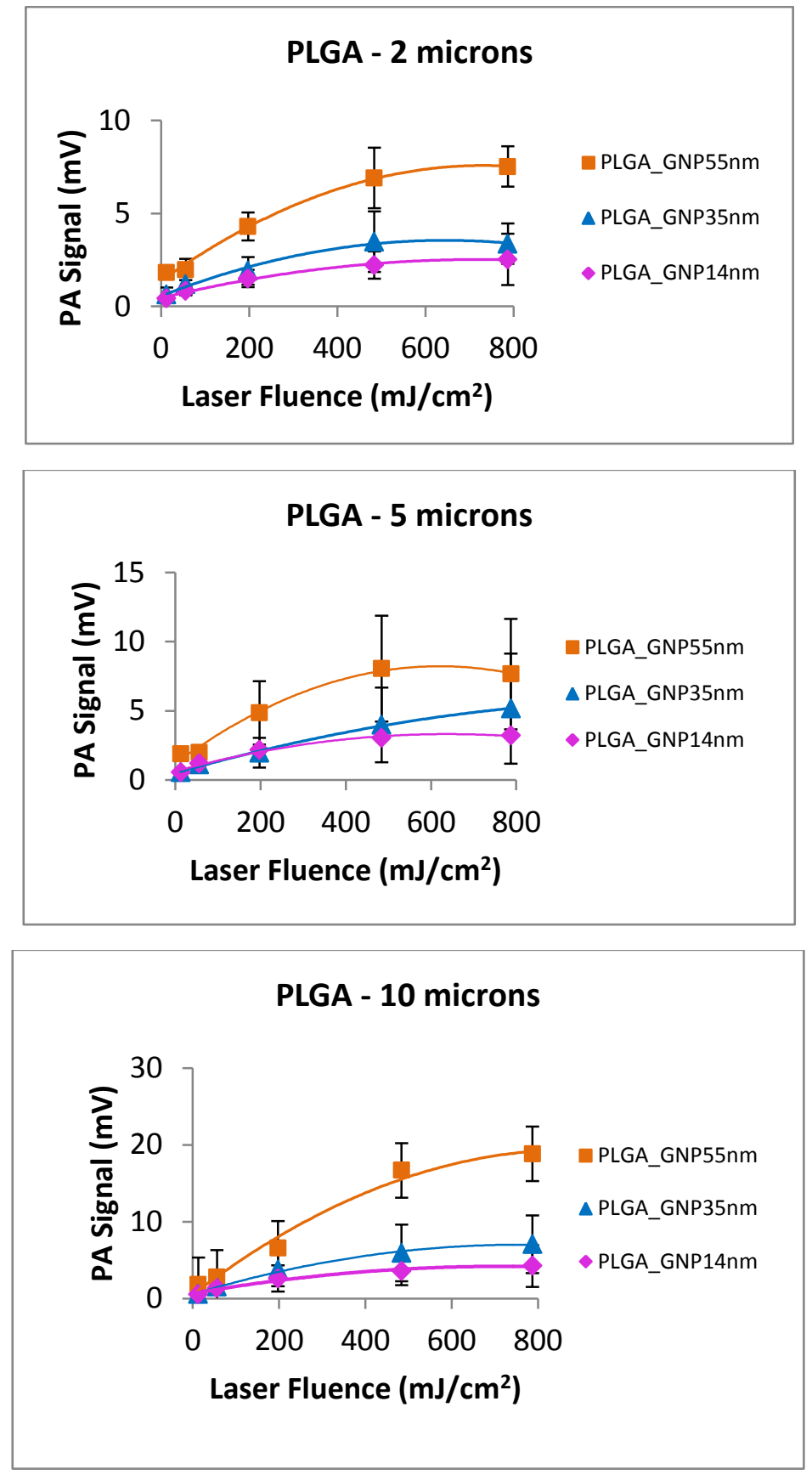

Fig. 2.19 The PA signal as function of the laser fluence for three different particle sizes. Error bars represent the standard deviation of ten measurements on one size of the PLGA particles. 

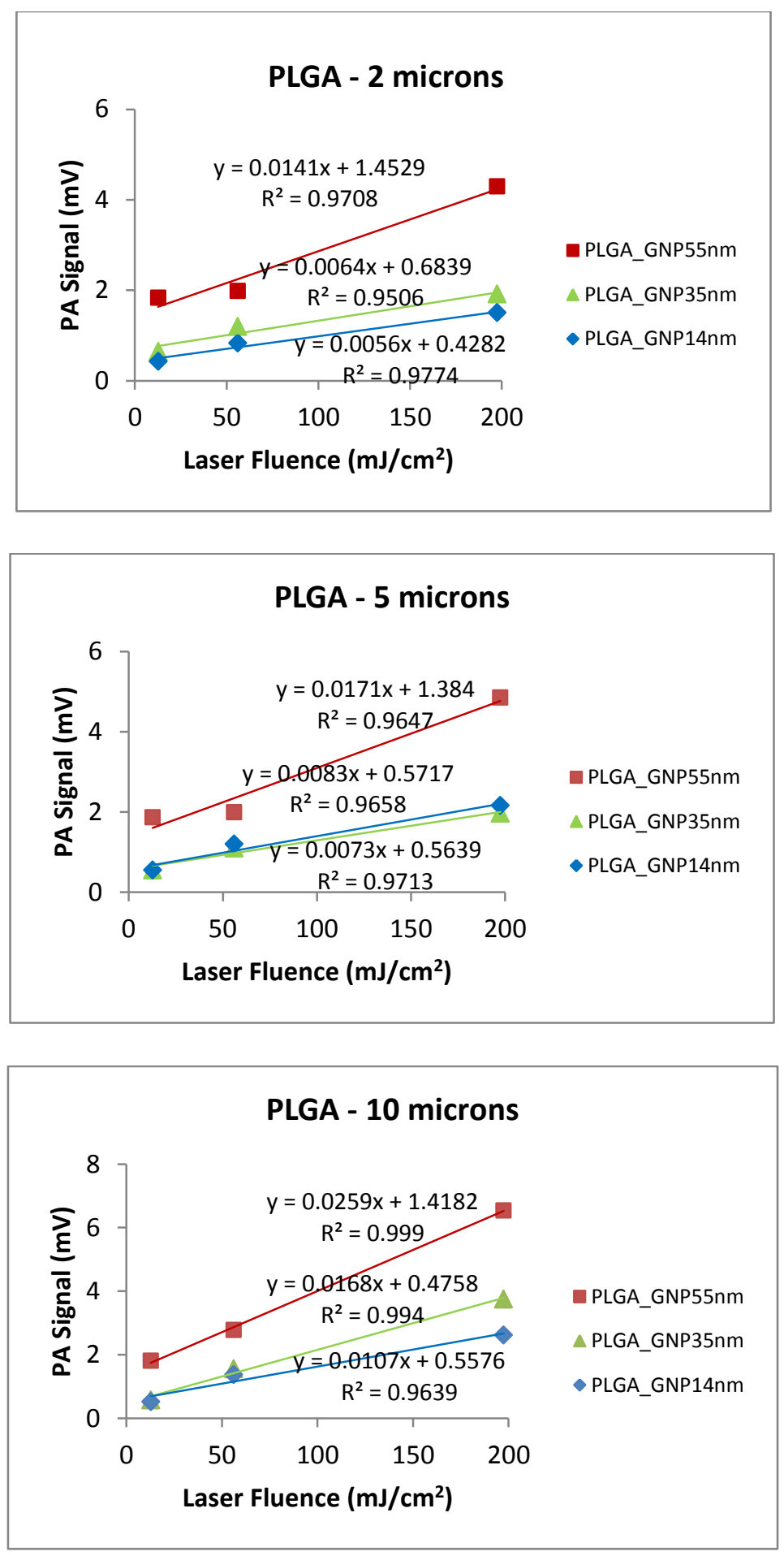

Fig 2.20 At the low energy level $\left(<200 \mathrm{~mJ} / \mathrm{cm}^{2}\right)$, the PA signal is linearly proportional to the laser fluence for three types of PLGA/PHF-GNP particles. 
Fig. 2.19 shows the PA signal as function of laser energy. Each dot in the graph is an average value of 10 independent measurements of individual PLGA particles of a specific size. At low energy level $\left(<200 \mathrm{~mJ} / \mathrm{cm}^{2}\right)$, the signal amplitudes increase linearly with the laser energy. These linear fits are plotted in Fig. 2.20 with an average $\mathrm{R}^{2}$ value larger than 0.95 . The linear relationship between PA signal and laser fluence was predicted by the theory described in Equation (9) (page. The slope of each plot is also correlated with the particle size. According to Equation (9), the slope of the graph is proportional to the GNP absorption coefficient. The larger particle with greater absorption cross section generates the graph with steeper slope than the smaller one does. Our results show for example that the $10 \mu \mathrm{m}$ PLGA particles loaded with 55 $\mathrm{nm}, 35 \mathrm{~nm}$, and $14 \mathrm{~nm}$ GNPs produced lines with slopes of $0.0259,0.0168$, and $0.010 \mathrm{~cm}^{-1}$, respectively.

In Fig. 2.19, at higher energies, the signal intensity stops increasing, potentially because of damage to the GNPs and PLGA particles. GNP damage due to the high laser fluence irradiation has been recorded by Didychuk and his co-workers [136]. The larger the particle size, the higher the PA signal produced. This is because a) more GNPs were encapsulated inside the PLGA particles for the large PLGA particle size and b) a particle of larger size produces a stronger signal. Fig 2.19 also indicates that the larger the GNPs, the stronger the PA signal generated. This is likely because bigger GNPs have a larger absorption across section which would result in a stronger PA signal intensity. 

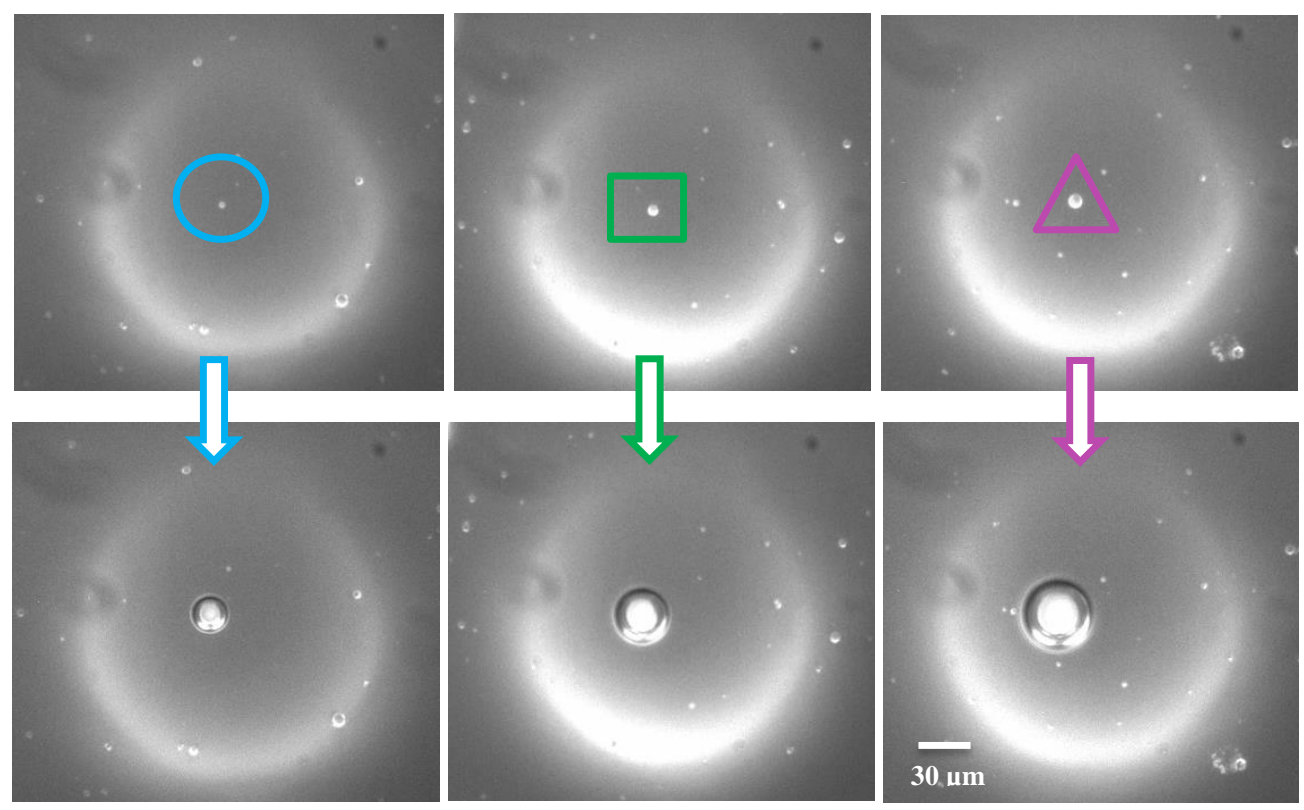

Fig. 2.21 Optical images of PLGA/PFH-GNPs before and after vaporization. The initial particle diameter was 2, 5 and $10 \mu \mathrm{m}$ (top row from left to right). The bubble diameter slowly increased to 20, 30, and 40 $\mu \mathrm{m}$ after 2 seconds (bottom row from left to right). The scale bar is $30 \mu \mathrm{m}$.

Droplet vaporization was observed and recorded as video sequences. Fig. 2.21 shows a vaporization process in which the bubble formed instantaneously with the laser pulse irradiation, then expanded to approximately 10x the original particle size 2 seconds after vaporization. Then the bubble diameter slowly increased over time. It was noticed that the PA signal increased as the laser fluence increased until a peak value was reached before vaporization was triggered. At the moment of the vaporization event, the PA signal reached a maximum value, and then rapidly dropped down to a noise level.

The vaporization results were recorded and plotted in Fig. 2.22. Ten particles were measured for each type of PLGA/PFH-GNP particles. If around 50\% of particles were vaporized, 
then the fluence level was recorded as the vaporization threshold. The arrows indicate the position of the vaporization threshold for each type of PLGA/PFH-GNP particles, which was 850, 690 and $420 \mathrm{~mJ} / \mathrm{cm}^{2}$ for $5 \mu \mathrm{m}$ PLGA/PFH-GNP particles loaded with 14, 35, and $55 \mathrm{~nm}$ GNPs with $20 \mathrm{~nm}$ silica shells, respectively. A lower fluence level was required for PLGA particles with larger GNPs and larger PLGA particles due to the greater absorption cross section of GNPs and greater amount of GNPs encapsulated in the PLGA particles. The fluence rates are rather high due to the lower concentration of GNPs used in this experiment (only one third of original GNP concentration, $2.1 \times 10^{12}, 5.5 \times 10^{10}$, and $1.5 \times 10^{10} \mathrm{GNPs} / \mathrm{ml}$ of 14,35 , and $55 \mathrm{~nm}$ GNPs, respectively). This will be optimized in the future work by adjusting the GNP concentration and shell properties. 

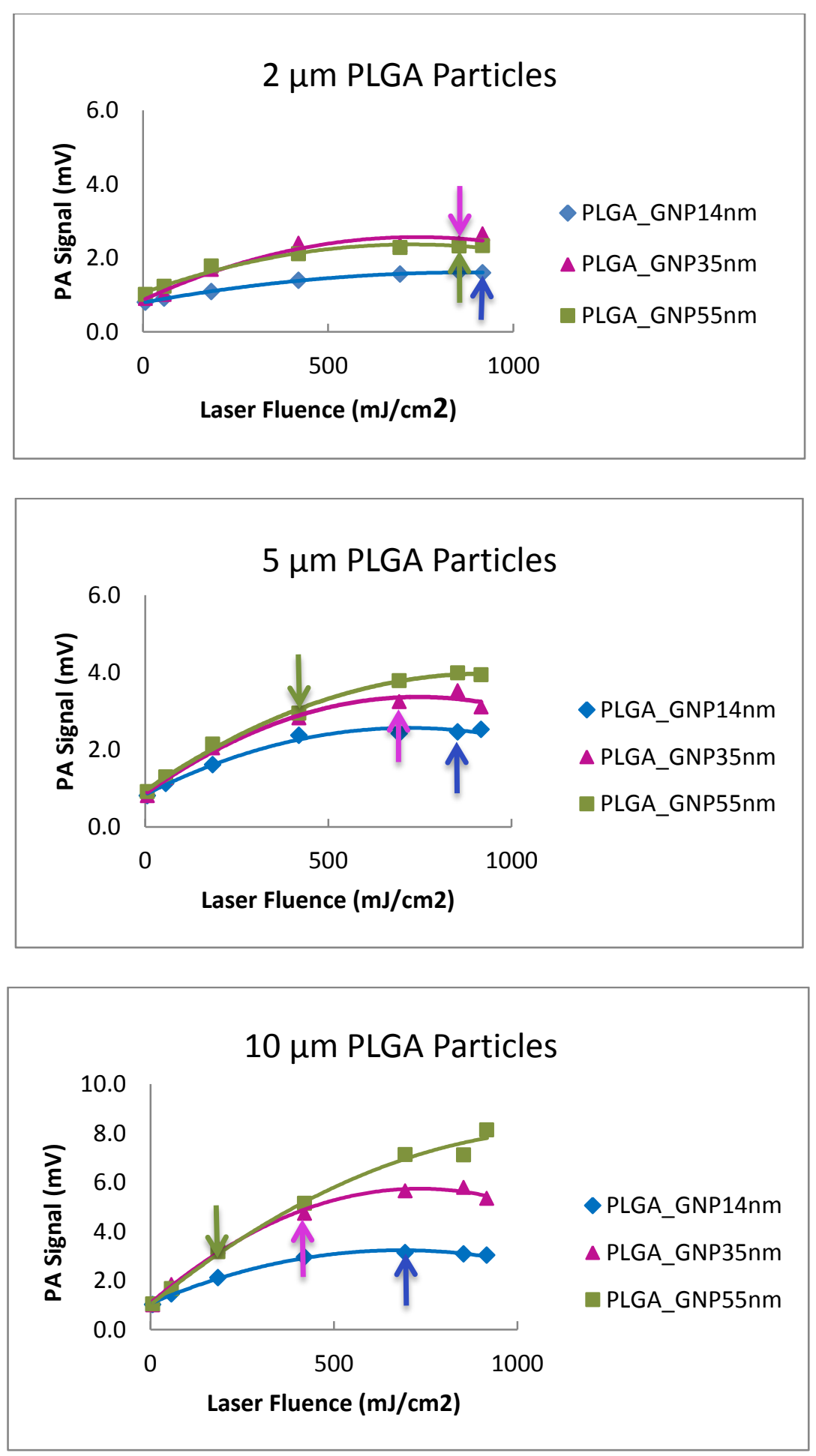

Fig. 2.22 Photoacoustic signal amplitudes and vaporization threshold as functions of laser fluence and GNP sizes. The arrow positions indicate when more than $50 \%$ of the particles vaporized (arrows color coded to particle size). 
Table 1 Quantities of GNPs Concentration in Each Synthesis Process

\begin{tabular}{|c|c|c|c|c|}
\hline Particles & $\begin{array}{c}\text { Diameter } \\
(\mathrm{nm})\end{array}$ & AAS (mg/L) & $\begin{array}{c}\text { Concentration } \\
10 \text { ( }) \\
\text { (GNPs/mL) }\end{array}$ & Ratio \\
\hline g14 & 14 & $11.23 \pm 0.13$ & 6300 & 1 \\
\hline g35 & 35 & $12.21 \pm 0.57$ & 160 & 0.03 \\
\hline g55 & 55 & $13.78 \pm 0.42$ & 46 & 0.01 \\
\hline g14@silica & 14 & $7.61 \pm 0.32$ & 4270 & 0.68 \\
\hline g35@silica & 35 & $10.51 \pm 0.12$ & 137 & 0.86 \\
\hline g55@silica & 55 & $10.67 \pm 0.33$ & 36 & 0.77 \\
\hline & 14 & $2.03 \pm 0.15$ & 1140 & 0.18 \\
\hline PLGA_g14 & 35 & $1.40 \pm 0.13$ & 18 & 0.12 \\
\hline PLGA_g35 & 35 & $1.65 \pm 0.07$ & 6 & 0.12 \\
\hline PLGA_g55 & 55 & & & \\
\hline
\end{tabular}

The concentrations of GNPs in each synthesis step were calculated according the AAS results which are shown in Table 1. Because the initial amount of gold salt used for three sizes GNP synthesis were the same, the smaller the GNPs produced, the larger the concentration of GNPs in the final solution. The GNP concentration was $6.30 \times 10^{12}, 1.60 \times 10^{11}, 4.60 \times 10^{10} \mathrm{NPs} / \mathrm{mL}$ for the14, 35, and $55 \mathrm{~nm}$ GNPs respectively. The yields of GNPs at each step were calculated by comparing the final GNP concentration with the initial GNP concentration. For the silica-coating process, the GNP yield was $67.7 \%, 86.1 \%$, and $77.4 \%$ for the $14,35,55 \mathrm{~nm}$ GNPs, respectively. The GNP upload efficiency by the PLGA was $18.1 \%, 11.5 \%$, and $12.0 \%$ for the $14,35,55 \mathrm{~nm}$ GNPs, respectively. 
In conclusion, PLGA/PFH-GNP particles were designed and synthesized using a reproducible synthesis method for PA imaging. Their PA response was measured while varying the GNP size, the PLGA particle size and laser energy. The flexibility of the PLGA/PFH-GNP platform design allows for easy adaptation to a specific imaging application. In the next chapter, the therapeutic ability of PLGA/PFH-GNPs will be explored in vitro study. 


\section{Chapter 3 - In Vitro Study}

The objective of the research described in this chapter is to determine the effects that PLGA/PFH-GNP particles will have upon cell uptake. Firstly, the cytotoxicity of the particles as they are internalized by the cell will be examined. Secondly, the cytotoxicity after localized vaporization of the PLGA/PFH-GNP particles inside the cells will be examined. In order to accomplish these goals, several studies were undertaken. Firstly, cells were incubated with PLGA/PFH-GNP particles for 24 hours to determine if the PLGA/PFH-GNP particles caused cytotoxic effects. Next, the passive internalization by cancer cells was investigated. Finally, the effects of the localized vaporization of the PLGA/PFH-GNP particles inside the cells were studied in terms of effect on cell viability and membrane disruption.

The research described in Chapter 2 has shown PLGA/PFH-GNP particles to be suitable contrast agents for photoacoustic imaging. Furthermore, these particles allow photoacoustic imaging via vaporization also, a photoacoustic phenomenon that produces large amplitude signal as compared to thermal expansion: this occurs only at the time of the rapid expansion during the initial phase change. However, the vaporization of PLGA/PFH-GNP particles needs to be studied in vitro to ensure that PLGA/PFH-GNP particles do not induce biological side effects before the vaporization event. While each component of the PLGA/PFH-GNP particles are thought to be biologically inert or minimally cytotoxic [137]-[139], the cytotoxicity of these components when combined into PLGA/PFH-GNP particles needs to be studied.

PLGA/PFH-GNPs are intended to be used for combined imaging and therapy. While they are introduced to the body to target the diseased tissue (either through the EPR effect or active 
targeting), they will also accumulate within other parts of the body, such as the liver and spleen. These organs are part of the body's phagocyte system that endocytoses foreign bacteria and colloidal particles [140]. PLGA/PFH-GNP particles must not affect healthy cells during the treatment. One method to quantify any potential cytotoxicity effects through passive interactions is via cell culture studies using PLGA/PFH-GNPs incubated with cells and reagents that change properties based on cellular enzymatic activity.

\subsection{Methods}

Experiments using cells culture were designed to determine the interactions of PLGA/PFH-GNPs with cells. The two cell culture lines were used in these experiments, MCF7 and MDA-MB-231 (breast adenocarcinoma). MDA-MB-231 cell line was chosen for its high level of expression of the EGFR (endothelial growth factor receptor) [141]. EGFR is overexpressed in triple-negative or basal breast cancer, which accounts for approximately up to $15 \%$ of all breast cancers but remains one of the most difficult to treat [142][143]. Human epidermal growth factor receptor 2 (HER2) is over-expressed in MCF7 cell line [143]. For the next step of the experiments in future work, the EGFR and HER2 are going to be selected as targets for the PLGA/PFH-GNPs. The cells were purchased from Sigma and maintained in a humidified cell incubator at $37{ }^{\circ} \mathrm{C}$ and 5\% $\mathrm{CO}_{2}$ with Dulbecco's Modified Eagle Media comprising $4500 \mathrm{mg}$ glucose/L, L-glutamine, $\mathrm{NaHCO}_{3}$, and sodium pyruvate with $10 \%$ fetal bovine serum, $1 \%$ HEPES buffer, and $1 \%$ penicillin-streptomycin. Three experiments were performed including a cell cytotoxicity assay to determine the particle cytotoxicity without vaporization, a study of the passive internalization of PLGA/PFH-GNPs by the cells, and a study of the vaporization effects inside the cells caused by pulsed laser irradiation. 


\subsubsection{Passive Cell Internalization of PLGA/PFH-GNP Particles}

Human breast carcinoma MDA-MB-231 cells and MCF7 cells were used for the experiments. Initially, a million cells were seeded in a $75 \mathrm{~mL}$ cell culture flask and left in the incubator for 24 hours. PLGA/PFH-GNP particles $(200 \mu \mathrm{L}, 50 \mu \mathrm{g} / \mathrm{mL})$ were added to the flask. After 6-hour incubation, the cells were washed with PBS $(\mathrm{pH}=7.4)$ to completely dislodge loosely attached and free particles in the medium. Cell pellets were collected in a $1 \mathrm{~mL}$ polystyrene tube by centrifugation and fixed with $10 \%$ formalin and $75 \%$ alcohol. The cell pellets were sliced to $100 \mathrm{~nm}$ thick specimens for TEM imaging. For PA imaging experiments, $1 \times 10^{5}$ cancer cells were placed in a $22 \mathrm{~mm}$ diameter glass bottom cell culture dish and incubated with DiI labeled PLGA/PFH-GNP particles $(50 \mu \mathrm{L}, 50 \mu \mathrm{g} / \mathrm{mL})$ for 6 hours, Then the cells were washed with PBS $(\mathrm{pH}=7.4)$ to completely remove loosely attached and free particles in the medium. The cells were then used for PA study.

For the confocal laser scanning experiments, $1 \times 10^{5}$ cancer cells were planted on a microscope slide in a $60 \mathrm{~mm}$ diameter cell culture dish and incubated with the fluorescent dye FITC ( $40 \mu \mathrm{L}, 8 \mu \mathrm{g} / \mathrm{mL})$ in the media for 36 hours. Then DiI labeled PLGA/PFH-GNP particles $(50 \mu \mathrm{L}, 50 \mu \mathrm{g} / \mathrm{mL}$ ) were added to the dish and incubated for 6 hours. Fluorescent dye Hoechst $(20 \mu \mathrm{L}, 10 \mu \mathrm{g} / \mathrm{mL}$ ) was added to the dish for $15 \mathrm{~min}$. The cells were washed with PBS several times to completely remove loosely attached and free particles in the medium. The cells then were fixed with $10 \%$ formalin and covered with a glass coverslip. After fixation, the cells with DiI labeled particles were observed under a confocal laser scanning microscopy (LSM700, ZEISS, Germany) (Fig. 3.1). The confocal optical sections of cells at different depth were taken to investigate the PLGA/PFH-GNP internalization by cancer cells. 


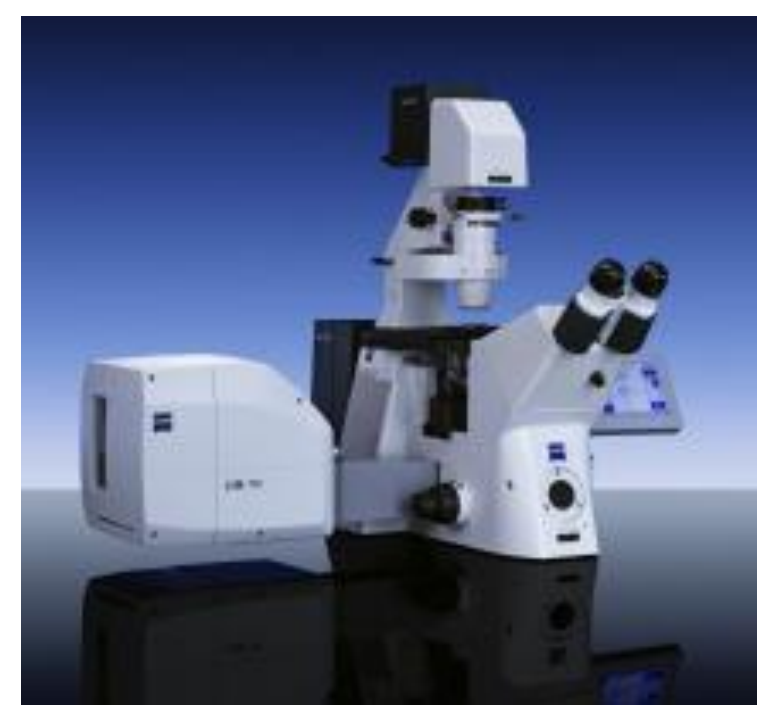

Fig. 3.1 A front view of a confocal laser scanning microscope.

\subsubsection{PLGA/PFH-GNP Particle Cytotoxicity}

The cell cytotoxicity of PLGA/PFH particles was tested using an 3-(4,5-dimethylthiazol2-yl)-2,5-diphenyltetrazolium bromide (MTT) assay. A 96-well plate was seeded with approximately $8000 \mathrm{MCF} 7$ or MDA-MB-231 cells per well, and incubated at $37^{\circ} \mathrm{C}$ and $5 \% \mathrm{CO}_{2}$ for 24 hours. The media was aspirated, and the new media containing three concentrations of PLGA/PFH-GNPs $(22,67,200 \mu \mathrm{g} / \mathrm{mL})$ were add to the non-control wells. Each condition was repeated in six wells to allow for statistical analysis. The plate was incubated for another 24 hours at $37{ }^{\circ} \mathrm{C}$ and $5 \% \mathrm{CO}_{2} .50 \mu \mathrm{L}$ of bleach was added to the positive control wells. The solution from each well was aspirated, and MTT solution (100 $\mu \mathrm{L}, 0.84 \mathrm{mg} / \mathrm{mL})$ was added into each well. The plate was returned to the incubator for 45 minutes. Next, the MTT solution was aspirated, and $100 \mu \mathrm{L}$ of DMSO was added to each well. The absorbance was read at $590 \mathrm{~nm}$ on the 96-well microplate absorbance reader (iMark, BIO-RAD, USA). This experiment was repeated for three times. The absorbance reading of each MTT measurement in wells containing 
solutions with treated cells was subtracted from the reading without MTT treatment to determine the relative viability values. A multivariate analysis of variance was used to determine statistically significant different mean viabilities between the control wells and the wells incubated with varying concentrations of PLGA/PFH-GNPs. Plots were created showing viability relative to the control cells that were only incubated with normal cell culture media.

\subsubsection{Photoacoustic Measurements}

Five thousand MDA cells were seeded in a $22 \mathrm{~mm}$ diameter glass-bottom cell culture dish and incubated for 24 hours. PLGA/PFH-GNP particles labeled with DiI dye were added into the dish and left in the incubator for 6 hours. Next, 3,3'-dioctadecyloxacarbocyanine perchlorate (DiO) dye $(100 \mu \mathrm{L}, 50 \mu \mathrm{g} / \mathrm{mL})$ was added to the cell culture dish and left in the incubator for 30 minutes. Then the cells were washed with PBS several times, Fluorescent dye Hoechst $(20 \mu \mathrm{L}$, $10 \mu \mathrm{g} / \mathrm{mL}$ ) was added to the cell culture dish. Then the cell culture dish was placed in the acoustic microscope. Optical and fluorescence images of MCF7 cells containing DiI labeled PLGA/PFH-GNP particles were obtained. Next, the cells were irradiated with the $532 \mathrm{~nm}$ laser pulse with energy of $380 \mathrm{~nJ}$ while a c-scan image was acquired. All measurements were made at $37^{\circ} \mathrm{C}$ to simulate physiological conditions within the human body. Then the photoacoustic images of cells were compared with fluorescence images to ensure that the photoacoustic signal originated from locations coincident with the locations revealed with fluorescence imaging. 


\subsubsection{Mechanical Damage from Vaporization of PLGA Particles on Cells}

Five thousand MCF7 cells were seeded in a $22 \mathrm{~mm}$ diameter glass bottom cell culture dish. PLGA/PFH-GNP particles were added into the dish and incubated for 6 hours. After the cells were washed with PBS several times, $300 \mu \mathrm{L}$ of $500 \mathrm{nM}$ Propidium iodide (PI) solution was added to the cell culture dish. Then the cell culture dish was placed in the acoustic microscope. MCF7 cells containing PLGA/PFH-GNP particles were irradiated with the laser to examine the vaporization effects of the PLGA/PFH-GNP particles on single cells. All measurements were made at $37^{\circ} \mathrm{C}$ to simulate physiological conditions within the human body. Optical and fluorescence images were obtained before and after the vaporization to examine any changes in cell morphology and structure and damage due to the vaporization events.

\subsection{Results and Discussion}

\subsubsection{Passive Internalization of PLGA/PFH-GNP Particles}

After 6 hours incubation of MCF7 or MDA cells with the PLGA/PFH-GNP particles, the particles were internalized by the cells. The TEM of an ultrathin section in Fig. 3.2A shows a PLGA/PFH-GNP adherent to the surface of the cell or in the process of internalization into vesicles; and Fig. 3.2B displays the PLGA/PFH-GNPs particle internalization inside a cytoplasmic vesicle. The black dots are the GNPs (white arrow). The white area is the hollow core of the PLGA formed during the fixation process (orange arrow). The dark area pointed by the blue arrow is due to the artifact during the fixation process. 

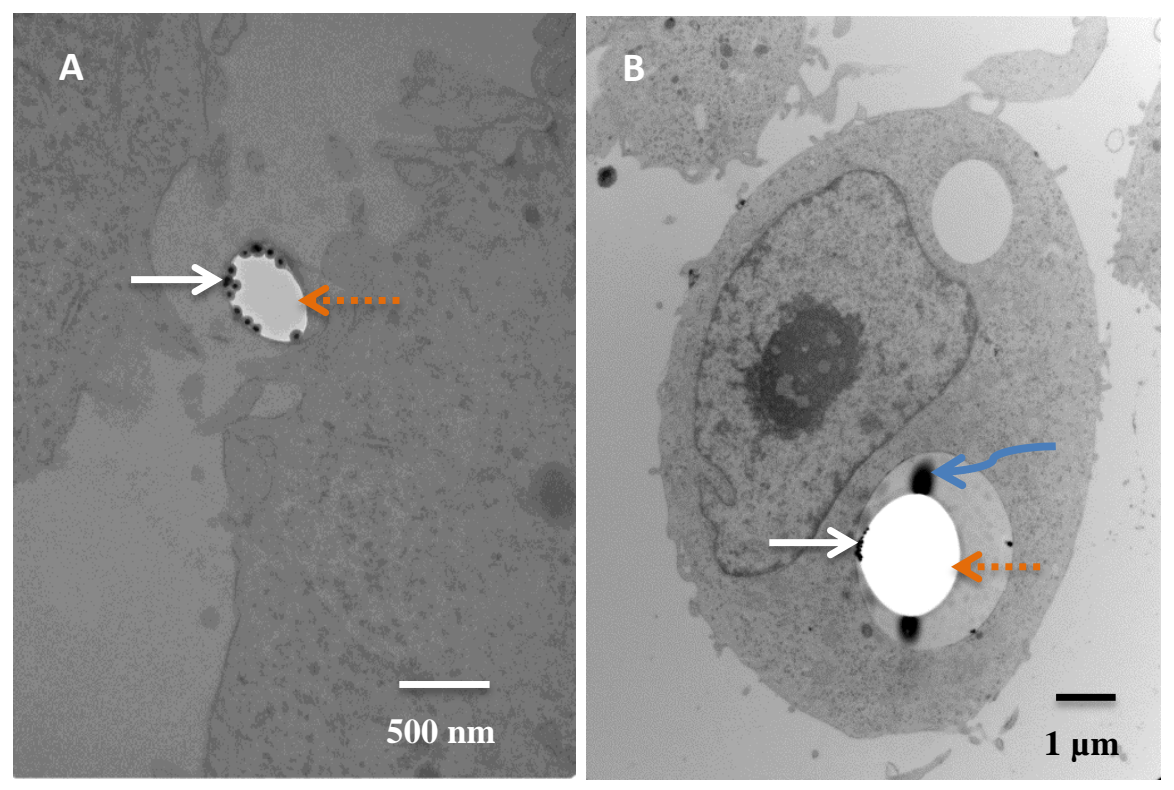

Fig. 3.2 TEM images of a single PLGA/PFH-GNP particle adherent to the surface of the cell and internalized in a cytoplasmic vesicle. The black dots are the GNPs (solid white arrows). The white area is the hollow part of the PLGA particle (dash orange arrows). The dark area (curved blue arrow) is the artifact.

In Fig. 3.3 images of MDA cells (top panel) and MCF7 cells (bottom panel) loaded with DiI-labeled PLGA/PFH-GNP particles as a result of passive internalization are shown. The images are the optical section obtained at cell centers using a confocal laser scanning microscope (section 3.1.1). The cytoplasm is shown in green due to the FITC stain (Fig. 3.3A); nuclei emit blue due to the Hoechst fluorescence (Fig. 3.3B); and DiI labeled PLGA/PFH-GNP particles are shown in red fluorescence (Fig. 3.3C). Fig. 3.3D are the composite images of A, B and C. As the images indicate, a significant amount of DiI-labeled PLGA/PFH-GNP particles were phagocytized by the cancer cells (Fig. 3.2D), and stayed in the cytoplasm. By examining the fluorescence images at difference locations on a microscope slide, we estimated that there were about $25 \%$ of the cell population exhibited this phenomenon. 

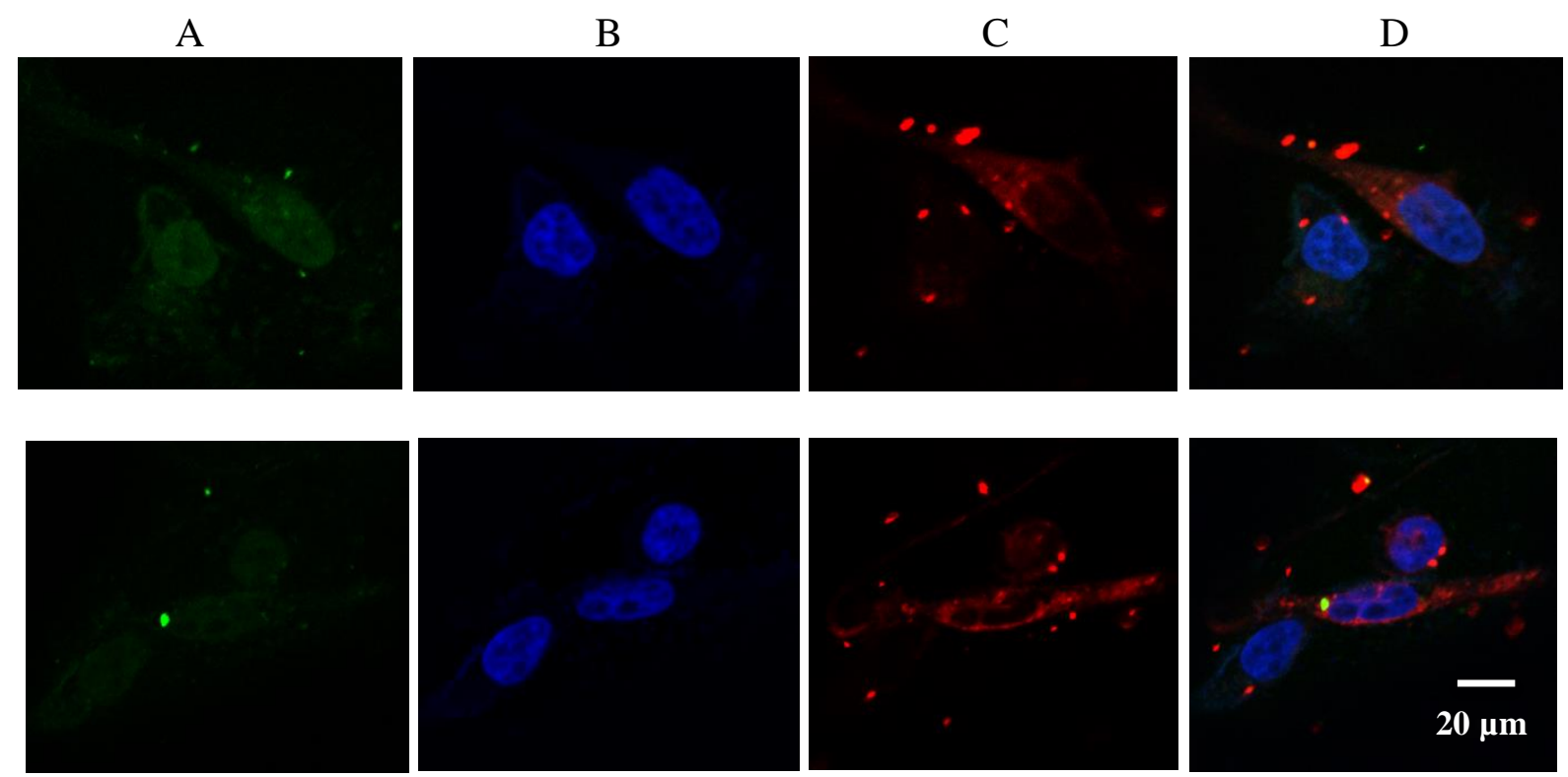

Fig. 3.3 Confocal fluorescence images of PLGA/PFH-GNP particles uptake by MCF7 cells (top row) and MDA cells (bottom row). The PLGA/PFH-GNP particles are labeled by DiI dye in red and are localized in the cell cytoplasm. The nuclei are stained blue by Hoechst. The cytoplasm is stained green by FITC.

\subsubsection{PLGA/PFH-GNP Particle Cytotoxicity}

The cellular cytotoxicity of PLGA/PFH-GNPs when passively incubated with cells for 24 hours was investigated. The relative viability of the cells as compared to control cells is presented. The MTT assay measures cell viability by determining the metabolic reduction of yellow MTT tetrazolium salt to a water insoluble purple formazan. Stronger metabolic activity will produce a darker purple. After incubation with three concentrations of PLGA/PFH-GNP particles $(22,67$, and $200 \mu \mathrm{g} / \mathrm{mL})$ for 24 hours, the MTT assay showed that the cell viability is not affected by the presence of the PLGA/PFH-GNP particles (Fig. 3.4). 


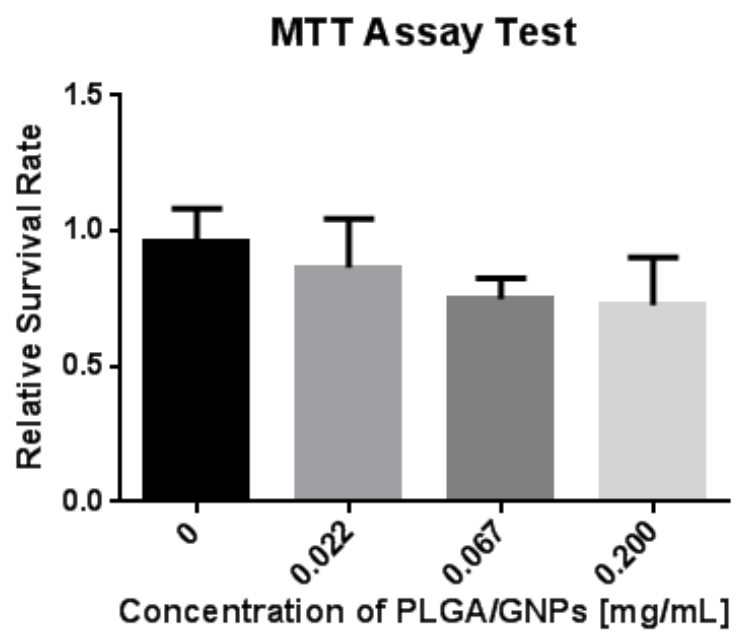

Fig. 3.4 Cytotoxicity test result using the MTT assay. The viability of cells incubated with three concentrations of PLGA/PFH-GNP particles was similar to the control group. Error bars represent the standard deviation of 18 duplicates of each group.

\subsubsection{Photoacoustic Measurements}

MDA cells labeled with DiO and Hoechst were incubated with DiI labeled PLGA/PFHGNP particles for 6 hours to investigate the PLGA/PFH-GNP particle distribution inside the cells and the potential of these particles as PA contrast agents. DiO is suitable for cell membrane and cytoplasm stain, and Hoechst is widely used cell nucleus stain. Fig. 3.5 shows the representative images of MDA cells loaded with PLGA/PFH-GNP-DiI particles. In the superposed fluorescence images, the cell nuclei stained with Hoechst show in blue, and the cytoplasm labeled with DiO show in green. The PLGA/PFH-GNPs labeled by DiI show in red are located in the cytoplasm. In the superposed PA images, cells generate strong PA signal (shown in red) at the location of the PLGA/PFH-GNPs. There is no PA signal at other parts of the cytoplasm, which is confirmed by the fluorescence images. The PA signal strength was generated by the GNPs located inside the PLGA particles. The outlines of the cells in the superposed PA image 
were obtained from the scattered acoustic waves by the cell surface and reflected acoustic waves inside the transduce cavity.

Optical

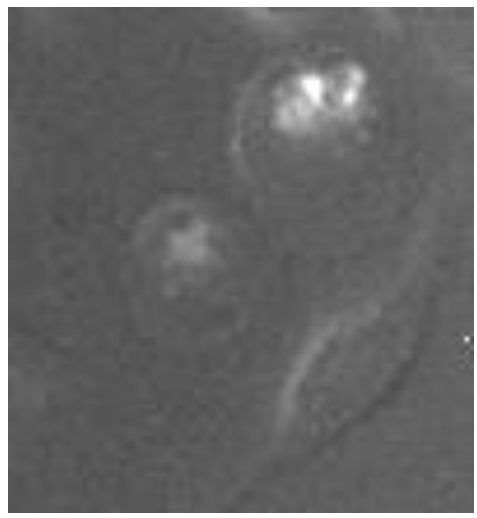

Fluorescence
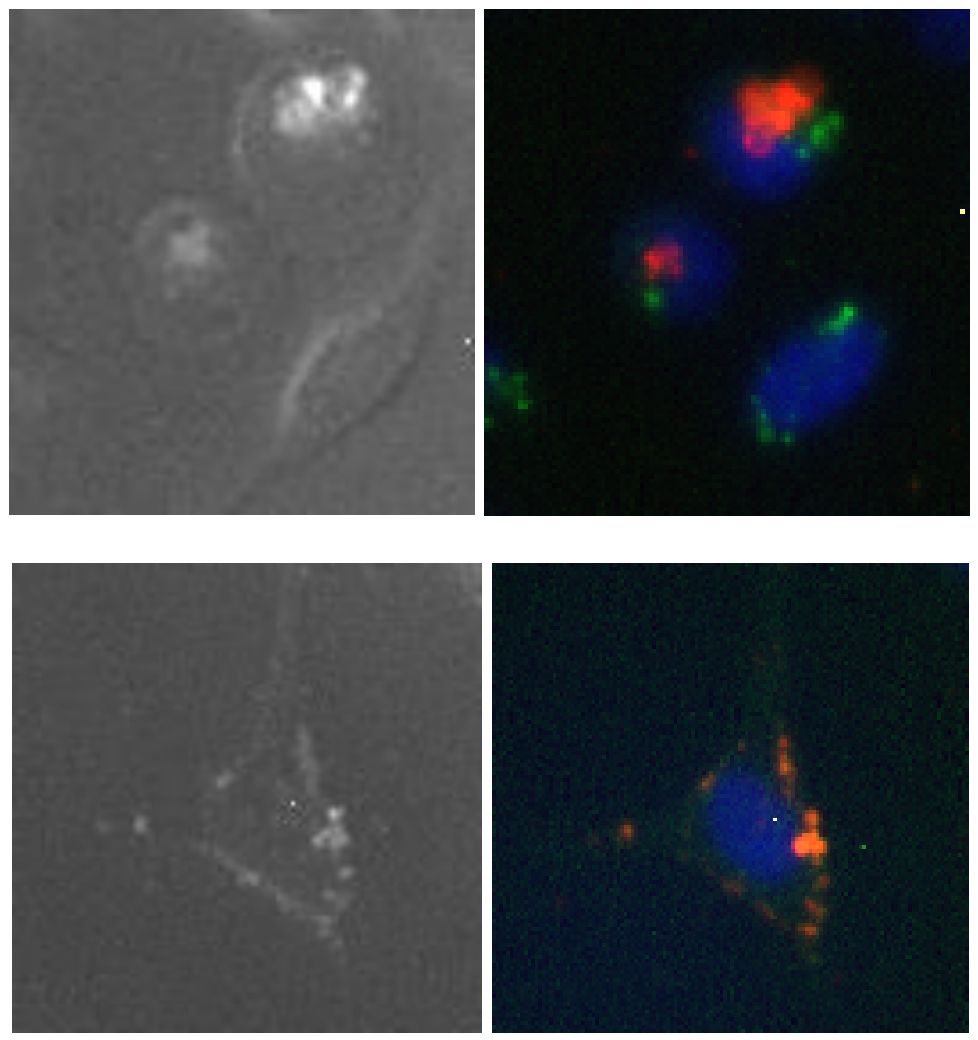

PA
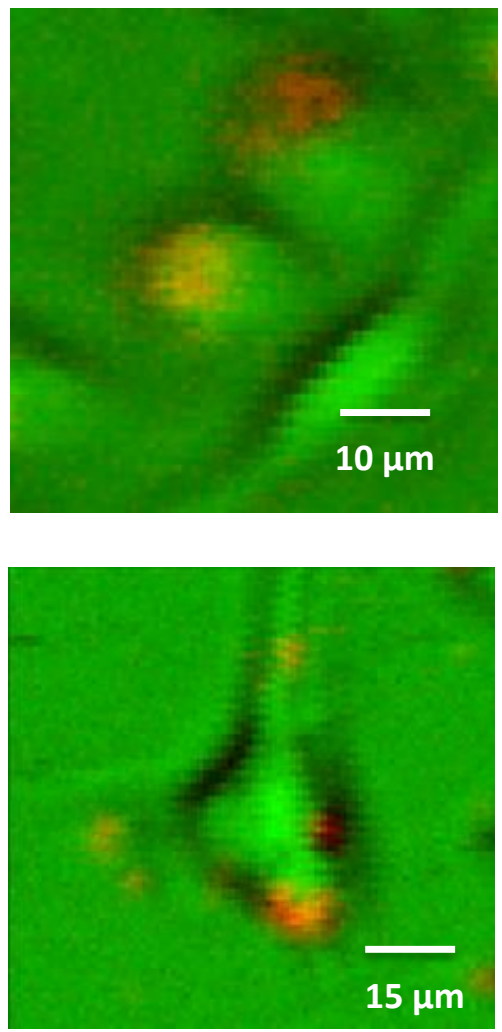

Fig 3.5 Images of MDA cells (top row) and MCF7 cells (bottom row) loaded with DiI labeled PLGA/PFH-GNPs. The PLGA/PFH-GNP particles are shown in bright white in the optical image, and in red in the fluorescence and PA images. In the fluorescence image, cell nuclei are shown in blue due to the Hoechst stain and cell plasma is shown in green by $\mathrm{DiO}$ stain.

Fluorescence and PA images generated by the PLGA-PFH-GNP-DiI particles inside the cells are shown in Fig. 3.6 (top row). The pixel intensity profiles at the cross lines for both images are plotted using Matlab software (shown in the bottom row in Fig. 3.6). The plots indicate that the intensity profiles obtained at the same location inside the cell from two images are quite coincident. The fluorescence intensity profiles obtained at the PLGA/PFH-GNPs location show sharper and narrower shapes comparing with the profiles generated from PA 
image. The fluorescence profiles also show greater signal-to-noise ratios than PA profiles do. This is because the fluorescence signals detected by the fluorescence microscope were based on the DiI dye located in the PLGA. On the other hand, the signal obtained by the acoustic microscope was based on the absorption of GNPs located inside the PLGA particles. The size of the fluorescence intensity of DiI does not depend on the size of the PLGA particle and GNPs, but the PA signal does.

Fluorescence
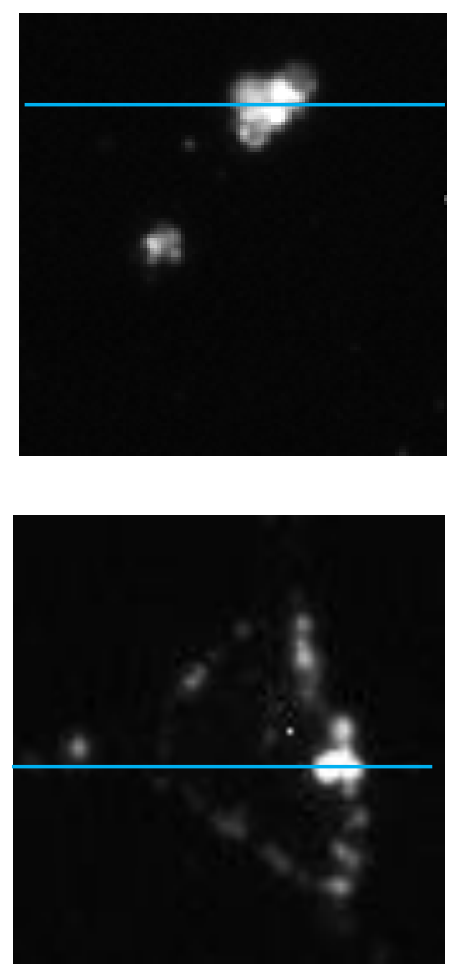

PA
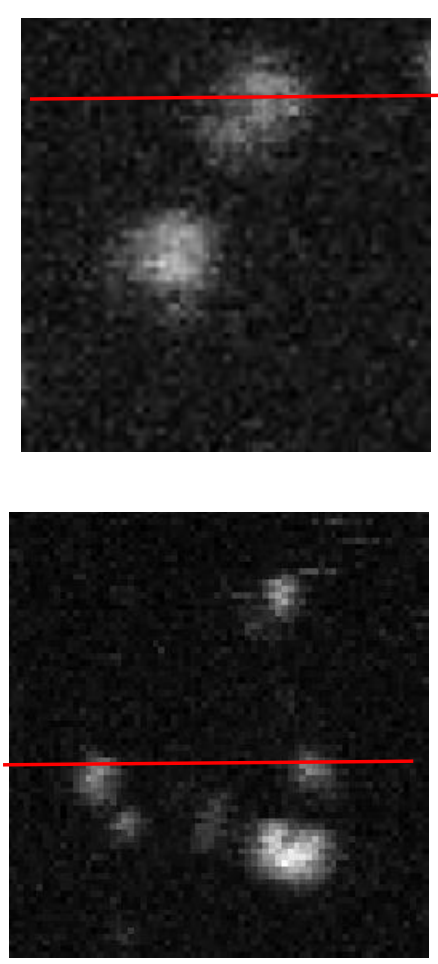

Line Profile
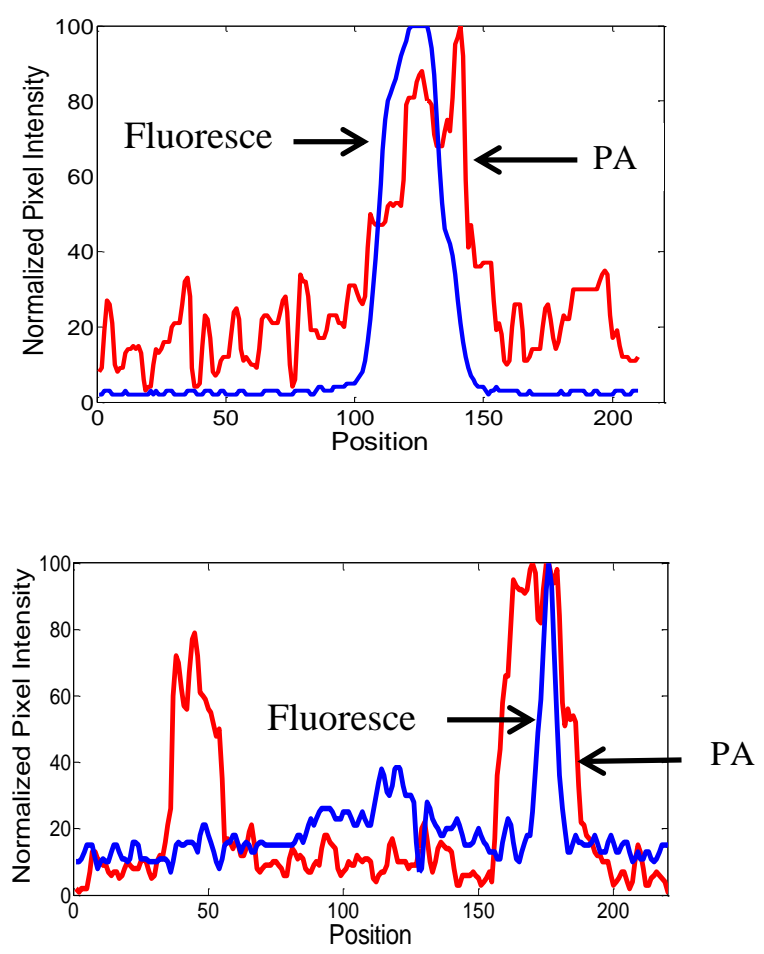

Fig. 3.6 Pixel intensity profiles plotted at the cross lines of fluorescence (blue) and PA (red) images of MDA cells (top row) and MCF7 cell (bottom row).

Fig 3.7 shows two A-scan PA signals recorded at two spots from the PA image. The RF signal obtained at the yellow cross where the PLGA/PFH-GNPs located shows a significant signal-to-noise ratio comparing to the RF signal generated at the blue cross where there is no 
PLGA/PFH-GNPs appeared in the image. This is a representative result from multiple measurements which confirm that the PLGA/PFH-GNP particles can be used as PA contrast agents.

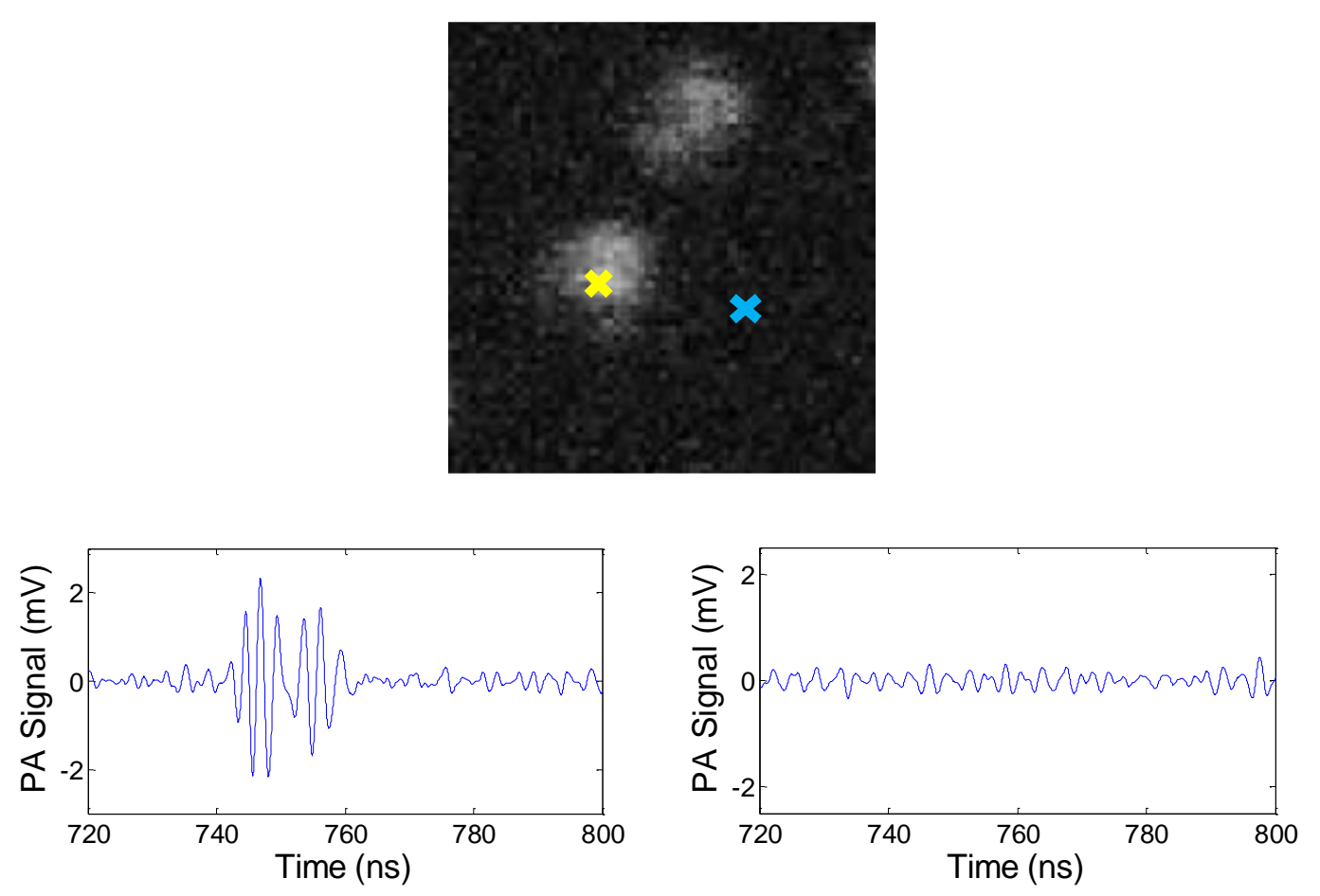

Fig 3.7 Bottom left: an A-scan PA signal obtained at the PLGA/PFH-GNPs location (yellow cross) in the top PA image; bottom right: an A-scan PA signal obtained at the blue cross from the PA image.

\subsubsection{Mechanical Damage from Vaporization of PLGA/PFH-GNP Particles on MCF7 Cells}

Propidium iodide (PI) is membrane impermeable, and excluded from viable cells. If the cell membrane is damaged, PI will bind to the nucleic acids and fluoresce. Upon laser irradiation at fluence level around $680 \mathrm{~mJ} / \mathrm{cm}^{2}$, above $90 \%$ microsized PLGA/PFH-GNP particles within the cell were vaporized, resulting in bubble formation which was observed under the SASAM microscope. In some cases, the bubble remained trapped within the cell, slowly expanding over time (Fig. 3.8A). The bubble exerted pressure within the cell, and the cell slowly retracted (white arrows, Fig. 3.8A). Membrane integrity was lost upon bubble formation, PI fluorescence was 
observed within 20 seconds (Fig. 3.8B). Some of the cells were eventually lifted off the substrate by the bubble.

These results suggest that vaporization inside the cancer cells can cause cell membrane integrity loss, resulting mechanical damage to the cell which further causes cell death (necrosis). The vaporization alone can serve as therapeutic purpose without drug assistance. This can be enhanced by adding therapeutic drug payloads into the particles and attaching cell-specific ligands on the surface of the particles. Future work will be focused on active targeting and controlled drug delivery in vitro and in vivo.
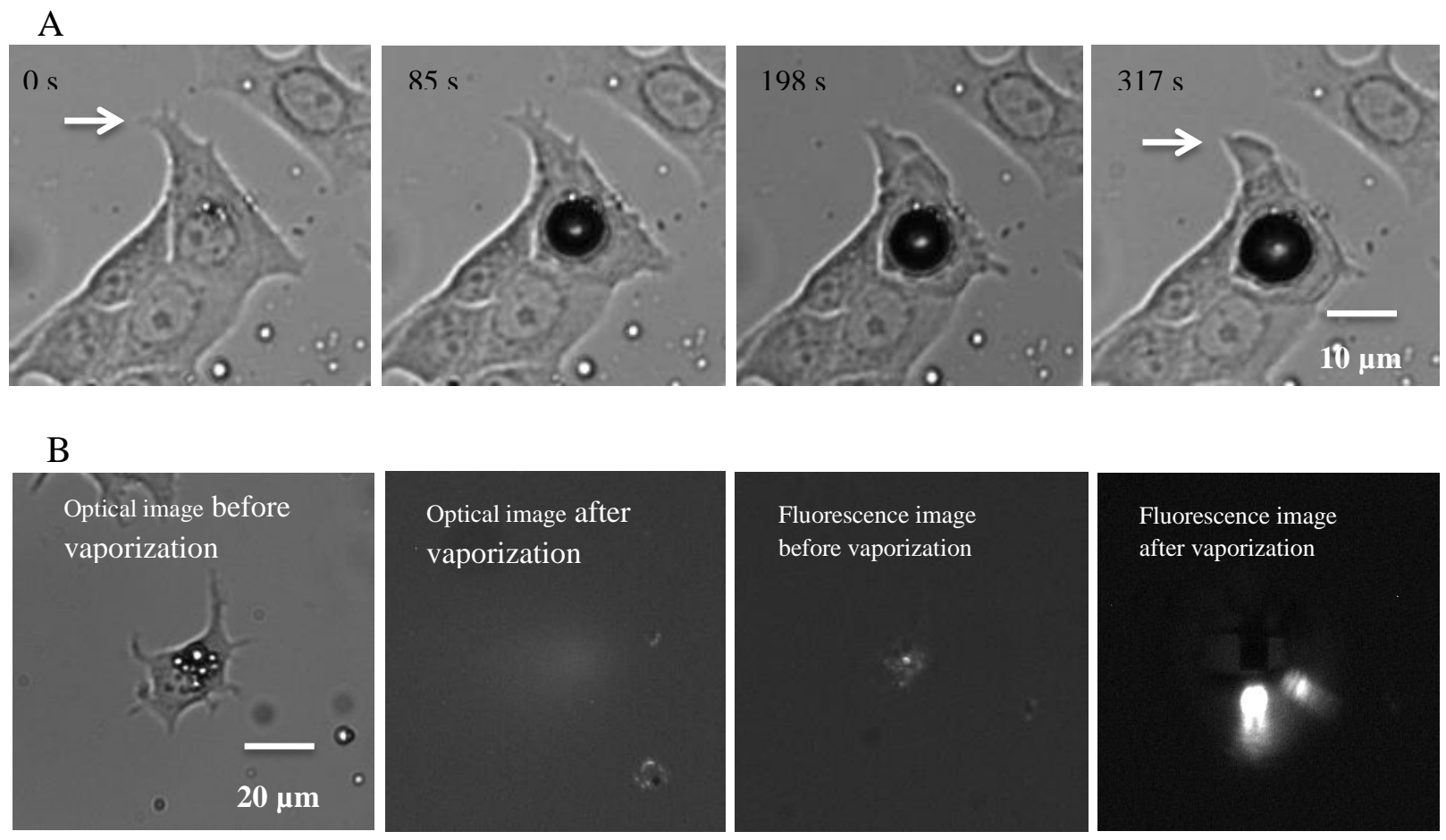

Fig. 3.8 (A) The MCF7 cell slowly retracted after vaporization (indicated by the white arrows). (B) Vaporization caused membrane disintegration evidenced by the PI fluorescence 20 seconds after laser irradiation. When the cell is not attached to other cells, it was lifted off the substrate by the bubble which could be seen in the real time microscope imaging.

This chapter's focus was to investigate the passive interactions that PLGA/PFH-GNPs have with cells. It was determined that PLGA/PFH-GNPs, in their final product stage, do not 
induce detectable cytotoxic affects in breast cancer cells. Furthermore, passive targeting and internalization of the PLGA/PFH-GNPs by cancer cells was achieved. Finally, the effect of localized PLGA/PFH-GNP vaporization inside the cells was studied. It was shown that vaporization impacts cell viability. Overall, PLGA/PFH-GNPs show promise of usage in biological settings, with minimal safety concern to the healthy cells prior to any laser triggered event. They also have potential as therapeutic agents for cancer therapy. 


\section{Chapter 4 - Conclusion and Future Work}

\subsection{Motivation}

Cancer remains one of the most deadly diseases in the world, and the number of new cases increases each year [144][145]. New methods for detection and treatment have dramatically improved cancer care. However, improved detection and increasing exposure to carcinogens has led to higher rates of cancer incidence. Therefore, the overall survival rate from cancer has not improved substantially over the past 30 years [146]. There is a vital need for agents that can be used for simultaneous detection, diagnosis and remotely triggered therapy for selective destruction of diseases tissue.

The main goal of this work was to design, synthesize, characterize, and test a nano- and micro-scale, dual contrast agent that worked simultaneously for combined ultrasound and photoacoustic imaging. Beyond its contrast enhancing abilities, the agent therapeutic applications were explored. Overall, this agent has the potential to be used for a multitude of purposes and applications due to their flexibility and adaptability for diagnostic and therapeutic applications.

\subsection{Phase Change Contrast Agent Synthesis and Characterization}

PLGA/PFH-GNPs were synthesized via a "water-oil-water" double emulsion technique that produces naturally PLGA shelled PFC droplets in which specially capped gold nanoparticles are suspended. Analysis and characterization through UV-visible spectrophotometry, TEM, SEM, dynamic light scattering, and AAS verify that PLGA/PFH-GNPs can be synthesized with highly reproducible properties. The PA properties of PLGA/PFH-GNPs were characterized using an 
acoustic microscope with an ultra-high frequency transducer. The vaporization thresholds were also determined according to the PLGA particle and GNP sizes. Overall, the synthesis method provided a simple way to create a dual contrast agent for combined PA and US imaging that is highly adaptable to specific applications in terms of particle size, the number of nanoparticles encapsulated, and the addition of surface coatings. In addition, knowing the contrast physical and PA properties will help us to optimize the experiment parameters for the future study.

\subsection{Cellular Interactions and Cytotoxicity}

The studies completed in this thesis show that PLGA/PFH-GNPs have no cytotoxic effects after simple incubation with cells for 24 hours. This is not surprising, considering that PLGA/PFH-GNPs use materials that are FDA approved for use with other materials that are currently undergoing rigorous cytotoxicity trials (GNPs). The silica coated or fluorinated GNPs have had the majority of the initial synthesis ligand. There is some question about the toxicity of organically coated GNPs expelled from the droplets. These question could be investigated using further animal studies, but current data suggest that gold is simply retained in the liver and spleen or excreted in the feces with limited, if any, long term effects [139]. While these studies can only be broadly generalized with regards to the clinical application of PLGA/PFH-GNPs, initial studies suggest limited chemical cytotoxicity.

\subsection{Contrast Enhancing Abilities of PLGA/PFH-GNPs}

The phantom and in vitro studies performed showed the strong PA signal produce with minimal light energy which indicates the potential of the PLGA/PFH-GNPs as PA contrast agent in the clinical application for shallow tissue image. If the optical absorbers are replaced by other 
types such as gold nanorods which absorb near infrared light, these agents' primary uses could be for relatively deep tissue imaging. Imaging with laser wavelengths at $1064 \mathrm{~nm}$ minimize background signal from tissue which further enhances the image contrast. A few of these applications include breast cancer, pancreatic cancer, and prostate cancer imaging. These organs are too deep to image using traditional optical modalities, as light delivery is prevented by the high tissue absorption and scattering.

\subsection{Future Work}

In this work, PLGA/PFH-GNP particles containing silica-coated gold nanoparticles were developed as phase-change contrast agents for photoacoustic imaging. In their liquid state, these agents generate strong photoacoustic signals which can be controlled by adjusting the laser energy and the particle physical properties. The particle vaporization via laser irradiation results in mechanical damage to cells. The methods used for imaging are non-destructive and convert light to acoustic transients with high efficiency.

Further investigation can be focused on several aspects including: 1. Using optical absorbers such as gold nanorods which can absorb NIR light to help increase image depth; 2. Making nanosized contrast agents $(<200 \mathrm{~nm})$ to improve the accumulation efficiency on the tumor site; 3. Modify the particle surface with molecular ligands to assist specific cell targeting; 4. Encapsulate therapeutics to further improve treatment efficiency. Overall, the continued work will be focused on optimizing these contrast agents which would image deeper tissue with more sensitivity and specificity than existing techniques, and offer improved outcomes for disease diagnostics and treatment. 


\section{References}

[1] J. T. B. PhD, J. A. Seibert, E. M. Leidholdt, and J. M. Boone, The Essential Physics of Medical Imaging, 2 edition. Philadelphia: Lippincott Williams \& Wilkins, 2001.

[2] J. L. Prince and J. Links, Medical Imaging Signals and Systems, 1 edition. Upper Saddle River, NJ: Prentice Hall, 2005.

[3] G. Rahbar, A. C. Sie, G. C. Hansen, J. S. Prince, M. L. Melany, H. E. Reynolds, V. P. Jackson, J. W. Sayre, and L. W. Bassett, "Benign versus Malignant Solid Breast Masses: US Differentiation," Radiology, vol. 213, no. 3, pp. 889-894, 1999.

[4] J. P. Culver, R. Choe, M. J. Holboke, L. Zubkov, T. Durduran, A. Slemp, V. Ntziachristos, B. Chance, and A. G. Yodh, "Three-dimensional diffuse optical tomography in the parallel plane transmission geometry: Evaluation of a hybrid frequency domain/continuous wave clinical system for breast imaging," Med. Phys., vol. 30, no. 2, p. 235, 2003.

[5] A. Rosencwaig, Photoacoustics and Photoacoustic Spectroscopy. New York: John Wiley \& Sons Inc, 1981.

[6] M. Friebel, K. Do, A. Hahn, G. Mu, and others, "Optical properties of circulating human blood in the wavelength range 400-2500 nm," J. Biomed. Opt., vol. 4, no. 1, pp. 36-46, 1999.

[7] S. Szabo and Z. Sandor, "The diagnostic and prognostic value of tumor angiogenesis," Eur. J. Surg., vol. 164, no. S12, pp. 99-103, 1998.

[8] P. Carmeliet and R. K. Jain, “Angiogenesis in cancer and other diseases," Nature, vol. 407, no. 6801, pp. 249-257, 2000.

[9] R. E. Apfel, “Activatable infusable dispersions containing drops of a superheated liquid for methods of therapy and diagnosis," US5840276 A, 24-Nov-1998.

[10] O. D. Kripfgans, J. B. Fowlkes, D. L. Miller, O. P. Eldevik, and P. L. Carson, "Acoustic droplet vaporization for therapeutic and diagnostic applications," Ultrasound Med. Biol., vol. 26, no. 7, pp. 1177-1189, 2000.

[11] E. M. Strohm, I. Gorelikov, N. Matsuura, and M. C. Kolios, "Acoustic and photoacoustic characterization of micron-sized perfluorocarbon emulsions," J. Biomed. Opt., vol. 17, no. 9, pp. 0960161-0960169, 2012.

[12] J.-Y. Fang, C.-F. Hung, M.-H. Liao, and C.-C. Chien, "A study of the formulation design of acoustically active lipospheres as carriers for drug delivery," Eur. J. Pharm. Biopharm., vol. 67, no. 1, pp. 67-75, 2007. 
[13] K. Wilson, K. Homan, and S. Emelianov, "Biomedical photoacoustics beyond thermal expansion using triggered nanodroplet vaporization for contrast-enhanced imaging," Nat. Commun., vol. 3, p. 618, 2012.

[14] Y. Sun, C. Niu, Y. Zheng, H. Ran, Z. Wang, Y. J. Wang, E. M. Strohm, and M. C. Kolios, "Vaporization, photoacoustic and acoustic characterization of PLGA/PFH particles loaded with optically absorbing materials," in Ultrasonics Symposium (IUS), IEEE International, 2013, pp. 132-135.

[15] E. Strohm, M. Rui, I. Gorelikov, N. Matsuura, and M. Kolios, "Vaporization of perfluorocarbon droplets using optical irradiation," Biomed. Opt. Express, vol. 2, no. 6, pp. 1432-1442, 2011.

[16] D. Christensen, Ultrasonic Bioinstrumentation. New York: Wiley, 1988.

[17] P. R. Hoskins, K. Martin, and A. Thrush, Eds., Diagnostic Ultrasound, 2 edition. Cambridge University Press, 2013.

[18] G. B. ALEXANDER, "On the production and reproduction of sound by light," Am. J. Sci., vol. 20, p. 305, 1880.

[19] A. Karabutov, N. B. Podymova, and V. S. Letokhov, "Time-resolved laser optoacoustic tomography of inhomogeneous media," Appl. Phys. B, vol. 63, no. 6, pp. 545-563, 1996.

[20] C. G. A. Hoelen, F. F. M. De Mul, R. Pongers, and A. Dekker, "Three-dimensional photoacoustic imaging of blood vessels in tissue," Opt. Lett., vol. 23, no. 8, pp. 648-650, 1998.

[21] L. V. Wang, X. Zhao, H. Sun, and G. Ku, "Microwave-induced acoustic imaging of biological tissues," Rev. Sci. Instrum., vol. 70, no. 9, pp. 3744-3748, 1999.

[22] X. Wang, Y. Pang, G. Ku, G. Stoica, and L. V. Wang, "Three-dimensional laser-induced photoacoustic tomography of mouse brain with the skin and skull intact," Opt. Lett., vol. 28, no. 19, pp. 1739-1741, 2003.

[23] A. A. Oraevsky, S. L. Jacques, and F. K. Tittel, "Measurement of tissue optical properties by time-resolved detection of laser-induced transient stress," Appl. Opt., vol. 36, no. 1, pp. 402$415,1997$.

[24] R. A. Kruger, "Photoacoustic ultrasound," Med. Phys., vol. 21, no. 1, p. 127, 1994.

[25] M. Xu, "Photoacoustic imaging in biomedicine," vol. 77, no. 4, p. 041101, 2006.

[26] A. A. Oraevsky, E. V. Savateeva, S. V. Solomatin, A. A. Karabutov, V. G. Andreev, Z. Gatalica, T. Khamapirad, and P. M. Henrichs, "Optoacoustic imaging of blood for visualization and diagnostics of breast cancer," 2002, vol. 4618, pp. 81-94.

[27] L. V. Wang, "Tutorial on Photoacoustic Microscopy and Computed Tomography," IEEE J. Sel. Top. Quantum Electron., vol. 14, no. 1, pp. 171-179, 2008. 
[28] L. M. Lyamshev, "Optoacoustic sources of sound," Phys.-Uspekhi, vol. 24, no. 12, pp. 977995, 1981.

[29] K. E. Wilson, "Biomedical photoacoustics beyond thermal expansion : photoacoustic nanoDroplets," University of Texas, Austin, 2012.

[30] Anonymous, "Laser safety standard," Manuf. Eng., vol. 125, no. 4, p. 22, 2000.

[31] A. A. Oraevsky, S. L. Jacques, and F. K. Tittel, "Determination of tissue optical properties by piezoelectric detection of laser-induced stress waves," in OE/LASE'93: Optics, ElectroOptics, \& Laser Applications in Science \& Engineering, 1993, pp. 86-101.

[32] Karabutov, Laser Optoacoustics, 1st edition. New York: Springer/Sci-Tech/Trade, 1993.

[33] X. Yang, E. W. Stein, S. Ashkenazi, and L. V. Wang, "Nanoparticles for photoacoustic imaging," Wiley Interdiscip. Rev. Nanomed. Nanobiotechnol., vol. 1, no. 4, pp. 360-368, 2009.

[34] M. A. Phillips, M. L. Gran, and N. A. Peppas, "Targeted nanodelivery of drugs and diagnostics," Nano Today, vol. 5, no. 2, pp. 143-159, 2010.

[35] C. Zhang, Y. S. Zhang, D.-K. Yao, Y. Xia, and L. V. Wang, "Label-free photoacoustic microscopy of cytochromes," J. Biomed. Opt., vol. 18, no. 2, pp. 020504-020504, 2013.

[36] Y. Zhou, "Photoacoustic microscopy of bilirubin in tissue phantoms," J. Biomed. Opt., vol. 17, no. 12, p. 126019, 2012.

[37] C. Zhang, Y.-J. Cheng, J. Chen, S. Wickline, and L. V. Wang, "Label-free photoacoustic microscopy of myocardial sheet architecture," J. Biomed. Opt., vol. 17, no. 6, pp. 06050610605063, 2012.

[38] J. Staley, P. Grogan, A. K. Samadi, H. Cui, M. S. Cohen, and X. Yang, "Growth of melanoma brain tumors monitored by photoacoustic microscopy," J. Biomed. Opt., vol. 15, no. 4, p. 040510, 2010.

[39] C. Zhang, K. Maslov, and L. V. Wang, "Subwavelength-resolution label-free photoacoustic microscopy of optical absorption in vivo," Opt. Lett., vol. 35, no. 19, pp. 3195-3197, 2010.

[40] V. V. Yakovlev, H. F. Zhang, G. D. Noojin, M. L. Denton, R. J. Thomas, and M. O. Scully, "Stimulated Raman photoacoustic imaging," Proc. Natl. Acad. Sci., vol. 107, no. 47, pp. 20335-20339, 2010.

[41] Z. Xu, C. Li, and L. V. Wang, "Photoacoustic tomography of water in phantoms and tissue," J. Biomed. Opt., vol. 15, no. 3, p. 036019, 2010.

[42] H. A. MacKenzie, H. S. Ashton, S. Spiers, Y. Shen, S. S. Freeborn, J. Hannigan, J. Lindberg, and P. Rae, "Advances in photoacoustic noninvasive glucose testing," Clin. Chem., vol. 45, no. 9 , pp. $1587-1595,1999$. 
[43] J. Yao and L. V. Wang, "Sensitivity of photoacoustic microscopy," Photoacoustics, vol. 2, no. 2, pp. 87-101, 2014.

[44] J. Yao and L. V. Wang, "Photoacoustic microscopy,” Laser Photon Rev, pp. 758-78, 2013.

[45] G. P. Luke, D. Yeager, and S. Y. Emelianov, "Biomedical Applications of Photoacoustic Imaging with Exogenous Contrast Agents," Ann. Biomed. Eng., vol. 40, no. 2, pp. 422-437, 2012.

[46] X. Yang, S. E. Skrabalak, Z.-Y. Li, Y. Xia, and L. V. Wang, "Photoacoustic Tomography of a Rat Cerebral Cortex in vivo with Au Nanocages as an Optical Contrast Agent," Nano Lett., vol. 7, no. 12, pp. 3798-3802, 2007.

[47] G. Ku and L. V. Wang, "Deeply penetrating photoacoustic tomography in biological tissues enhanced with an optical contrast agent," Opt. Lett., vol. 30, no. 5, pp. 507-509, 2005.

[48] J. Yao, K. Maslov, S. Hu, and L. V. Wang, "Evans blue dye-enhanced capillary-resolution photoacoustic microscopy in vivo," J. Biomed. Opt., vol. 14, no. 5, p. 054049, 2009.

[49] C. Kim, E. C. Cho, J. Chen, K. H. Song, L. Au, C. Favazza, Q. Zhang, C. M. Cobley, F. Gao, Y. Xia, and L. V. Wang, "Molecular Photoacoustic Tomography of Melanomas Targeted by Bioconjugated Gold Nanocages," ACS Nano, vol. 4, no. 8, pp. 4559-4564, 2010.

[50] E. I. Galanzha, E. V. Shashkov, T. Kelly, J.-W. Kim, L. Yang, and V. P. Zharov, "In vivo magnetic enrichment and multiplex photoacoustic detection of circulating tumour cells," Nat. Nanotechnol., vol. 4, no. 12, pp. 855-860, 2009.

[51] J. Copland, M. Eghtedari, V. Popov, N. Kotov, N. Mamedova, M. Motamedi, and A. Oraevsky, "Bioconjugated gold nanoparticles as a molecular based contrast agent: implications for imaging of deep tumors using optoacoustic tomography," Mol. Imaging Biol., vol. 6, no. 5, pp. 341-349, 2004.

[52] D. Pan, M. Pramanik, A. Senpan, S. Ghosh, S. A. Wickline, L. V. Wang, and G. M. Lanza, "Near infrared photoacoustic detection of sentinel lymph nodes with gold nanobeacons," Biomaterials, vol. 31, no. 14, pp. 4088-4093, 2010.

[53] K. H. Song, C. Kim, C. M. Cobley, Y. Xia, and L. V. Wang, "Near-Infrared Gold Nanocages as a New Class of Tracers for Photoacoustic Sentinel Lymph Node Mapping on a Rat Model," Nano Lett., vol. 9, no. 1, pp. 183-188, 2009.

[54] D. Pan, X. Cai, C. Yalaz, A. Senpan, K. Omanakuttan, S. A. Wickline, L. V. Wang, and G. M. Lanza, "Photoacoustic Sentinel Lymph Node Imaging with Self-Assembled Copper Neodecanoate Nanoparticles," ACS Nano, vol. 6, no. 2, pp. 1260-1267, 2012.

[55] C. M. Pitsillides, E. K. Joe, X. Wei, R. Anderson, and C. P. Lin, "Selective cell targeting with light-absorbing microparticles and nanoparticles," Biophys. J., vol. 84, no. 6, pp. 40234032, 2003. 
[56] A. M. Gobin, M. H. Lee, N. J. Halas, W. D. James, R. A. Drezek, and J. L. West, "NearInfrared Resonant Nanoshells for Combined Optical Imaging and Photothermal Cancer Therapy," Nano Lett., vol. 7, no. 7, pp. 1929-1934, 2007.

[57] P. P. Joshi, Y.-S. Chen, S. Kim, J. Shah, K. Sokolov, and S. Emelianov, "Molecular therapeutic agents for noninvasive photoacoustic image-guided photothermal therapy," in Engineering in Medicine and Biology Society, EMBC, Annual International Conference of the IEEE, 2009, pp. 4106-4109.

[58] S. K. Ghosh and T. Pal, "Interparticle Coupling Effect on the Surface Plasmon Resonance of Gold Nanoparticles: From Theory to Applications," Chem. Rev., vol. 107, no. 11, pp. 47974862, 2007.

[59] S. Mallidi, T. Larson, J. Aaron, K. Sokolov, and S. Emelianov, "Molecular specific optoacoustic imaging with plasmonic nanoparticles," Opt. Express, vol. 15, no. 11, pp. 6583-6588, 2007.

[60] A. A. Oraevsky, A. A. Karabutov, and E. V. Savateeva, "Enhancement of optoacoustic tissue contrast with absorbing nanoparticles," in European Conference on Biomedical Optics, 2001, pp. 60-69.

[61] W. Lu, Q. Huang, G. Ku, X. Wen, M. Zhou, D. Guzatov, P. Brecht, R. Su, A. Oraevsky, L. V. Wang, and C. Li, "Photoacoustic imaging of living mouse brain vasculature using hollow gold nanospheres," Biomaterials, vol. 31, no. 9, pp. 2617-2626, 2010.

[62] Y. Wang, X. Xie, X. Wang, G. Ku, K. L. Gill, D. P. O’Neal, G. Stoica, and L. V. Wang, "Photoacoustic Tomography of a Nanoshell Contrast Agent in the in Vivo Rat Brain," Nano Lett., vol. 4, no. 9, pp. 1689-1692, 2004.

[63] M. Eghtedari, A. Oraevsky, J. A. Copland, N. A. Kotov, A. Conjusteau, and M. Motamedi, "High Sensitivity of In Vivo Detection of Gold Nanorods Using a Laser Optoacoustic Imaging System," Nano Lett., vol. 7, no. 7, pp. 1914-1918, 2007.

[64] P.-C. Li, C.-W. Wei, C.-K. Liao, C.-D. Chen, K.-C. Pao, C.-R. C. Wang, Y.-N. Wu, and D.B. Shieh, "Photoacoustic Imaging of Multiple Targets Using Gold Nanorods," IEEE Trans. Ultrason. Ferroelectr. Freq. Control, vol. 54, no. 8, pp. 1642-1647, 2007.

[65] K. Homan, J. Shah, S. Gomez, H. Gensler, A. Karpiouk, L. Brannon-Peppas, and S. Emelianov, "Combined ultrasound and photoacoustic imaging of pancreatic cancer using nanocage contrast agents," in Proc. of SPIE, 2009, vol. 71771M-71771M-6.

[66] K. Homan, S. Kim, Y.-S. Chen, B. Wang, S. Mallidi, and S. Emelianov, "Prospects of molecular photoacoustic imaging at 1064 nm wavelength," Opt. Lett., vol. 35, no. 15, pp. 2663-2665, 2010.

[67] O. D. Kripfgans, J. B. Fowlkes, D. L. Miller, O. P. Eldevik, and P. L. Carson, "Acoustic droplet vaporization for therapeutic and diagnostic applications," Ultrasound Med. Biol., vol. 26, no. 7, pp. 1177-1189, 2000. 
[68] M. Zhang, M. L. Fabiilli, K. J. Haworth, J. B. Fowlkes, O. D. Kripfgans, W. W. Roberts, K. A. Ives, and P. L. Carson, "Initial Investigation of Acoustic Droplet Vaporization for Occlusion in Canine Kidney," Ultrasound Med. Biol., vol. 36, no. 10, pp. 1691-1703, 2010.

[69] N. Reznik, R. Williams, and P. N. Burns, "Investigation of Vaporized Submicron Perfluorocarbon Droplets as an Ultrasound Contrast Agent," Ultrasound Med. Biol., vol. 37 , no. 8, pp. 1271-1279, 2011.

[70] N. Matsuura, R. Williams, I. Gorelikov, J. Chaudhuri, J. Rowlands, K. Hynynen, S. Foster, P. Burns, and N. Resnik, "Nanoparticle-loaded perfluorocarbon droplets for imaging and therapy,” in Ultrasonics Symposium (IUS), IEEE International, 2009, pp. 5-8.

[71] P. Zhang and T. Porter, "An in vitro study of a phase-shift nanoemulsion: a potential nucleation agent for bubble-enhanced HIFU tumor ablation," Ultrasound Med. Biol., vol. 36, no. 11, pp. 1856-1866, 2010.

[72] M. L. Fabiilli, J. A. Lee, O. D. Kripfgans, P. L. Carson, and J. B. Fowlkes, "Delivery of Water-Soluble Drugs Using Acoustically Triggered Perfluorocarbon Double Emulsions,' Pharm. Res., vol. 27, no. 12, pp. 2753-2765, 2010.

[73] N. Y. Rapoport, K.-H. Nam, Z. Gao, and A. Kennedy, "Application of ultrasound for targeted nanotherapy of malignant tumors," Acoust. Phys., vol. 55, no. 4-5, pp. 594-601, 2009.

[74] O. D. Kripfgans, J. B. Fowlkes, M. Woydt, O. P. Eldevik, and P. L. Carson, "In vivo droplet vaporization for occlusion therapy and phase aberration correction," Ultrason. Ferroelectr. Freq. Control IEEE Trans. On, vol. 49, no. 6, pp. 726-738, 2002.

[75] M. L. Fabiilli, I. E. Sebastian, K. J. Haworth, O. D. Kripfgans, P. L. Carson, and J. B. Fowlkes, "Ultrasonic delivery of a chemotherapeutic agent using acoustic droplet vaporization (ADV),” in Ultrasonics Symposium (IUS), 2009 IEEE International, 2009, pp. 101-103.

[76] P. S. Sheeran, S. Luois, P. A. Dayton, and T. O. Matsunaga, "Formulation and Acoustic Studies of a New Phase-Shift Agent for Diagnostic and Therapeutic Ultrasound," Langmuir, vol. 27, no. 17, pp. 10412-10420, 2011.

[77] E. M. Strohm, M. Rui, M. C. Kolios, I. Gorelikov, and N. Matsuura, "Optical droplet vaporization (ODV): photoacoustic characterization of perfluorocarbon droplets," in Ultrasonics Symposium (IUS), IEEE, 2010, pp. 495-498.

[78] Y. Sun, Y. Wang, C. Niu, E. M. Strohm, Y. Zheng, H. Ran, R. Huang, D. Zhou, Y. Gong, Z. Wang, D. Wang, and M. C. Kolios, "Laser-Activatible PLGA Microparticles for ImageGuided Cancer Therapy In Vivo," Adv. Funct. Mater., Sep. 2014.

http://onlinelibrary.wiley.com/doi/10.1002/adfm.201402631/abstract 
[79] S. K. Ghosh and T. Pal, "Interparticle Coupling Effect on the Surface Plasmon Resonance of Gold Nanoparticles: From Theory to Applications," Chem. Rev., vol. 107, no. 11, pp. 47974862, 2007.

[80] N. Matsuura, R. Williams, I. Gorelikov, J. Chaudhuri, J. Rowlands, K. Hynynen, S. Foster, P. Burns, and N. Resnik, "Nanoparticle-loaded perfluorocarbon droplets for imaging and therapy,” in Ultrasonics Symposium (IUS), IEEE International, 2009, pp. 5-8.

[81] J.-Y. Fang, C.-F. Hung, M.-H. Liao, and C.-C. Chien, "A study of the formulation design of acoustically active lipospheres as carriers for drug delivery," Eur. J. Pharm. Biopharm., vol. 67, no. 1, pp. 67-75, 2007.

[82] Y.-S. Chen, W. Frey, S. Kim, P. Kruizinga, K. Homan, and S. Emelianov, "Silica-Coated Gold Nanorods as Photoacoustic Signal Nanoamplifiers," Nano Lett., vol. 11, no. 2, pp. 348354, 2011.

[83] R. F. Mattrey, "The potential role of perfluorochemicals (PFCs) in diagnostic imaging," Artif. Cells Blood Substit. Biotechnol., vol. 22, no. 2, pp. 295-313, 1994.

[84] M. P. Krafft, "Fluorocarbons and fluorinated amphiphiles in drug delivery and biomedical research," Adv. Drug Deliv. Rev., vol. 47, no. 2, pp. 209-228, 2001.

[85] W. Cui, J. Bei, S. Wang, G. Zhi, Y. Zhao, X. Zhou, H. Zhang, and Y. Xu, "Preparation and evaluation of poly(L-lactide-co-glycolide) (PLGA) microbubbles as a contrast agent for myocardial contrast echocardiography," J. Biomed. Mater. Res. B Appl. Biomater., vol. 73, no. 1, pp. 171-178, 2005.

[86] E. Pisani, N. Tsapis, J. Paris, V. Nicolas, L. Cattel, and E. Fattal, "Polymeric Nano/Microcapsules of Liquid Perfluorocarbons for Ultrasonic Imaging: Physical Characterization," Langmuir, vol. 22, no. 9, pp. 4397-4402, 2006.

[87] K. E. Uhrich, S. M. Cannizzaro, R. S. Langer, and K. M. Shakesheff, "Polymeric Systems for Controlled Drug Release,” Chem. Rev., vol. 99, no. 11, pp. 3181-3198, 1999.

[88] F. Danhier, "PLGA-based nanoparticles: An overview of biomedical applications," J. Controlled Release, vol. 161, no. 2, pp. 505-522, 2012.

[89] M. W. Sigrist, "Laser generation of acoustic waves in liquids and gases," J. Appl. Phys., vol. 60, no. 7, p. R83, 1986.

[90] T. Asshauer, K. Rink, and G. Delacretaz, "Acoustic transient generation by holmium-laserinduced cavitation bubbles,” J. Appl. Phys., vol. 76, no. 9, pp. 5007-5013, 1994.

[91] L. D. Landau and E. M. Lifshitz, Fluid Mechanics: Volume 6, 2 edition. Oxford: Butterworth-Heinemann, 1987.

[92] Z. Zhao, S. Glod, and D. Poulikakos, "Pressure and power generation during explosive vaporization on a thin-film microheater," Int. J. Heat Mass Transf., vol. 43, no. 2, pp. 281296, 2000. 
[93] P. Rai, S. Mallidi, X. Zheng, R. Rahmanzadeh, Y. Mir, S. Elrington, A. Khurshid, and T. Hasan, "Development and applications of photo-triggered theranostic agents," Adv. Drug Deliv. Rev., vol. 62, no. 11, pp. 1094-1124, 2010.

[94] M. A. C. Stuart, W. T. S. Huck, J. Genzer, M. Müller, C. Ober, M. Stamm, G. B. Sukhorukov, I. Szleifer, V. V. Tsukruk, M. Urban, F. Winnik, S. Zauscher, I. Luzinov, and S. Minko, "Emerging applications of stimuli-responsive polymer materials," Nat. Mater., vol. 9, no. 2, pp. 101-113, 2010.

[95] L. K. Bogart, A. Taylor, Y. Cesbron, P. Murray, and R. Lévy, "Photothermal Microscopy of the Core of Dextran-Coated Iron Oxide Nanoparticles During Cell Uptake," ACS Nano, vol. 6, no. 7, pp. 5961-5971, 2012.

[96] F.-Y. Cheng, "Multifunctional polymeric nanoparticles for combined chemotherapeutic and near-infrared photothermal cancer therapy in vitro and in vivo," Chem. Commun., vol. 46, no. 18, pp. 3167-3169, 2010.

[97] J. U. Menon, P. Jadeja, P. Tambe, K. Vu, B. Yuan, and K. T. Nguyen, "Nanomaterials for Photo-Based Diagnostic and Therapeutic Applications," Theranostics, vol. 3, no. 3, pp. 152$166,2013$.

[98] L. Cheng, K. Yang, Y. Li, X. Zeng, M. Shao, S.-T. Lee, and Z. Liu, "Multifunctional nanoparticles for upconversion luminescence/MR multimodal imaging and magnetically targeted photothermal therapy," Biomaterials, vol. 33, no. 7, pp. 2215-2222, 2012.

[99] B. Khlebtsov, E. Panfilova, V. Khanadeev, O. Bibikova, G. Terentyuk, A. Ivanov, V. Rumyantseva, I. Shilov, A. Ryabova, V. Loshchenov, and N. G. Khlebtsov, "Nanocomposites Containing Silica-Coated Gold-Silver Nanocages and Yb-2,4Dimethoxyhematoporphyrin: Multifunctional Capability of IR-Luminescence Detection, Photosensitization, and Photothermolysis," ACS Nano, vol. 5, no. 9, pp. 7077-7089, 2011.

[100] S.-H. Cheng, C.-H. Lee, M.-C. Chen, J. S. Souris, F.-G. Tseng, C.-S. Yang, C.-Y. Mou, C.-T. Chen, and L.-W. Lo, "Tri-functionalization of mesoporous silica nanoparticles for comprehensive cancer theranostics - the trio of imaging, targeting and therapy," J. Mater. Chem., vol. 20, no. 29, p. 6149, 2010.

[101] E. C. Unger, "Therapeutic applications of lipid-coated microbubbles," Adv. Drug Deliv. Rev., vol. 56, no. 9, pp. 1291-1314, 2004.

[102] P. A. Dijkmans, L. J. M. Juffermans, R. J. P. Musters, A. van Wamel, F. J. ten Cate, W. van Gilst, C. A. Visser, N. de Jong, and O. Kamp, "Microbubbles and ultrasound: from diagnosis to therapy," Eur. Heart J. - Cardiovasc. Imaging, vol. 5, no. 4, pp. 245-246, 2004.

[103] P.G. de Gennes, F. Brochard-Wyart, and D. Quere, Capillarity and Wetting Phenomena: Drops, Bubbles, Pearls, Waves, 2004 edition. New York: Springer, 2003.

[104] M. J. Rosen and J. T. Kunjappu, Surfactants and Interfacial Phenomena, 4 edition. Wiley, 2012. 
[105] S. F. Flaim, "Pharmacokinetics and Side Effects of Perfluorocarbon-Based Blood Substitutes," Artif. Cells Blood Substit. Biotechnol., vol. 22, no. 4, pp. 1043-1054, 1994.

[106] Evan Unger and TerryO Matsunaga, "Lipid Coated Microbubbles And Nanodroplets As Tools For Biomedical Nanotechnology," in Handbook of Materials for Nanomedicine, 0 vols., Pan Stanford Publishing, 2010, pp. 749-786.

[107] M. A. Borden, D. E. Kruse, C. F. Caskey, S. Zhao, P. A. Dayton, and K. W. Ferrara, "Influence of lipid shell physicochemical properties on ultrasound-induced microbubble destruction," IEEE Trans. Ultrason. Ferroelectr. Freq. Control, vol. 52, no. 11, pp. 19922002, 2005.

[108] A. Michnik, "Thermal stability of bovine serum albumin DSC study," J. Therm. Anal. Calorim., vol. 71, no. 2, pp. 509-519. 2003.

[109] A. Michnik, "Stability of bovine serum albumin at different pH," J. Therm. Anal. Calorim., vol. 80, no. 2, pp. 399-406, 2005.

[110] J. S. Xu, "Synthesizing and binding dual-mode poly (lactic-co-glycolic acid) (PLGA) nanobubbles for cancer targeting and imaging," Biomaterials, vol. 31, no. 7, pp. 1716-1722, Mar. 2010.

[111] J. M. Anderson, "Biodegradation and biocompatibility of PLA and PLGA microspheres," Adv. Drug Deliv. Rev., vol. 28, no. 1, pp. 5-24, 1997.

[112] J. A. Straub, "Porous PLGA microparticles: AI-700, an intravenously administered ultrasound contrast agent for use in echocardiography," J. Controlled Release, vol. 108, no. 1, pp. 21-32, 2005.

[113] D. M. El-Sherif and M. A. Wheatley, "Development of a novel method for synthesis of a polymeric ultrasound contrast agent," J. Biomed. Mater. Res. A, vol. 66A, no. 2, pp. 347-355, 2003.

[114] A. A. Oraevsky, A. A. Karabutov, and E. V. Savateeva, "Enhancement of optoacoustic tissue contrast with absorbing nanoparticles," in European Conference on Biomedical Optics, 2001, pp. 60-69.

[115] A. Agarwal, S. W. Huang, M. O’Donnell, K. C. Day, M. Day, N. Kotov, and S. Ashkenazi, "Targeted gold nanorod contrast agent for prostate cancer detection by photoacoustic imaging," J. Appl. Phys., vol. 102, no. 6, p. 064701, 2007.

[116] M. Eghtedari, A. Oraevsky, J. A. Copland, N. A. Kotov, A. Conjusteau, and M. Motamedi, "High Sensitivity of In Vivo Detection of Gold Nanorods Using a Laser Optoacoustic Imaging System," Nano Lett., vol. 7, no. 7, pp. 1914-1918, 2007.

[117] K. E. Wilson, K. A. Homan, and S. Y. Emelianov, "Remotely triggered contrast nanoagent for ultrasound and photoacoustic imaging," in IEEE Ultrasonics Symposium (IUS), 2010, pp. 1003-1006. 
[118] K. Wilson, K. Homan, and S. Emelianov, "Synthesis of a dual contrast agent for ultrasound and photoacoustic imaging," in Proc. of SPIE, 2010, vol. 7576, p. 75760M-5.

[119] S. Abe and K. Kajikawa, "Linear and nonlinear optical properties of gold nanospheres immobilized on a metallic surface," Phys. Rev. B, vol. 74, no. 3, p. 035416, 2006.

[120] P. K. Jain, K. S. Lee, I. H. El-Sayed, and M. A. El-Sayed, "Calculated Absorption and Scattering Properties of Gold Nanoparticles of Different Size, Shape, and Composition: Applications in Biological Imaging and Biomedicine," J. Phys. Chem. B, vol. 110, no. 14, pp. 7238-7248, 2006.

[121] R. Elghanian, J. J. Storhoff, R. C. Mucic, R. L. Letsinger, and C. A. Mirkin, "Selective Colorimetric Detection of Polynucleotides Based on the Distance-Dependent Optical Properties of Gold Nanoparticles," Science, vol. 277, no. 5329, pp. 1078-1081, 1997.

[122] W. Lu, Q. Huang, G. Ku, X. Wen, M. Zhou, D. Guzatov, P. Brecht, R. Su, A. Oraevsky, L. V. Wang, and C. Li, "Photoacoustic imaging of living mouse brain vasculature using hollow gold nanospheres," Biomaterials, vol. 31, no. 9, pp. 2617-2626, 2010.

[123] C. J. Murphy, A. M. Gole, S. E. Hunyadi, J. W. Stone, P. N. Sisco, A. Alkilany, B. E. Kinard, and P. Hankins, "Chemical sensing and imaging with metallic nanorods," Chem. Commun., no. 5, pp. 544-557, 2008.

[124] Y.-S. Chen, W. Frey, S. Kim, K. Homan, P. Kruizinga, K. Sokolov, and S. Emelianov, "Enhanced thermal stability of silica-coated gold nanorods for photoacoustic imaging and image-guided therapy," Opt. Express, vol. 18, no. 9, pp. 8867-8878, 2010.

[125] B. Nikoobakht and M. A. El-Sayed, "Preparation and Growth Mechanism of Gold Nanorods (NRs) Using Seed-Mediated Growth Method," Chem. Mater., vol. 15, no. 10, pp. 1957-1962, 2003.

[126] X. Huang, "Gold Nanorods: From Synthesis and Properties to Biological and Biomedical Applications," Adv. Mater., vol. 21, no. 48, pp. 4880-4910, 2009.

[127] K. Homan, J. Shah, S. Gomez, H. Gensler, A. Karpiouk, L. Brannon-Peppas, and S. Emelianov, "Combined ultrasound and photoacoustic imaging of pancreatic cancer using nanocage contrast agents," 2009, vol. 7177, p. 71771M-71771M-6.

[128] S. L. Lombardi, Ed., Nanoparticles: new research. New York: Nova Science Publishers, 2008.

[129] J. Turkevich, P. C. Stevenson, and J. Hillier, "A study of the nucleation and growth processes in the synthesis of colloidal gold," Discuss. Faraday Soc., vol. 11, no. 0, pp. 55-75, 1951.

[130] G. Frens, "Particle size and sol stability in metal colloids," Kolloid-Z. Z. Für Polym., vol. 250, no. 7, pp. 736-741, 1972. 
[131] N. G. Bastús, J. Comenge, and V. Puntes, "Kinetically Controlled Seeded Growth Synthesis of Citrate-Stabilized Gold Nanoparticles of up to $200 \mathrm{~nm}$ : Size Focusing versus Ostwald Ripening," Langmuir, vol. 27, no. 17, pp. 11098-11105, 2011.

[132] S. H. Liu and M. Y. Han, "Synthesis, Functionalization, and Bioconjugation of Monodisperse, Silica-Coated Gold Nanoparticles: Robust Bioprobes," Adv. Funct. Mater., vol. 15 , no. 6, pp. 961-967, 2005.

[133] I. Gorelikov, A. L. Martin, M. Seo, and N. Matsuura, "Silica-Coated Quantum Dots for Optical Evaluation of Perfluorocarbon Droplet Interactions with Cells," Langmuir, vol. 27, no. 24, pp. 15024-15033, 2011.

[134] Y. J. Wang, E. M. Strohm, Y. Sun, C. Niu, Y. Zheng, Z. Wang, and M. C. Kolios, "PLGA/PFC particles loaded with gold nanoparticles as dual contrast agents for photoacoustic and ultrasound imaging," in Proc. of SPIE, 2014, vol. 8943, p. 89433M89433M-7.

[135] Y.-S. Chen, W. Frey, S. Aglyamov, and S. Emelianov, "Environment-Dependent Generation of Photoacoustic Waves from Plasmonic Nanoparticles," Small, vol. 8, no. 1, pp. 47-52, 2012.

[136] C. L. Didychuk, P. Ephrat, A. Chamson-Reig, S. L. Jacques, and J. J. L. Carson, "Depth of photothermal conversion of gold nanorods embedded in a tissue-like phantom," Nanotechnology, vol. 20, no. 19, p. 195102, 2009.

[137] R. Díaz-López, N. Tsapis, and E. Fattal, "Liquid Perfluorocarbons as Contrast Agents for Ultrasonography and 19F-MRI," Pharm. Res., vol. 27, no. 1, pp. 1-16, 2010.

[138] J. M. Correas and S. D. Quay, "EchoGen emulsion: a new ultrasound contrast agent based on phase shift colloids," Clin. Radiol., vol. 51 Suppl 1, pp. 11-14, 1996.

[139] N. Lewinski, V. Colvin, and R. Drezek, "Cytotoxicity of nanoparticles," Small Weinh. Bergstr. Ger., vol. 4, no. 1, pp. 26-49, 2008.

[140] I. Singh, Textbook of Human Histology: With Colour Atlas and Practical Guide, 6 edition. New Delhi ; St. Louis: Jp Medical Publishers, 2011.

[141] R. Cailleau, M. Olivé, and Q. V. J. Cruciger, "Long-term human breast carcinoma cell lines of metastatic origin: Preliminary characterization," In Vitro, vol. 14, no. 11, pp. 911-915, 1978.

[142] S. Cleator, W. Heller, and R. C. Coombes, "Triple-negative breast cancer: therapeutic options," Lancet Oncol., vol. 8, no. 3, pp. 235-244, 2007.

[143] T. O. Nielsen, F. D. Hsu, K. Jensen, M. Cheang, G. Karaca, Z. Hu, T. Hernandez-Boussard, C. Livasy, D. Cowan, L. Dressler, L. A. Akslen, J. Ragaz, A. M. Gown, C. B. Gilks, M. van de Rijn, and C. M. Perou, "Immunohistochemical and clinical characterization of the basallike subtype of invasive breast carcinoma," Clin. Cancer Res. Off. J. Am. Assoc. Cancer Res., vol. 10, no. 16, pp. 5367-5374, 2004. 
[144] P. Boyle and B. Levin, World Cancer Report 2008, 1 edition. Lyon: Geneva: WHO Publications, 2009.

[145] I. Agency, World Cancer Report 2014, 1 edition. Lyon, France: WHO Publications, 2014.

[146] “Canadian Cancer Statistics publication,” www.cancer.ca. [Online]. Available: http://www.cancer.ca/en/cancer-information/cancer-101/canadian-cancer-statisticspublication/?region=on. [Accessed: 06-Nov-2014]. 